\title{
Venus Atmospheric Thermal Structure and Radiative Balance
}

\author{
Sanjay S. Limaye ${ }^{1}$ Davide Grassi ${ }^{2}$ Arnaud Mahieux ${ }^{3}$. \\ Alessandra Migliorini ${ }^{2}$ Silvia Tellmann ${ }^{4}$. \\ Dmitrij Titov ${ }^{5}$
}

Received: 7 September 2017 / Accepted: 7 July 2018 / Published online: 13 August 2018

(C) The Author(s) 2018

\begin{abstract}
From the discovery that Venus has an atmosphere during the 1761 transit by M. Lomonosov to the current exploration of the planet by the Akatsuki orbiter, we continue to learn about the planet's extreme climate and weather. This chapter attempts to provide a comprehensive but by no means exhaustive review of the results of the atmospheric thermal structure and radiative balance since the earlier works published in Venus and Venus II books from recent spacecraft and Earth based investigations and summarizes the gaps in
\end{abstract}

Venus III

Edited by Bruno Bézard, Christopher T. Russell, Takehiko Satoh, Suzanne E. Smrekar and Colin F. Wilson

$凶$ S.S. Limaye

sslimaye@wisc.edu

D. Grassi

davide.grassi@iaps.inaf.it

A. Mahieux

arnaud.mahieux@aeronomie.be

A. Migliorini

alessandra.migliorini@iaps.inaf.it

S. Tellmann

stellman@uni-koeln.de

D. Titov

dmitri.titov@esa.int

1 Space Science and Engineering Center, University of Wisconsin, Madison, WI 53726, USA

2 IAPS - Institute for Space Astrophysics and Planetology, INAF - National Institute for Astrophysics, 00133 Rome, Italy

3 Institut d'Aéronomie Spatiale de Belgique (IASB), Belgische Instituut voor Ruimte en Aéronomie (BIRA), Belgian Institute for Space Aeronomy (BISA), 3, avenue Circulaire/Ringlaan 3,

1180 Brussel, Belgium

4 Department of Planetary Research, Rheinisches Institut für Umweltforschung at the University of Cologne, Aachener Str. 209, 50931 Cologne, Germany

5 ESTEC, ESA, Keplerlaan 1, 2200 AG, Noordwijk, The Netherlands 
our current knowledge. There have been no in-situ measurements of the deep Venus atmosphere since the flights of the two VeGa balloons and landers in 1985 (Sagdeev et al., Science 231:1411-1414, 1986). Thus, most of the new information about the atmospheric thermal structure has come from different remote sensing (Earth based and spacecraft) techniques using occultations (solar infrared, stellar ultraviolet and orbiter radio occultations), spectroscopy and microwave, short wave and thermal infrared emissions. The results are restricted to altitudes higher than about $40 \mathrm{~km}$, except for one investigation of the near surface static stability inferred by Meadows and Crisp (J. Geophys. Res. 101:4595-4622, 1996) from $1 \mu \mathrm{m}$ observations from Earth. Little information about the lower atmospheric structure is possible below about $40 \mathrm{~km}$ altitude from radio occultations due to large bending angles. The gaps in our knowledge include spectral albedo variations over time, vertical variation of the bulk composition of the atmosphere (mean molecular weight), the identity, properties and abundances of absorbers of incident solar radiation in the clouds. The causes of opacity variations in the nightside cloud cover and vertical gradients in the deep atmosphere bulk composition and its impact on static stability are also in need of critical studies. The knowledge gaps and questions about Venus and its atmosphere provide the incentive for obtaining the necessary measurements to understand the planet, which can provide some clues to learn about terrestrial exoplanets.

Keywords Venus $\cdot$ Atmosphere $\cdot$ Thermal structure $\cdot$ Radiative balance

\section{Introduction}

The thermal structure of the Venus atmosphere, extending more than two hundred km above the surface, is a result of the radiative and convective processes, which are governed by the scattering and absorption of the incident solar radiation and of the infrared emitted radiation from the surface and the atmosphere. In this paper, we focus below about $200 \mathrm{~km}$ due to paucity of observations above $130 \mathrm{~km}$. One might anticipate that with a very low obliquity spin axis, circular orbit, ubiquitous cloud cover and no oceans, the Venus atmosphere would be relatively easy to understand with regard to its thermal structure, radiative balance and circulation. The global atmospheric thermal structure is key to the global atmospheric circulation and the structure of the global cloud cover. The global thermal structure of the deep Venus atmosphere, extending nearly two hundred $\mathrm{km}$ above the surface, is a result of the radiative and convective processes. Thus understanding the thermal structure requires a knowledge of the absorption of the incident solar radiation and escape to space of the emitted infrared radiation from the surface and the atmosphere itself. Both the absorption and escape of radiation depend on the cloud structure and the chemical composition of the atmosphere, which have been reviewed in the companion papers by Titov et al. (2018) and Marcq et al. (2018) respectively.

Variations of temperature with latitude or longitude on a pressure or altitude level tend to be small on Venus compared to Earth, as we have learned from observations from more than a dozen atmospheric entry probes, two balloons, and many orbiters (Venera 9, 10, 15, and 16, Pioneer Venus, Magellan, Venus Express and Akatsuki (observing since December 2015). The results of these missions still have not been able to solve one of the continuing puzzles about Venus - the rapid rotation of its atmosphere, much faster than the underlying planet at all altitudes and latitudes where accurate measurements have been made (above $\sim 5 \mathrm{~km}$ altitude). This circulation, believed to be in cyclostrophic balance (Leovy 1973 ) is driven by meridional pressure differences (Limaye 1990; Newman et al. 1984). 
The measured latitudinal thermal structure shows that the pole-equator temperature differences at altitudes up to $65 \mathrm{~km}$ are relatively small, $\sim 10 \mathrm{~K}$ (Kliore 1985, Seiff et al. 1985). Longitudinal differences from surface to the cloud tops $(\sim 70 \mathrm{~km})$ are $<5 \mathrm{~K}$ in low latitudes (Seiff et al. 1980; Kliore 1985) and can be $\sim 20 \mathrm{~K}$ in higher latitudes $\left(>60^{\circ}\right)$. In the deep atmosphere, at $10 \mathrm{~km}$ altitude, winds are as fast as $5 \mathrm{~m} \mathrm{~s}^{-1}$ (Counselman et al. 1980), suggesting at least some meridional temperature gradient must exist if the flow is cyclostrophic. Given the large thermal inertia, the temperature differences in the near surface atmosphere are expected to be small in the deep atmosphere (Stone 1975). The two VeGa balloons sampled different portions of the atmosphere at $\sim 54 \mathrm{~km}$ two days apart while moving in nearly zonal trajectories at $5^{\circ} \mathrm{N}$ and $6^{\circ} \mathrm{S}$ latitudes but showed a consistent $6 \mathrm{~K}$ difference in temperatures (Sagdeev et al. 1986). Mueller et al. (2018) suggest regional variations in surface temperatures from analysis of near infrared observations from Venus Express.

For the 40-80 km layer, radio occultations provide profiles of thermal structure with higher vertical resolution $(\sim 500$ meters) but relatively sparse latitude-longitude coverage compared to the passive infrared retrievals from Fourier Spectrometer on Venera 15 orbiter and Visual and Infrared Thermal Imaging Spectrometer (VIRTIS) experiment on Venus Express orbiter. The infrared retrievals have lower vertical resolution $(\sim 2.5 \mathrm{~km})$ due to the width of the weighting functions of the spectral channels of the respective instruments but provide good spatial coverage. Nearly a decade of monitoring the planet from Venus Express and continuing observations from Akatsuki orbiter have expanded the vertical and temporal coverage of observations to the thermal structure results.

Above $80 \mathrm{~km}$ altitude, the thermal structure has been inferred from the drag effects of the entry probes (Avduevskii et al. 1983a, 1983b; Seiff and Kirk 1982), the Pioneer Bus and orbiter drag data and near infrared solar and ultraviolet stellar occultations from Venus Express orbiter and from ground based infrared observations as discussed by Limaye et al. (2017).

The results of thermal structure investigations available until early 1980s were reviewed by Seiff (1983) and used in the Venus International Reference Model (VIRA) described by Seiff et al. (1985). Radio occultations from Magellan (Jenkins et al. 1994) and Venera 15 and 16 orbiters (Yakovlev et al. 1987) and retrievals from the Fourier Spectrometer infrared observations from Venera 15 have been incorporated by Zasova et al. (2006) in an interim update of the VIRA model (Seiff et al. 1985). These and ground based results were summarized by Lellouch et al. (1997). Subsequently Venus Express provided extensive thermal structure results from several experiments during 2006-2014 (Drossart and Montmessin 2015). The Venus Express mission obtained data on the Venus atmosphere from 11 April 2006 until 27 November 2014, spanning about 13 Venus days, 14 Venus years or 27 Venus solar days. The Venus Express results extended the vertical coverage to about $200 \mathrm{~km}$. The structure between 100-200 km altitude was previously investigated only by ground-based investigations (which have relative low horizontal resolution and coverage) and from Pioneer Venus Entry Probe Bus and deceleration of the Pioneer entry probes (Seiff and Kirk 1982). The Venus Express results and new ground-based observations were inter compared towards updating the Venus International Reference Atmosphere (Kliore et al. 1985) by Limaye et al. (2017).

Akatsuki orbiter succeeded on its second attempt to enter into orbit around Venus on 7 December 2015 and has been observing the planet routinely since beginning of April 2016 (Nakamura et al. 2007, 2014, 2016). From its low inclination orbit, Akatsuki provides more low latitude radio occultation profiles (but fewer due to its 10.5-day orbit) compared to the high latitude coverage from Venus Express from its 24 hour polar orbit. Akatsuki results 
on the Venus cloud cover have been presented by Limaye et al. (2018a), the thermal infrared results by Fukuhara et al. (2017) and initial thermal structure $(35-90 \mathrm{~km})$ results from radio occultations are described by Imamura et al. (2017).

Additionally, experimental and theoretical investigations of mixtures of super critical gases are encouraging some earlier puzzling observational results such as the unexplained vertical gradient of nitrogen abundance in the atmosphere (Oyama et al. 1980) and the presence of a puzzling unstable layer below $7 \mathrm{~km}$ altitude seen in the VeGa 2 lander data (Lebonnois and Schubert 2017) to be revisited. The LIR camera on Akatsuki orbiter (Taguchi et al. 2017) is providing continuous cloud top brightness temperatures and raising more questions. There are remaining questions about both the thermal structure and the radiative balance. The VeGa 2 Lander remains the only probe that has returned reliable measurements of the thermal structure below $12 \mathrm{~km}$ altitude (Linkin et al. 1986a, 1986b) on June 15, 1985 at $8.5^{\circ} \mathrm{S}, 145.8^{\circ} \mathrm{E}$ and $164.5^{\circ}$ solar zenith angle.

Energy balance within a planetary atmosphere is determined by the distribution of temperature as well as opacity sources. Wildt (1940) considered the impact of a large amount of $\mathrm{CO}_{2}$ on the surface temperature of Venus long before spacecraft data was available. Sagan (1960) followed up the Venus atmosphere energy with an in-depth study of the water vapor abundance in the atmosphere and the greenhouse effect and considering high value of the radio measurements of temperature (Mayer et al. 1958) as possibly being thermal emission. On Venus, about half of the absorption of incident sunlight takes place in the clouds. While it is known that sulfur dioxide absorbs below about $330 \mathrm{~nm}$, the identity of the other absorbers, which must be present in the clouds, is an unsolved mystery and recently Limaye et al. (2018b) explored whether some of the absorption can be by colonies of microorganisms in the habitable zone in the cloud layer. Radiative balance of the Venus atmosphere has been discussed previously by Tomasko et al. (1980b), Crisp and Titov (1997) and by Titov et al. (2007, 2013). Tomasko et al. (1980b) reviewed the Pioneer and Venera probes results while Crisp and Titov (1997) addressed the need for unidentified opacity sources to explain the high surface temperatures, the effects of lower water vapor amounts and cloud opacities on the atmosphere and the anomalously warm mesosphere. The radiative balance in the context of the climate of Venus was discussed by Titov et al. (2013) while Titov et al. (2007) discussed the energy and entropy budget of the Venus atmosphere. They pointed out that the Carnot cycle efficiency of the Venus heat engine is about twice as large (27.5\%) as that for Earth (Titov et al. 2007; Schubert and Mitchell 2013), suggesting a different distribution for the transfer of energy within the planet's atmosphere as compared to Earth. The Carnot efficiency for Earth has been estimated from an extensive thermodynamic analysis using a climate model (Lucarini et al. 2010) and estimated to be considerably less than quoted by Schubert and Mitchell. It is not known whether the discrepancy is due to the approach or due to the use of the model rather than the simplistic definition used previously.

Haus (2017) has published a parameterization scheme to calculate the cooling and heating rates in the atmosphere including the impact of the unknown absorbers of sunlight in the Venus clouds on the energy balance. Schubert and Mitchell (2013) reviewed the energy balance of Venus in a comparative aspect with other planets and concluded that the entropy production rate in the Venus atmosphere is only slightly less $\left(23 \mathrm{~mW} \mathrm{~m}^{-2} \mathrm{~K}^{-1}\right)$. This is comparable to that for Earth $\left(29 \mathrm{~mW} \mathrm{~m}^{-2} \mathrm{~K}^{-1}\right)$ and much higher compared to Mars $\left(1 \mathrm{~mW} \mathrm{~m}^{-2} \mathrm{~K}^{-1}\right)$ and Titan $\left(0.1 \mathrm{~mW} \mathrm{~m}^{-2} \mathrm{~K}^{-1}\right)$ as estimated from the excess latent and sensible heat exchange between surface and the atmosphere. Read et al. (2016) present a similar and independent assessment. Below we present the status of our knowledge of the Venus atmospheric thermal structure and radiative balance that incorporates recent observations. 
We note that recent results on the atmospheric circulation are discussed in depth recently by Sánchez-Lavega et al. (2017), the atmospheric chemistry by Marcq et al. (2018), while the cloud structure results are summarized by Titov et al. (2018). In this paper, we present an overview of the thermal structure observations (Sect. 2) then present a summary of recent investigations of the thermal structure (Sect. 3). The vertical thermal structure is presented in Sects. 4 and 5 describes the radiative balance of Venus. Section 6 discusses open issues, knowledge gaps and future investigations about the thermal structure and radiative balance.

\section{Investigations of the Thermal Structure of the Venus Atmosphere}

First clues about the thermal properties came from radio measurements of temperature (Mayer et al. 1958) at $3.15 \mathrm{~cm}$, which indicated $600 \mathrm{~K}$ temperature. Radio measurements at other wavelengths (Kuzmin 1983) also were higher compared to the spectroscopic or bolometric temperatures (Pettit and Nicholson 1955; Strong and Sinton 1960) leading to a debate whether the high temperatures were ionospheric or surface. The debate was resolved by the microwave radiometer observations from Mariner 2 in favor of the high surface temperature, confirmed later by Venera and Pioneer Venus entry probes (only up to $12 \mathrm{~km}$ due to electrical failure). In 1985, VeGa 2 became the first probe to measure the temperature below $12 \mathrm{~km}$ down to the surface (Linkin et al. 1986a, 1986b).

The radio occultation technique $\mathrm{X}$ and $\mathrm{S}$ band frequencies was used to obtain temperature profiles of the upper atmosphere (35 to $90 \mathrm{~km}$ ) of Venus for the first time from Mariner 5 (Kliore et al. 1967). These were followed by Mariner 10 (Fjeldbo et al. 1971), Pioneer Venus orbiter (Kliore and Patel 1980), Magellan (Jenkins et al. 1994; Jenkins 1998), Venus Express (Tellmann et al. 2009) and now Akatsuki (Imamura et al. 2017). Since Magellan orbiter's main mission was radar mapping of the Venus surface, the radio occultation sessions were not routinely conducted as it used the antenna for both the radar experiment and communications with the Earth receiving stations. The few occultations (21) obtained are some of the best obtained. Thermal infrared measurements of the Venus upper atmosphere from Earth based telescopes have also been obtained in recent years (Clancy et al. 2012; Sato et al. 2014; Piccialli et al. 2017). Venus has been observed at meter wavelengths also recently (Mohan et al. 2017) extending the radio frequency coverage.

Venus Express became the first orbiter around the planet armed with multiple instruments to measure the thermal structure of the atmosphere - Visible InfraRed Thermal Imaging Spectrometer (VIRTIS), Spectroscopy for the Investigation of the Characteristics of the Atmosphere of Venus (SPICAV) and Solar Occultation in the InfraRed (SOIR), and a Venus Radio Science Investigation (VeRa). These experiments have provided a vertical and spatial coverage of the temperature structure of the atmosphere over the duration of the mission. In addition, the Venus Express Atmospheric Drag Experiment (VEXADE) conducted by Döppler tracking of the spacecraft during it occasional dips into the atmosphere during periapsis passages. The resulting atmospheric drag, has provided useful data on the altitude (and solar zenith angle) dependence of atmospheric density at altitudes between 130-200 km. Pioneer Venus orbiter also provided atmospheric structure data from drag during periapsis passages (Niemann et al. 1980) and at the end of its life (Kasprzak et al. 1993). The observations provide considerable horizontal but limited vertical overlap. VeRa at microwave wavelengths (X- and S-band) and all local solar times, as well as SOIR at the terminator and SPICAV on the night side at infrared and ultraviolet wavelengths, respectively, used the occultation 


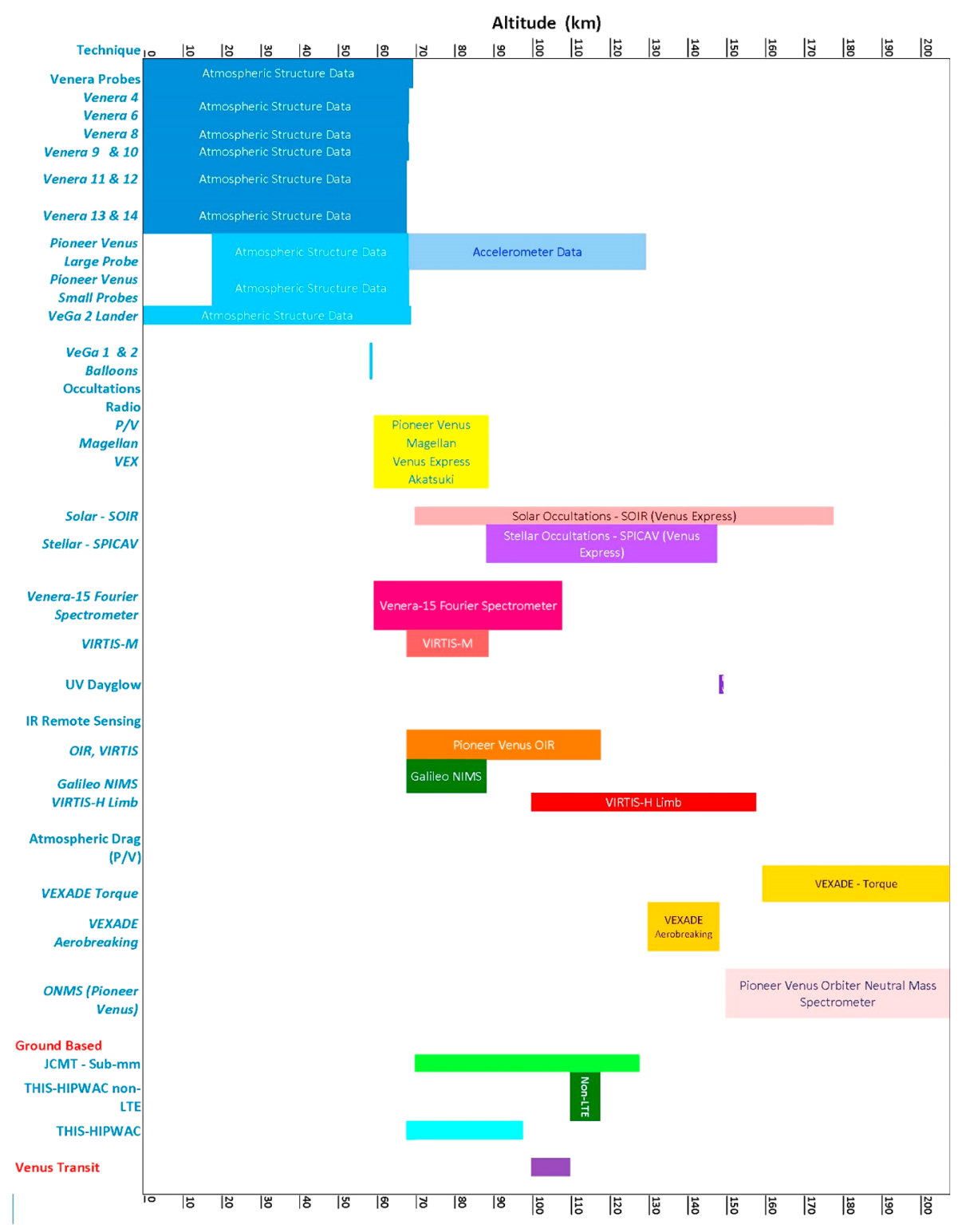

Fig. 1 Vertical coverage of thermal structure results from different experiments to date that have provided significant information about the thermal structure of the Venus atmosphere. Techniques or spacecraft are given on the vertical axis and the altitude coverage is on the horizontal axis

technique at radio, short infrared and ultraviolet wavelengths respectively, to sample the atmosphere ranging from $40 \mathrm{~km}$ (VeRa) and $170 \mathrm{~km}$ (SOIR). SPICAV results at the vertical extremes overlap with VeRa and SOIR measurements. Figure 1 shows the altitude regions sampled by all the different experimental approaches, during the lengthy Venus exploration. Each experimental approach has different spatial and temporal sampling, intrinsic limits, errors and coverage. 
Table 1 Summary of observations of the atmospheric thermal structure from Venus Express

\begin{tabular}{|c|c|c|}
\hline Experiment & $\begin{array}{l}\text { Altitude } \\
\text { range }\end{array}$ & Characteristics \\
\hline $\begin{array}{l}\text { VeRa radio } \\
\text { occultation }\end{array}$ & $40-85 \mathrm{~km}$ & $\begin{array}{l}\text { X-and S-band radio occultation and high vertical resolution }(\sim 500 \mathrm{~m}) \text {, } \\
\text { derived via index of refraction and bending angle }\end{array}$ \\
\hline VIRTIS-M & $62-82 \mathrm{~km}$ & $\begin{array}{l}\text { Inversion of spectral measurements, low vertical resolution }(\sim 1 \text { scale } \\
\text { height) but with large horizontal coverage due to mapping mode on } \\
\text { night side }(1-5 \mu \mathrm{m})\end{array}$ \\
\hline VIRTIS-H & $64-80 \mathrm{~km}$ & $\begin{array}{l}\text { Inversion of spectral measurements, low vertical resolution }(\sim 1 \text { scale } \\
\text { height), only at nadir locations on night side }(2-5 \mu \mathrm{m}) \text {. Better coverage } \\
\text { of equatorial and northern latitudes with respect to VIRTIS-M }\end{array}$ \\
\hline \multicolumn{3}{|l|}{$\begin{array}{l}\text { (Thermal } \\
\text { emission) }\end{array}$} \\
\hline $\begin{array}{l}\text { VIRTIS-H } \\
\text { (Non-LTE) }\end{array}$ & $100-150 \mathrm{~km}$ & Day time Non-LTE analysis of limb and nadir observations $(1-5 \mu \mathrm{m})$ \\
\hline SOIR & $70-170 \mathrm{~km}$ & $\begin{array}{l}\text { Solar occultation (IR), high vertical resolution, derived by extinction of } \\
\text { solar signal measured over the occultation, provides good absorber } \\
\text { density }(2.3-4.2 \mu \mathrm{m})\end{array}$ \\
\hline SPICAV & $75-120 \mathrm{~km}$ & $\begin{array}{l}\text { Stellar occultation, derived by extinction of stellar signal measured over } \\
\text { the occultation }(0.11-0.31 \mu \mathrm{m})\end{array}$ \\
\hline $\begin{array}{l}\text { Atmospheric } \\
\text { drag }\end{array}$ & $165-300 \mathrm{~km}$ & $\begin{array}{l}\text { Direct estimate of ambient atmospheric density from measured torque } \\
\text { or force }\end{array}$ \\
\hline
\end{tabular}

One of the key contributions of Venus Express has been to provide information about the conditions above $100 \mathrm{~km}$ where only a limited number of measurements were previously available. These measurements and results are provided by SOIR, SPICAV, VIRTIS NonLocal Thermodynamic Equilibrium (LTE) emissions, and the atmospheric drag experiments using the spacecraft. Table 1 provides a summary of the major experiments from Venus Express, which have provided results on the Venus thermal structure. The earlier reviews through 1997 have generally focused on the results from the Pioneer Venus orbiter/probes, Venera probes, VeGa balloons/lander and other results, but have not addressed in detail experimental or analysis differences. We present below a summary of such differences in the next section. Venus Express observations continue to be analyzed and the results are being refined and compared. Numerical modeling efforts that are useful for understanding the observed thermal structure have been described by Lewis et al. (2013) and current numerical models of the Venus atmosphere are compared by Lebonnois et al. (2013).

\section{Recent Spacecraft and Earth Based Results of the Thermal Structure}

\subsection{Thermal and Non-LTE Emission Measurements}

\subsubsection{Pre-Venus Express Observations at Infrared Wavelengths}

Thermal structure measurements of the Venus atmosphere are now available from the surface to about $200 \mathrm{~km}$ altitude from a large number of different experiments and at different epochs and local solar time. Although the results of these measurements are generally consistent, providing us a good overview of the global and vertical structure, it is becoming 
apparent that the differences between them may be important, especially on the short term small and regional scale.

Taylor et al. (1980) presented the first results on the hemispheric structure of temperature profiles obtained from the Pioneer Venus Orbiter Infrared Radiometer - a filter radiometer comprised of eight infrared filters. Coverage was limited to the northern hemisphere due to 0.83 orbital eccentricity and $\sim 200 \mathrm{~km}$ periapsis altitude of the orbit with periapsis at low latitudes. The Fourier Spectrometer on the Venera 15 orbiter provided the first interferometric infrared observations of Venus at a moderately high spectral resolution between $250-1600 \mathrm{~cm}^{-1}(6-45 \mu \mathrm{m})$ with $5-7.5 \mathrm{~cm}^{-1}$ spectral resolution (Zasova et al. 1999) and the retrieved profiles were incorporated in an interim update to the Venus atmospheric thermal structure (Zasova et al. 2006). Pollack et al. (1993) presented a detailed study of the near infrared emission from the night side of Venus in the spectral windows discovered by Allen and Crawford (1984) which provided a basis for further investigations. Snels et al. (2014) have recently measured the $\mathrm{CO}_{2}$ opacity at high densities found in the lower atmosphere of Venus in the $1.18 \mu \mathrm{m}$ spectral window. Mondelain et al. (2017) report measurements in the $2.3 \mu \mathrm{m}$ window. These new measurements should help improve future analysis of observations in this window. The Galileo Orbiter yielded thermal structure results from the near infrared region of the spectrum during its gravity assist fly-by of Venus in February 1990 (Roos-Serote et al. 1995).

More than a decade later, the VIRTIS experiment on the Venus Express orbiter observed the planet in the near infrared region of the spectrum for more than two years and provided vertical temperature profiles on the night side from medium and high resolution spectral data in mapping and nadir modes as well as from day side limb observations.

\subsubsection{VIRTIS Experiment on Venus Express}

The Visual and Infrared Thermal Imaging Spectrometer (VIRTIS) on board Venus Express (see Piccioni et al. 2007a) provided observations of Venus from the near infrared at moderate spectral resolution. Notably, the VIRTIS spectral range covered the strong $4.3 \mu \mathrm{m}$ carbon dioxide band on both day and night sides. The night side data detected only the thermal emission from the atmosphere, whereas the day side measurements contained contributions from the scattered sunlight and solar-induced fluorescence. Different groups have analyzed the day and night side observations separately - Grassi et al. (2008, 2010, 2014); Migliorini et al. (2012); Haus et al. (2013, 2014) and by Garate-Lopez et al. (2015) using only the night-time data acquired at moderate emission angles $\left(>30^{\circ}\right.$, solar zenith angles $<95^{\circ}$ ). The variability of total opacity inside the $\mathrm{CO}_{2}$ band allowed retrieval of air temperatures from the cloud top level (about $100 \mathrm{mb}$, or $\sim 65 \mathrm{~km}$ at intermediate latitudes) up to $1 \mathrm{mb}$ $(\sim 85 \mathrm{~km})$, with an effective resolution in the order of 5-7 km (Grassi et al. 2008).

Gilli et al. (2015) and Peralta et al. (2016) have analyzed the day side measurements of the non-LTE emissions obtained from VIRTIS. The non-LTE emissions arise from the fluorescence of $\mathrm{CO}$ and $\mathrm{CO}_{2}$ in the thermosphere and provide valuable information in the $100-160 \mathrm{~km}$ altitude range where there is sparse coverage. Peralta et al. (2016) considered dayside nadir observations. In the most opaque parts of the $4.3 \mu \mathrm{m}$ band, the non-LTE emission induced by solar-fluorescence becomes the main source of signal, allowing one to constrain the kinetic temperatures in the lower thermosphere $(\sim 120 \mathrm{~km})$. Non-LTE emissions have been exploited in the analysis of limb dayside measurements presented by Gilli et al. (2015), who used the $4.7 \mu \mathrm{m}$ carbon monoxide band to obtain vertical profiles of CO and temperature. 


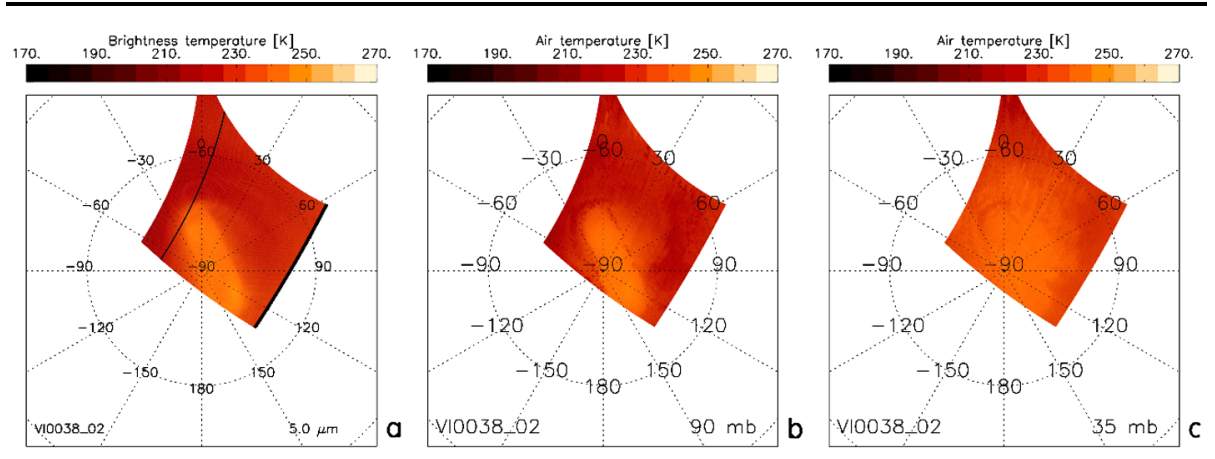

Fig. 2 Analysis of VIRTIS-M cube VI0038_02. Panels a-c present the radiance measured at $5 \mu \mathrm{m}$ and the temperatures retrieved at the levels of $90 \mathrm{mb}$ and $35 \mathrm{mb}$ respectively. (From Grassi et al. 2008.) The vertical dependence of the night time temperatures is shown in Fig. 6

VIRTIS Results from Thermal Emissions Results from the measured thermal emission (night side) are presented in Fig. 2. The results shown have been obtained from measurements subject to a few observational constraints (exposure time, emission angles), and were acquired by the imager channel of the instrument (VIRTIS-M), operating at moderate spectral resolution (spectral resolving power of 100-200). The elliptic (eccentricity $=0.8$ ) orbit of Venus Express resulted in good coverage of the southern polar region. Coverage of the northern hemisphere was more sparse since the VIRTIS field of view observed the Venus globe in narrow stripes along different latitudes acquired when Venus Express passed closer to the periapsis.

Grassi et al. (2008) presented a first exploratory study of Venus temperature fields from VIRTIS-M night side data in the 4.3-4.8 $\mu \mathrm{m}$ range. Numerical experiments on simulated observations allow one to set an estimate for retrieval errors to be $2-3 \mathrm{~K}$. The near cloud top temperature retrievals depend on the assumed aerosol distribution and aerosol properties. Despite this uncertainty, and the "cold collar" and the "dipole structure" previously seen between the pole and $\sim 75^{\circ} \mathrm{N}$ in the Pioneer Venus data (Taylor et al. 1980) have been seen in the VIRTIS-derived maps at the cloud top level, about $90 \mathrm{mb}$ in the southern hemisphere. The dipole has been shown to be just one shape out of many manifestations of the arising out of dynamical instability within the core of the hemispheric vortex (Garate-Lopez et al. 2015; Limaye et al. 2009). Maximum inferred temperatures are around $240 \mathrm{~K}$. At the same pressure levels, the cold collar at $75^{\circ} \mathrm{S}$ is also observed, with a temperature drop of at least $10 \mathrm{~K}$ with respect to the dipole. The cold collar pattern rapidly disappears at higher levels. Already at $35 \mathrm{mb}$, temperatures tend to decrease monotonically from the pole toward the equator. Comparison of different maps acquired in close temporal sequence (about $60 \mathrm{~min}$ ) suggests that short time variability is maximum (about $5 \mathrm{~K}$ ) around the $1 \mathrm{mb}$ level, in the region $65^{\circ} \mathrm{S}-75^{\circ} \mathrm{S}$ and on the dawn side of the night hemisphere.

Grassi et al. (2010) extended this analysis, considering a wider set of data and presenting average temperature fields in the local time/latitude/pressure space (Fig. 3) and their variability. The most evident feature is the strong dusk/dawn asymmetry observed in average fields at the $100 \mathrm{mb}$ level. The cold collar regions present a minimum temperature of about $215 \mathrm{~K}$ at $3 \mathrm{AM} \mathrm{LT}$ and $65^{\circ} \mathrm{S}$. Moving to higher altitudes at the same latitude, temperature initially tends to decrease from dusk to dawn $(35 \mathrm{mb})$, but becomes more uniform in local time around $12 \mathrm{mb}$ and eventually becomes warmer on the dawn side at $4 \mathrm{mb}$. With such large averages, standard deviation of temperatures is no longer representative of short term variations in the atmosphere. With respect to the discussion presented in Grassi et al. 

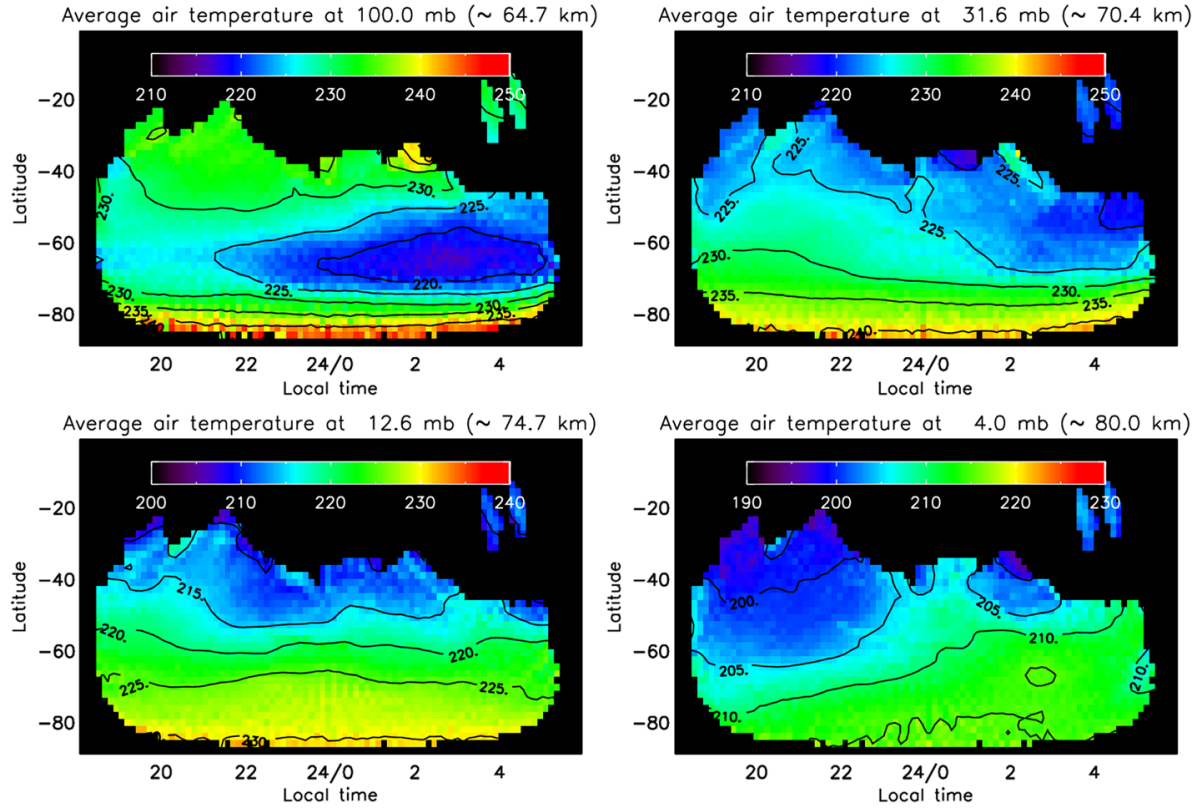

Fig. 3 Average temperature fields in the Venus nighttime southern hemisphere, as derived from the analysis of a large sample of the VIRTIS-M dataset. Panels a-d refer to the levels of 100, 31.6, 12.6 and $4.0 \mathrm{mb}$. From Grassi et al. (2010). See also Fig. 6 that shows the vertical dependence of the nighttime temperatures from Haus et al. (2014)

(2008), other phenomena become evident. The largest variabilities $(10 \mathrm{~K})$ are observed at the $100 \mathrm{mb}$ level around $80^{\circ} \mathrm{S}$, at the boundary between the polar dipole and cold collar. This variability likely reflects the variability in the position of the dipole lobes and of the small scale structures observed in this region. More interestingly, the region of minimum temperature in the cold collar at $100 \mathrm{mb}$ and 3:00 LT is also characterized by a high variability $(6-8 \mathrm{~K})$, previously unreported. At higher levels, maximum variability again appears at the $1 \mathrm{mb}$ level around 1:00 LT and possibly higher on the dawn side. This trend is quantitatively consistent with earlier suggestions in Grassi et al. (2008). The vertical dependence of the night time temperatures was also examined by Haus et al. (2014) and is discussed later (Fig. 7).

Migliorini et al. (2012) presented similar average fields derived from a more numerically limited dataset in the high spectral resolution channel of VIRTIS (VIRTIS-H). This channel returned a smaller number of spectra but offers a better coverage of the equatorial and northern latitudes (Fig. 4). From these measurements, a cooler cold collar toward dusk is also possibly seen in the northern hemisphere, but the improved coverage is insufficient for a firm conclusion. In equatorial regions up to latitudes of $50^{\circ}$ in both hemispheres, temperatures are higher on the dusk side with respect to the dawn side at $100 \mathrm{mb}$ and show a broad local minimum at 4:00 LT at all pressure levels between 80 and 10. This confirms previous observations of Pioneer Venus OIR (Taylor et al. 1980). Moreover, it should be noted that air temperatures display very modest variations along latitudes at fixed local times in the large region $50^{\circ} \mathrm{S}-50^{\circ} \mathrm{N}$. For pressures lower than $10 \mathrm{mb}$, estimates from VIRTIS-H appear systematically higher than the ones derived from VIRTIS-M and OIR. Recent re-analysis of VIRTIS-H data suggests that this is probably due to a calibration residual in the weakest part of the $\mathrm{CO}_{2}$ band (Grassi 2016, personal communication). 
Fig. 4 Average temperature fields in the Venus nighttime hemisphere at the 100 (panel a) and 31 (panel b) mb levels, as derived from the analysis of a large sample of the VIRTIS-H dataset. (From Migliorini et al. 2012)
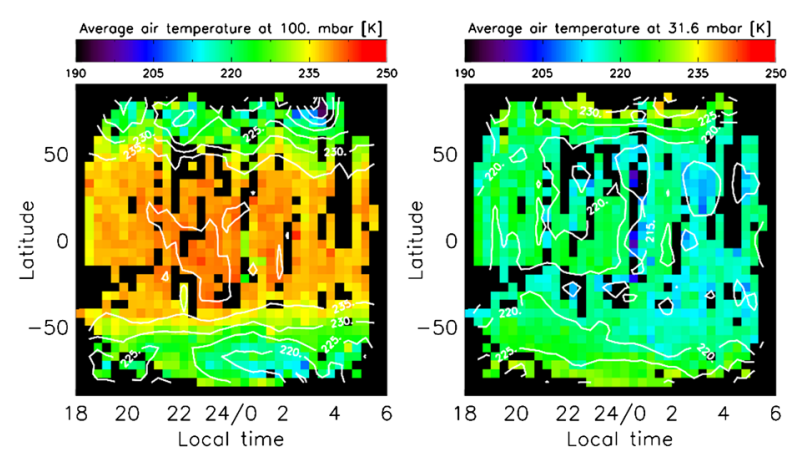

Fig. 5 Average temperature latitude-altitude cross section in the Venus nighttime hemisphere, as derived from analysis of the VIRTIS-M dataset. (From Haus et al. 2014)

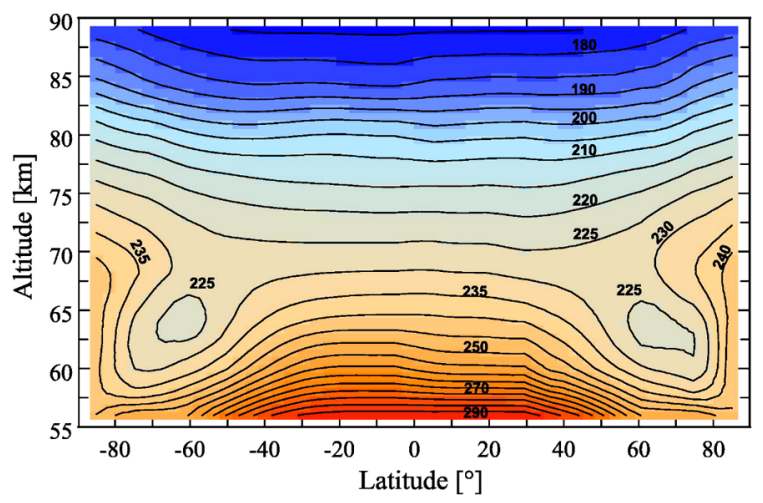

Grassi et al. (2014) expanded the analysis of average fields by considering all suitable measurements acquired by VIRTIS-M during its operative lifetime. With respect to Grassi et al. (2010), the re-analysis also benefited from a new retrieval scheme that allows one to consistently incorporate the treatment of variable cloud deck altitude as well as the retrieval of carbon monoxide content. Results of Grassi et al. (2010) are essentially confirmed: more stringent acceptance criteria resulted in a wider cold collar region (polar dipole demonstrated it is particularly difficult to model with the new retrieval algorithm), while improved confidence allowed the authors to discuss an average field at the 1.4 bar level, that turned out to be roughly symmetric around midnight.

Haus et al. (2013) also analyzed VIRTIS-M data independently and presented an improved treatment of the VIRTIS-M data, using a more complete treatment of aerosols, made possible by including the 2.3 and $1.74 \mu \mathrm{m}$ radiation measured by VIRTIS-M in the retrieval process. Moreover, the authors developed a sophisticated pre-processing pipeline that allowed Haus et al. to improve radiometric accuracy of the measurements prior to retrieval. Removal of residual stray light contributions on the spectra and further corrections of nonuniform response along the slit provided particularly beneficial results (Kappel et al. 2012). These methods allowed Haus et al. to reduce the retrieval uncertainties at the cloud top level and to provide reliable retrievals down to the $60-55 \mathrm{~km}$ level. Including the few measurements covering the northern hemisphere are effectively exploited to create an average latitudinal-altitude cross section shown in Fig. 5. The resulting field is remarkably symmetric around the equator, essentially reproducing the structure obtained from the Pioneer Venus and Venera 15 results which also agree with the Grassi et al. (2014) results and also similar to the structure seen from radio occultation results from Pioneer Venus (Seiff et al. 
Fig. 6 Average temperature local time-altitude cross sections at $65^{\circ} \mathrm{S}$ (panel a) and $75^{\circ} \mathrm{S}$ (panel b), as derived from the analysis of the VIRTIS-M dataset. (From Haus et al. 2014)

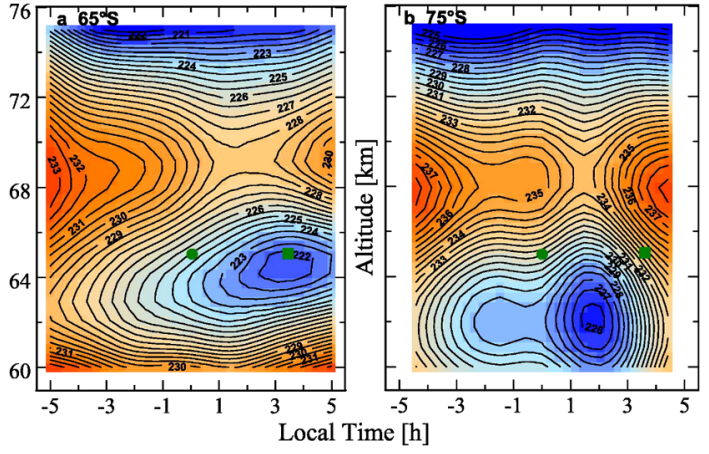

Fig. 7 Air temperature standard deviations in the Venus nighttime southern hemisphere, as derived from the analysis of the VIRTIS-M dataset. (From Haus et al. 2014)

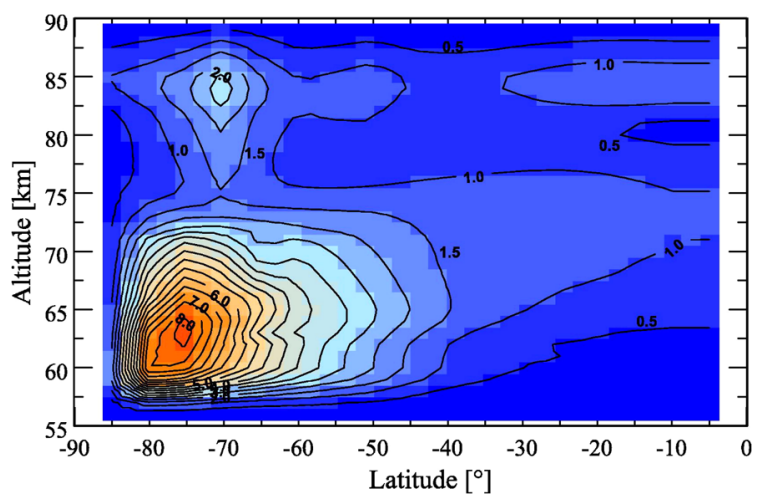

1985) and Venus Express (Sect. 3.2.3). It can be seen that the cold collar does not extend deep into the atmosphere and that already around $55 \mathrm{~km}$ temperatures increase, moving from the pole to the equator, and confirming previous similar finding from the FTS-Venera 15 data (Zasova et al. 1999). In the high altitude regions above $65 \mathrm{~km}$, where comparison against Grassi et al. (2008) and Grassi et al. (2010) is possible, the agreement between the two teams' results remains within a few degrees. Grassi et al. (2010) results above $4 \mathrm{mb}$ are slightly warmer and are believed to be due to residual stray light in the original calibrations, similar to the one mentioned above for VIRTIS-H.

The extensive coverage from VIRTIS also enables examination of the local solar time structure with altitude. Two examples are shown in Fig. 6 (for $65^{\circ} \mathrm{S}$ and $75^{\circ} \mathrm{S}$ latitudes). Asymmetry of the temperature minimum in the cold collar is confirmed by this analysis: the region centered at $65^{\circ} \mathrm{S}, 3: 00 \mathrm{AM} \mathrm{LT}, 65 \mathrm{~km}$ presents a minimum temperature below $222 \mathrm{~K}$, while closer to the pole, the center of the cold region moves closer to midnight (1:00 AM LT) and deeper in the atmosphere by a few kilometers. Analysis of air temperature variability (Fig. 7) confirms that the cold collar is characterized by strong variations up to $10 \mathrm{~K}$.

Garate-Lopez et al. (2015) independently developed another retrieval scheme to estimate air temperatures from the $4.3 \mu \mathrm{m} \mathrm{CO}_{2}$ band in VIRTIS-M nighttime data. Their analysis is noteworthy for the extensive discussion on specific structures of the polar vortex and cold collar and the variability between different observations (Fig. 8). Retrieved temperature profiles are discussed down to $55 \mathrm{~km}$, also taking into consideration the lower cloud top altitude in the polar regions. The cold collar region is seen to display strong variations in its shape, and very fine structures in the dipole and at the boundary of the cold collar are demonstrated to be of common occurrence. Static stability $(S=\Gamma-d T / d z, \Gamma$ is the adiabatic lapse rate 

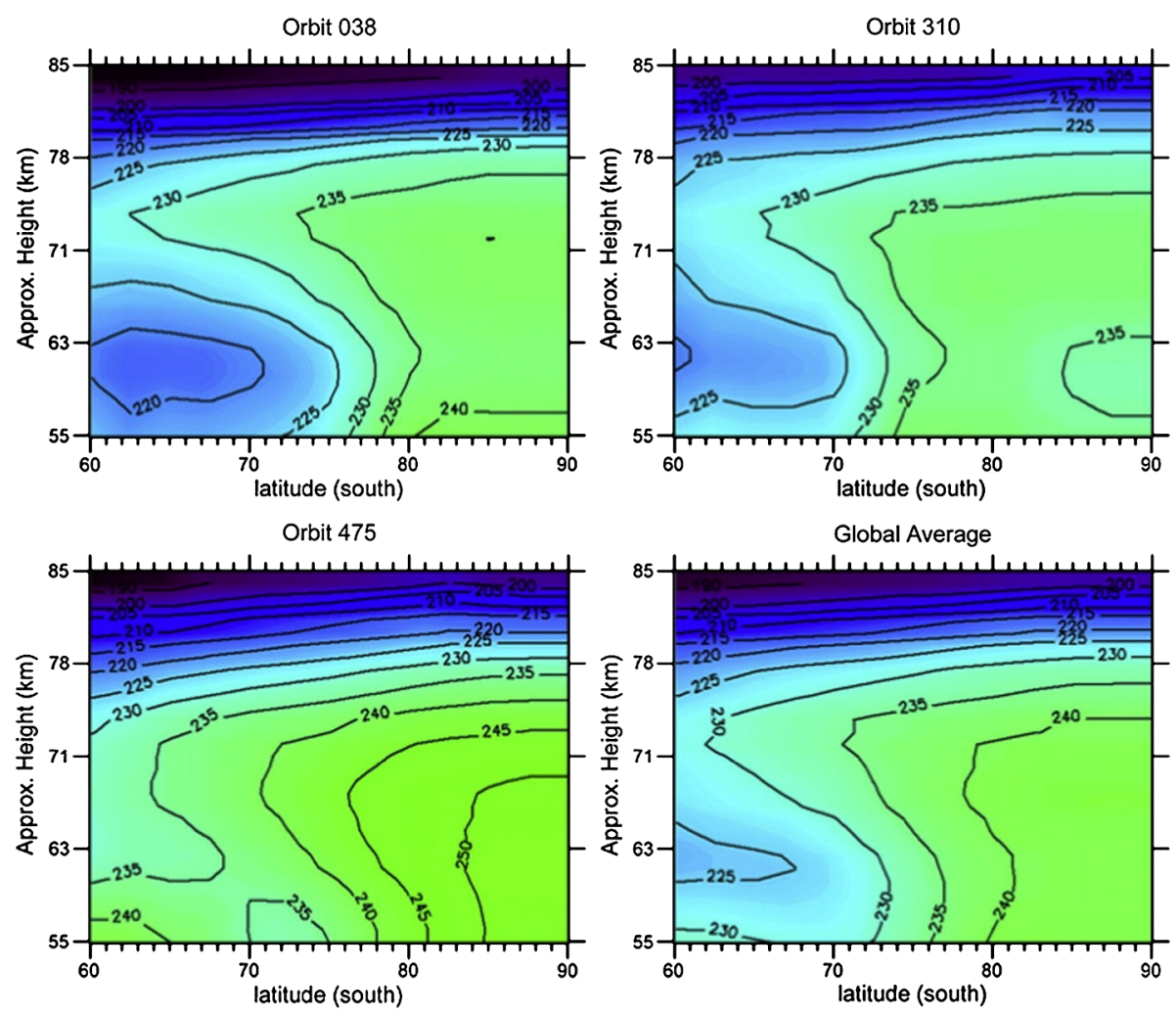

Fig. 8 Air temperature latitude -altitude cross section in the region of polar dipole and cold collar, from several individual VIRTIS-M cubes. Global average is also shown for comparison. (From Garate-Lopez et al. 2015)

and $d T / d z$ the rate of change of temperature, $T$ with altitude, $z$ ) is always found to be positive in the entire pressure range sampled by retrievals. More stable $\left(S>14 \mathrm{~K} \mathrm{~km}^{-1}\right)$ regions coincide with colder parts of the cold collar around $65 \mathrm{~km}$, while bright filaments at the boundary of the dipole lobes show lower stability, but are always clearly positive $\left(8-10 \mathrm{~K} \mathrm{~km}^{-1}\right)$.

Day Side VIRTIS Temperature Profiles from Non-LTE Emissions Vertical profiles of $\mathrm{CO}$ abundance and temperature have been obtained by Gilli et al. (2015) from an analysis of $\mathrm{CO}$ non-LTE emission at $4.7 \mu \mathrm{m}$ from the daytime limb spectra acquired by VIRTIS-H. The retrieval process is iterative and requires use of lower boundary values and the use of hydrostatic equilibrium. The results are subject to retrieval errors between 30 and $60 \mathrm{~K}$ (increasing with altitude), but are adequate to define some general trends (Fig. 9). Temperature generally tends to increase with altitude, rising from $150-180 \mathrm{~K}$ at $105 \mathrm{~km}$ to $220-250 \mathrm{~K}$ at $140 \mathrm{~km}$. Gilli et al. (2015) results suggest a local maximum around $115 \mathrm{~km}$ near the terminator at equatorial latitudes, but not at noon.

Peralta et al. (2016) present an analysis of non-LTE emission from the center of the $\mathrm{CO}_{2} 4.3 \mu \mathrm{m}$ band observed in nadir from daytime VIRTIS-H measurements. In the most opaque portions of the band, thermal contribution from the lower atmosphere is negligible and the observed signal is driven solely by the solar fluorescence originated in the thermo- 

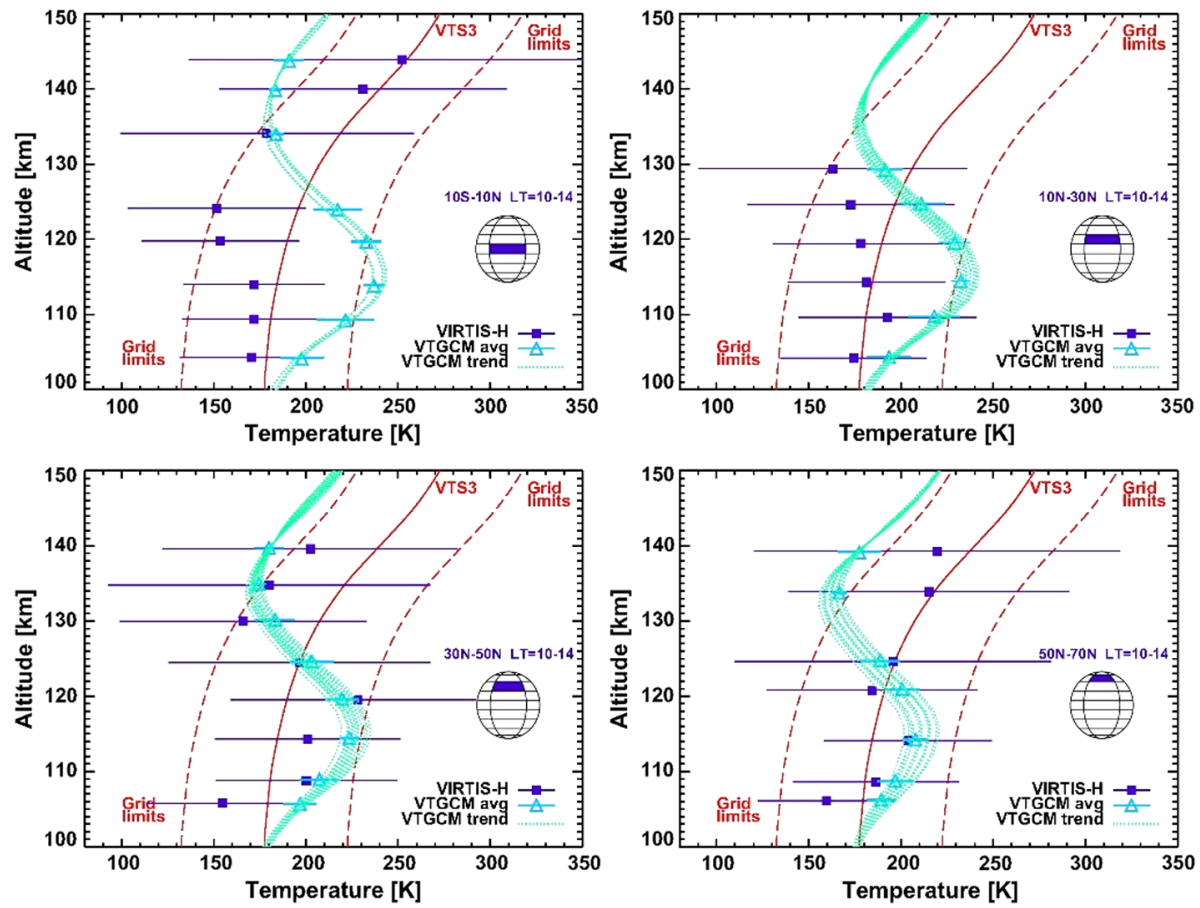

Fig. 9 Retrievals of kinetic temperature and error bars, at local time 10-14 h, as a function of altitude, from VIRTIS-H limb averages. Results at four latitudes are shown in the four panels, as indicated in the legends: $10^{\circ} \mathrm{S}-10^{\circ} \mathrm{N}$ (top-left panel), $10^{\circ} \mathrm{N}-30^{\circ} \mathrm{N}$ (top-right), $30^{\circ} \mathrm{N}-50^{\circ} \mathrm{N}$ (bottom-left) and $50^{\circ} \mathrm{N}-70^{\circ} \mathrm{N}$ (bottom-right). VTGCM (Bougher et al. 2015) daytime average temperature, corresponding to the VIRTIS-H selected boxes, are shown with their error bars (purple squares and lines). A set of profiles from the VTGCM are also shown with dotted-green lines, labeled as "VTGCM trend", which are individual outputs of the VTGCM within the VIRTIS latitude-local time bins. The VTS3 profile (solid red line), and the grid limits (dashed red lines) are shown for reference. (From Gilli et al. 2015)

sphere. While the method is sensitive to the air temperature in the $10^{-2}-10^{-5} \mathrm{mb}$ range $(\sim 105-145 \mathrm{~km})$, sensitivity is strongly peaked around $5 \times 10^{-4} \mathrm{mb}(\sim 120 \mathrm{~km})$. Here, retrievals are subject to an error of 5-10 K. Peralta et al. (2016) present average local timelatitude maps for the daytime hemisphere (Fig. 10). Maximum temperatures of $190 \mathrm{~K}$ are found to occur around the subsolar point, and decrease toward the terminator to $140 \mathrm{~K}$. These estimates are found to be in excellent agreement with the independent assessment of Gilli et al. (2015). Variability inside each averaging bin is usually on the order of the retrieval error, but increase up to $20-25 \mathrm{~K}$ towards the terminator, therefore indicating more active regions. Temperatures do not show obvious long time trends, but there are a few instances of sudden rises up to $30 \mathrm{~K}$, that cannot immediately be correlated with the solar radio flux.

Several investigations of the VIRTIS data are still ongoing. Most notable among them are the study of daytime nadir data (that requires simultaneous modeling of solar scattering and fluorescence) and possible assessment of temperature variability below the cloud deck from analysis of thermal emission observations. 
Fig. 10 Average temperature field in the Venus thermosphere around $5 \times 10^{-4} \mathrm{mb}$, as inferred from VIRTIS-H nadir observations of $\mathrm{CO}_{2}$ non-LTE emission on the dayside. From Peralta et al. (2016)

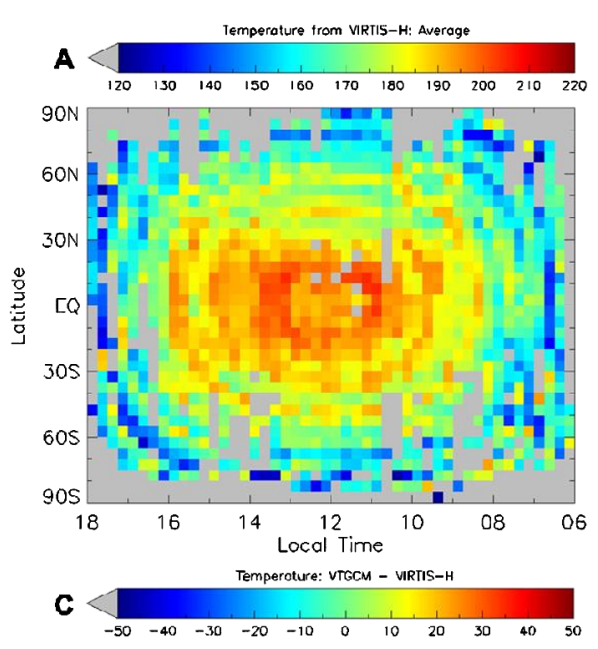

\subsection{Occultation Methods}

Occultation experiments for the investigation of the vertical structure provide a vertical resolution much finer than the scale height, which is better than most other remote sensing or in-situ techniques on descent probes. Other than direct measurements by in-situ sensors on descent probes, the profiles sample a long refracted path through the atmosphere and local solar time may vary somewhat depending on the occultation geometry. Further, the coverage is not uniform due to reliance on the various occultation geometries - Sun-planet-spacecraft for SOIR, UV bright star-planet-spacecraft when it is on the night side of the planet for SPICAV and the Earth-planet-spacecraft for radio occultation - resulting in limited spatial coverage. The frequency of measurements and their locations of the profiles are largely determined by the spacecraft orbital parameters for all three methods

\subsubsection{Radio Occultations: Post Pioneer Venus and Pre-Venus Express Orbiter Missions}

The feasibility of the radio occultation technique to obtain atmospheric profiles of temperature and density with altitude was first demonstrated by Mariner 4 at Mars (Kliore et al. 1965) and then at Venus by Mariner 5 (Kliore et al. 1967) using both one way (spacecraft to Earth), and two way (Earth to spacecraft to Earth) occultations (Howard et al. 1974), generally at two frequencies (X and S band). Venera 9/10 occultations at $32 \mathrm{~cm}$ (Kolosov et al. 1978; Yakovlev et al. 1978) and 15/16 at 5 and $13 \mathrm{~cm}$ wavelengths (Yakovlev et al. 1987) orbiters also obtained radio occultation profiles by using these radio frequencies.

Until the Venus Express observations by SOIR and SPICAV, only the radio spectrum $(2.8,5,13,32 \mathrm{~cm}$ wavelengths) had been used for occultation retrievals of neutral atmosphere properties at Venus. SOIR used near infrared wavelengths for solar occultations (all latitudes, possible only at 6:00 AM and 6:00 PM local times) and SPICAV (all latitudes, night side only) ultraviolet wavelengths to retrieve the atmospheric properties. While both experiments - and VeRa in the radio wavelength regime - rely on atmospheric transmission, SOIR and SPICAV depend on spectral absorption to determine the $\mathrm{CO}_{2}$ density, and VeRa depends on the index of refraction. The temperature is then calculated using the hydrostatic law and assuming an atmospheric composition (for all three experiments). This may be a 
potential source of some small error $(<1 \mathrm{~K})$ above approximately $110 \mathrm{~km}$, where the atmospheric composition is known to have some variation as indicated by the homopause level from SOIR measurements (between 120-132 km according to Mahieux et al. 2012; Leovy 1982). The noise in the spectra and uncertainty in the HITRAN line intensities are the main sources of error (SNR 2000 for SOIR and 500-1000 for SPICAV).

We describe below a brief summary of the data collected by the three occultation experiments on the Venus Express orbiter, analysis approach and significant results.

\subsubsection{Temperature Profiles from Solar Occultations in the Infrared (SOIR)}

The SOIR instrument was an infrared spectrometer, which used the solar occultation technique to sound the mesosphere and the lower thermosphere of the Venus atmosphere (Mahieux et al. 2008; Nevejans et al. 2006). It was sensitive to the 2.29 to $4.43 \mu \mathrm{m}$ region (2257 to $4430 \mathrm{~cm}^{-1}$ ), and used an echelle grating at very high diffraction orders (from 101 to 194) to diffract the infrared sunlight. During an occultation, SOIR could measure up to four different orders every second, resulting in eight spectral sets downlinked to the Earth. All measurements always occurred at the terminator, i.e. the local solar time is either 6:00 AM or 6:00 PM, and all latitudes were well covered, except for the $30^{\circ}-60^{\circ} \mathrm{N}$ region due to the geometry of the spacecraft orbit. The vertical resolution, i.e. the vertical altitude range sounded by the projected slit at the limb of the atmosphere at the time of a measurement, varied from a few hundred meters for measurements at the North Pole to approximately $5 \mathrm{~km}$ when reaching the South Pole. The vertical sampling, i.e. the vertical distance between the mean altitude of two successive soundings, was also dependent on the latitude due to the geometry of the orbit, having values of approximately $2 \mathrm{~km}$ close to the North Pole, $\sim 500 \mathrm{~m}$ between $40^{\circ}$ and $70^{\circ}$ North, and rising up to $5 \mathrm{~km}$ close to the South Pole. The maximum altitude range probed by SOIR varied from $65 \mathrm{~km}$ up to $170 \mathrm{~km}$.

SOIR results on temperature profiles were obtained using two different approaches an iterative procedure to fit the observed spectrum from knowledge of the atmospheric constituents (Mahieux et al. 2010; Mahieux et al. 2012; Vandaele et al. 2015) and calculating the temperature directly from the structure of the rotational-vibrational bands (Mahieux et al. 2015b).

In the first approach, the $\mathrm{CO}_{2}$ number density profile is obtained first, and then the temperature profile is derived using the hydrostatic law. The resulting number density profile was used as a-priori for the next iteration, also using the new calculated temperature profile. The inversion was considered to have converged when both number density and temperature profiles were within the uncertainty of the previous step. The results of the inversion were the $\mathrm{CO}_{2}$ number density and temperature profile, and assuming a $\mathrm{CO}_{2}$ Volume Mixing Ratio (VMR) from a modified Venus International Reference Atmosphere (VIRA) from Hedin et al. (1983) and Zasova et al. (2006). The $\mathrm{CO}_{2}$ number density is shown in Fig. 11 (left panel). The corresponding temperature profiles (right hand-side panel, Fig. 11) show a large variability in the entire altitude range probed by SOIR (Mahieux et al. 2015a). However, a systematic structure is always observed, with a very cold layer around $120 \mathrm{~km}\left(10^{-5}\right.$ $\mathrm{mb}$, temperatures around $100 \mathrm{~K})$ surrounded by two warmer layers at $100 \mathrm{~km}\left(10^{-2} \mathrm{mb}\right.$, temperatures around $250 \mathrm{~K})$ and $150 \mathrm{~km}\left(10^{-7} \mathrm{mb}\right.$, temperatures around $\left.220 \mathrm{~K}\right)$.

Figure 12 shows the latitude dependence of the profiles at morning and evening terminators as a function of total pressure by combining the north and south hemispheres. The temperature inversion layers are seen to occur at all sampled latitudes. Figure 13 shows the mean vertical (pressure) profile of the $\mathrm{CO}_{2}$ number density (left) and temperature (right panel). 

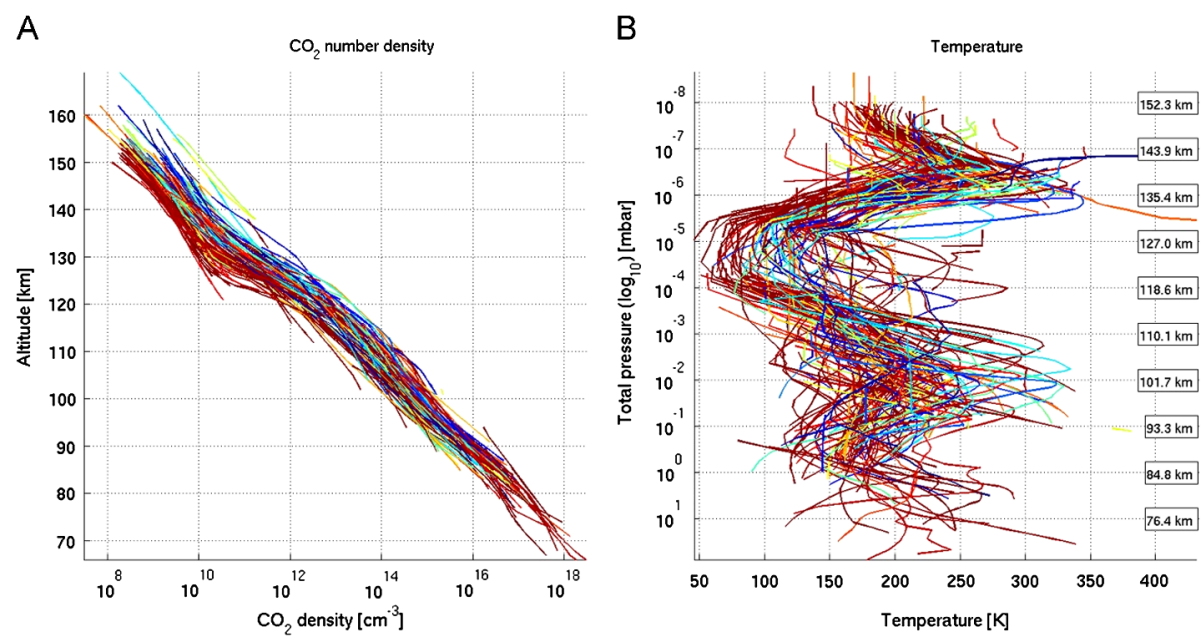

Fig. 11 (Left) All retrieved SOIR $\mathrm{CO}_{2}$ number density profiles (123). (Right) hydrostatic temperature profiles. In both panels colors represent absolute latitude: blue shades for equatorial observations and red shades for polar observations. (From Mahieux et al. 2015a)

A

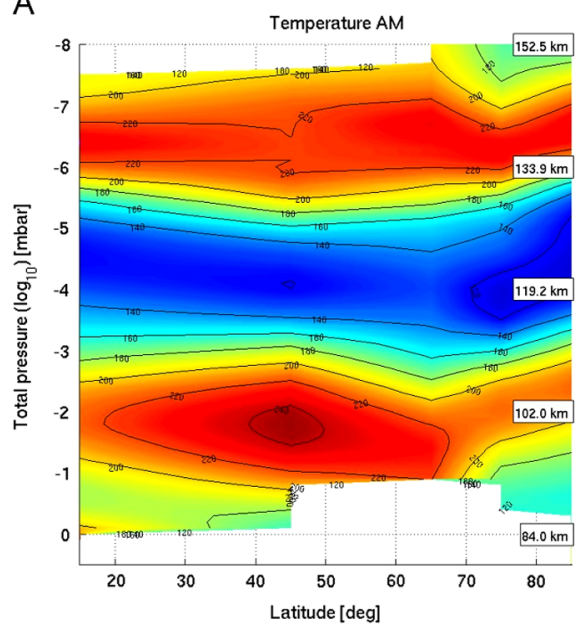

B

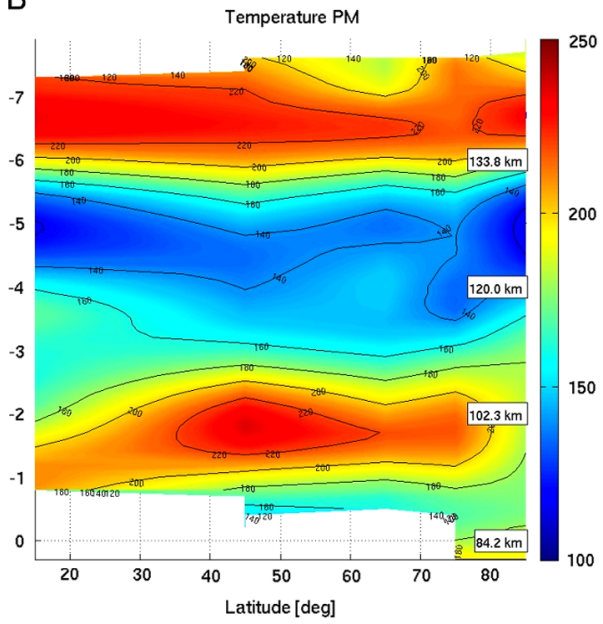

Fig. 12 Pressure-latitude cross section of SOIR temperatures calculated from the number density profiles for the morning side of the terminator (left) and for the evening side (right) from 1 to $10.8 \mathrm{mb}$. Approximate altitudes are indicated. North-South symmetry is assumed to increase the latitude sampling. (From Mahieux et al. 2015a)

The second method to infer the atmospheric structure is using the Mahieux et al. (2015b) presented profiles of temperature from the same SOIR data using the second method to calculate the temperature directly from the $\mathrm{SOIR} \mathrm{CO}_{2}$ spectra, by investigating the rotational structure of the rotational-vibrational bands, which could be resolved by the instrument. They describe how to account for all the instrument characteristics, in order to calculate the rotational temperature correctly from each spectrum. They showed that the method is reliable, and quick to derive the vertical temperature profile. However, the results show 

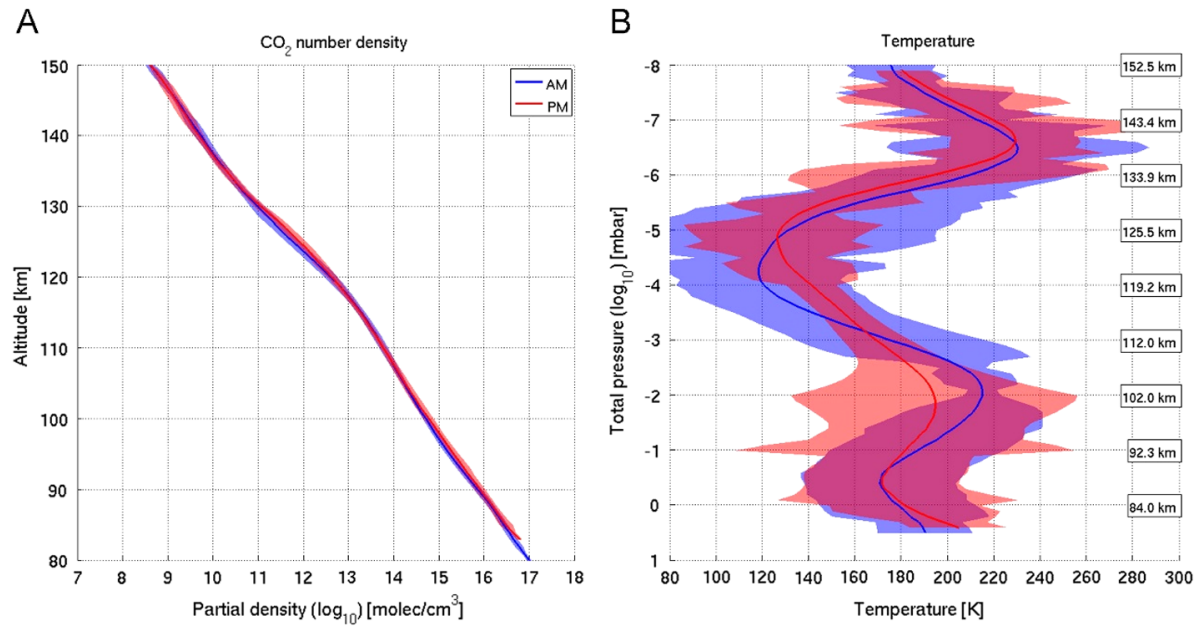

Fig. 13 Mean density (left panel) and mean temperature (right panel) as a function of the terminator side. The colored shapes represent the variabilities (standard deviation) for morning (blue) and evening terminator (orange). (From Mahieux et al. 2015a)

large uncertainties $(20-50 \mathrm{~K})$ due to the method itself, and are due to the uncertainty of the line intensities due to noise in the measured spectra according to Mahieux et al. (2015b). In contrast, the errors in the first method arise from ray tracing and deviations from the assumed homopause level in the retrieval procedure for a given profile. The study compares the mean value of the rotational temperature profiles with the general structure of the kinetic temperature profiles derived using the hydrostatic equilibrium (Mahieux et al. 2015a), within the very cold layer at $\sim 130 \mathrm{~km}$, see Fig. 14. In addition, no rotational non-LTE emissions have been observed.

\subsubsection{Temperature Structure from Radio Occultations - VeRa and Akatsuki Orbiters}

Only about twenty radio occultations were recorded from Magellan orbiter in October 1992 towards the end of its radar mission. The Venus Express orbiter has yielded more than 800 occultation profiles during 2006-2014 (Tellmann et al. 2012) and the Akatsuki orbiter is providing profiles since 2016 (Imamura et al. 2017). Whereas Venus Express used the radio subsystem of the spacecraft in Earth occultation geometry at two coherent radio frequencies (X- and S-band), Akatsuki orbiter radio occultations are obtained only using X-band. Both orbiters have yielded number density profiles of the neutral atmosphere $(\sim 40-90 \mathrm{~km}$ altitude), from the inferred refractivity of the atmosphere with vertical resolution of several hundred meters. Frequency stability from an onboard ultra stable oscillator in the one-way downlink transmission mode significantly improves the quality of the temperature profiles, which are deduced from the frequency shifts of the received signal due to the atmosphere. This was possible with Pioneer Venus, Magellan, Venus Express and Akatsuki orbiters but the Venera orbiters (Yakovlev et al. 1978, 1987) lacked such a frequency reference and the errors in the retrieved profiles are larger. Pressure and temperature profiles are derived from the density profiles assuming hydrostatic equilibrium and the ideal gas law. Details about the VeRa instrument are described by Häusler et al. (2006, 2007). The measurements cover nearly all local times, latitudes and longitudes with a gap in the northern middle latitudes resulting from the highly elliptical orbit of Venus Express. A deep temperature inversion 

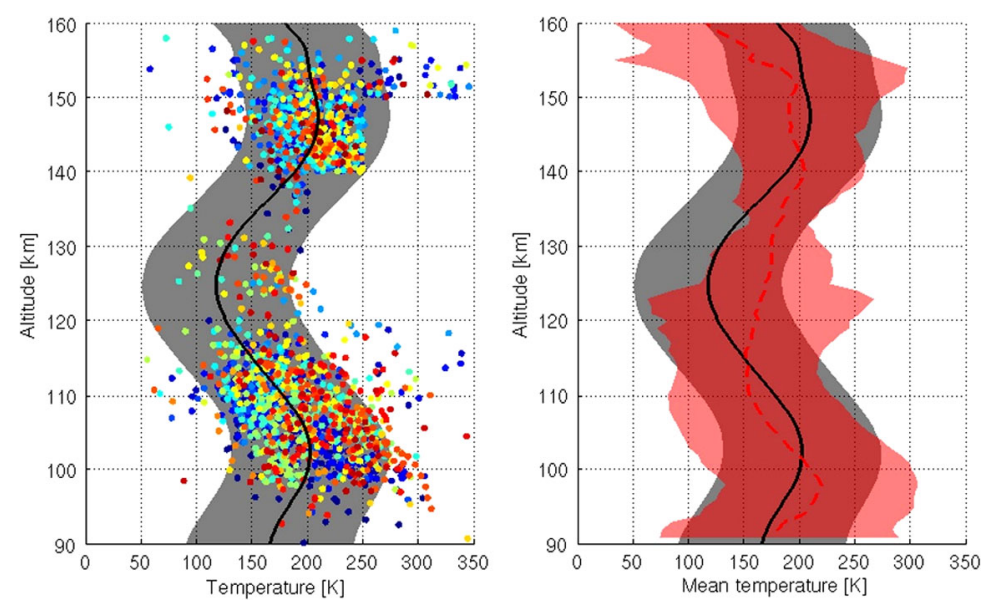

Fig. 14 Rotational temperature profiles calculated by Mahieux et al. (2015b). In the left panel, the derived rotational temperatures are shown (the error are not shown for clarity). The color code is the orbit number: blue for the first orbits, red for the last ones. The black profile and its envelope is the mean hydrostatic profile from all these orbits. In the right panel, the mean rotational temperature profile is presented as the red dashed curve, together with its uncertainty (reddish envelope)

was found in the high latitude region, in good agreement with former results. The atmosphere above the cold collar is characterized by an almost isothermal temperature field with embedded atmospheric waves displaying non-uniform scales (Fig. 15).

The low latitude profiles show only a decreasing temperature from all the profiles obtained thus far. Akatsuki occultation profiles of temperature are shown in Fig. 15 (tight). Tellmann et al. (2009) provided a more detailed analysis, from the VeRa data set obtained during the first three occultation seasons. Figure 16 shows a meridional cross section as a function of pressure constructed from all available VeRa profiles. The observed features support the findings from VIRTIS (Fig. 5). The main characteristics of the thermal structure and the temperature values are also in good agreement with those determined from the earlier radio occultation experiments (Kliore 1985) and the infrared radiometer experiment (Taylor et al. 1980) on the Pioneer Venus Orbiter. The temperature profiles at the 1 bar level show a strong latitudinal gradient of $\sim 30 \mathrm{~K}$ (equator-pole), confirming former results from the Pioneer Venus Orbiter Radio Science experiment (Kliore and Patel 1982). A distinct cold collar separates the troposphere from the mesosphere with an even stronger equator-pole temperature gradient in the troposphere and a pronounced reversed meridional temperature gradient in the mesosphere above an altitude corresponding to the $\sim 30$ mbar pressure level. The mesosphere is characterized by the presence of horizontally and vertically propagating atmospheric waves of different scales (planetary waves and gravity waves; Tellmann et al. 2012). The altitude structure of the thermal field is consistent with decaying winds in the upper mesosphere.

The static stability of the atmosphere, defined as the difference between the temperature lapse rate and the adiabatic lapse rate, also varies with altitude and latitude. The static stability is an important parameter for atmospheric dynamics. Positive values indicate a stably stratified atmosphere while negative values represent an atmosphere that is unstable towards convective overturning. Figure 17 shows typical examples of the static stability as a function of altitude derived from the VeRa temperature profiles. These profiles show a distinct latitudinal dependency with an extended region of low, near neutral stability in the middle cloud 

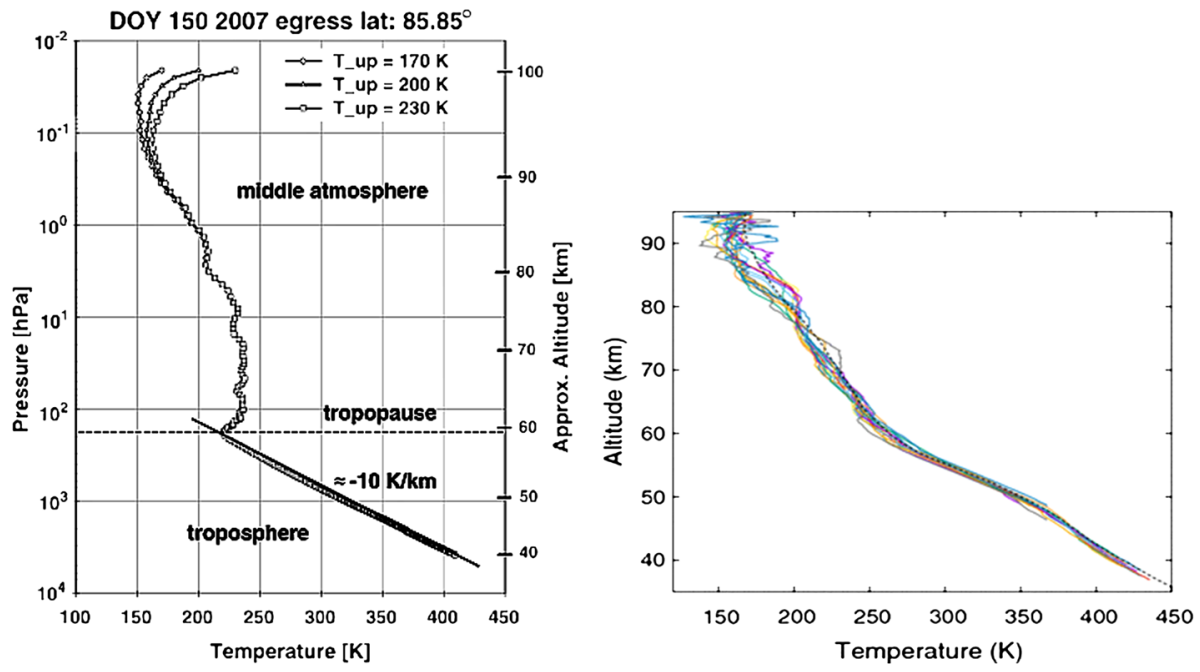

Fig. 15 (Left) $T[p(r)]$ at high latitudes (day of year (DOY) 150 2007). The occultation point at the 1-bar level (about $50 \mathrm{~km}$ ) corresponds to $8=85.85^{\circ} \mathrm{N}$, solar zenith angle is $89.5^{\circ}$, and local time (LT) is $1550 \mathrm{~h}$. Altitude is approximate relative to the mean Venus radius of $6051.8 \mathrm{~km}$ (Tellmann et al. 2009). Profiles are obtained with three initial temperature estimates at $100 \mathrm{~km}$ of 170, 200, and $230 \mathrm{~K}$ which quickly converge at an altitude near $90 \mathrm{~km}$ (about $0.3 \mathrm{hPa}$ ). Mean lapse rate $\sim 10 \mathrm{~K} \mathrm{~km}^{-1}$ is observed below the tropopause. The middle atmospheric lapse rate in the middle atmosphere is much lower; the temperature is nearl yisothermal up to the $10-\mathrm{hPa}$ level $(10 \mathrm{mbar}$ at roughly $75 \mathrm{~km})$. Small-scale fluctuations are often found in themiddle atmosphere. Temperature continues to decrease athigher altitudes up to the upper boundary of VeRa sensitivity near $0.02 \mathrm{hPa}(\sim 100 \mathrm{~km})$. On the right are shown some Akatsuki radio occultations (Imamura et al. 2017). The VIRA temperature profile for the low latitude $\left(<30^{\circ}\right)$ is also shown as a dashed curve for comparison. The radius of Venus is assumed to be $6051.8 \mathrm{~km}$

Fig. 16 Average temperature latitude-pressure cross section, as derived from all available VeRa profiles and assuming hemispheric symmetry. The tropopause temperatures are between $260-280 \mathrm{~K}$ in low latitudes and decrease to between $220-230 \mathrm{~K}$ at $70^{\circ}$ latitude and increase to about $240 \mathrm{~K}$ at the poles (Tellmann et al. 2009)

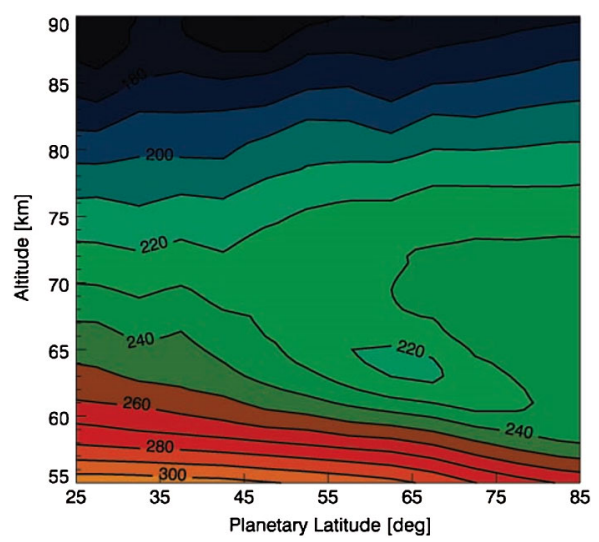

layer at medium/high latitudes followed by a highly stable atmosphere at higher altitudes. The equatorial region, on the other side, is characterized by only shallow layers of low static stability (Fig. 17). These regions of low, near neutral, stability are likely convective layers because they enable convective turnover of fluid parcels due to low restoring force. This might give rise to the generation of vertically propagating gravity waves responsible for the 

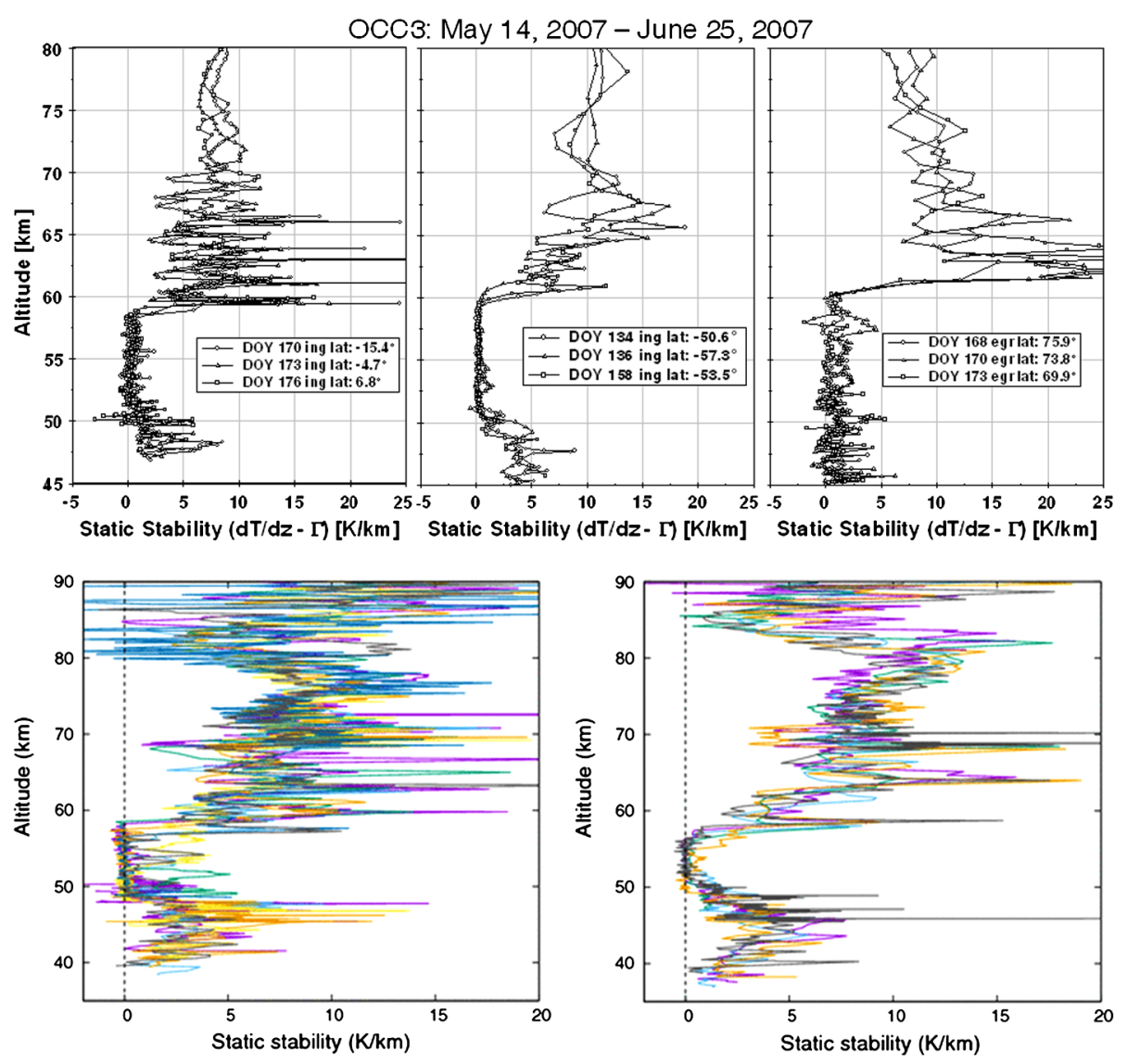

Fig. 17 (Top) a. Profiles of static stability for different latitude regions. Left: low latitudes, middle: mid-latitudes, right: high latitudes. From Tellmann et al. (2009). (Bottom) For comparison, some static stability profiles calculated for some Akatsuki radio occultation temperature profiles in the latitude of $40^{\circ} \mathrm{S}-40^{\circ} \mathrm{N}$ on the (upper) morning side with local times of 01:10-07:10 and (lower) afternoon side with local times of 16:10-17:30 (from Imamura et al. 2017)

transport of momentum, which is a key ingredient for understanding the superrotation of the Venus atmosphere.

The VeRa data analysis was performed from the signal obtained with the ground receiver operating in the closed loop mode. When operating in the open loop mode however, the ground receiver can additionally resolve temperature variations confined to very small altitude intervals as typical for multipath effects (radio frequency propagation effects caused most likely by atmospheric density irregularities). Effects of this kind were observed in a small confined altitude region in the cloud layer within an altitude interval of $\sim 1 \mathrm{~km}$ poleward of $60^{\circ}$ latitude in both hemispheres and are characterized by negative-positive temperature excursions, which can reach $5 \mathrm{~K}$ to $10 \mathrm{~K}$ (Herrmann et al. 2015).

The cold collar (mentioned above), observed in both hemispheres, is correlated with a drop of the tropopause (defined by Kliore 1985 as lapse rate $<8 \mathrm{~K} \mathrm{~km}^{-1}$ ) altitude by $\sim 7 \mathrm{~km}$ $(60 \mathrm{~K})$ when approaching the polar regions from the equator (Tellmann et al. 2009). This drop in the tropopause altitude is correlated with a decrease in the Venus cloud top (Fig. 18) 


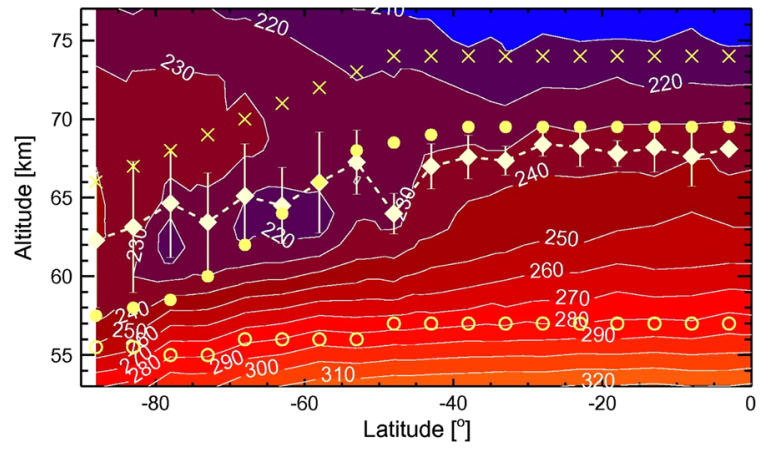

Fig. 18 Cloud top altitude as a function of latitude overlaid on the VeRa temperature field. The different symbols mark different studies of the cloud top altitude: diamonds - study by Lee et al. (2012) at 4-5 $\mu \mathrm{m}$; crosses - VIRITS spectroscopy in the $1.6 \mu \mathrm{m} \mathrm{CO} 2$ band (Ignatiev et al. 2009); filled and open circles approximate Venera-15 Fourier spectroscopy in the 8.2 and $27.4 \mu \mathrm{m}$, respectively (Zasova et al. 2007b). (From Lee et al. 2012.) See Titov et al. (2018) for an update to this figure

Fig. 19 Small-scale temperature fluctuations (vertical wavelengths $1-4 \mathrm{~km})$ as a function of latitude and altitude. From Tellmann et al. (2012)

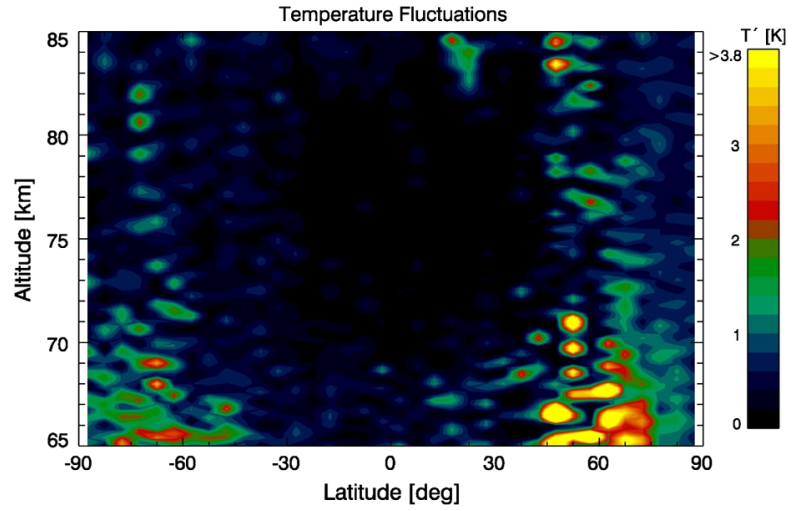

as investigated by Lee et al. (2012), using VeRa and VIRTIS data. From their analysis, the cloud top decreases from the low latitudes to the pole by $5 \mathrm{~km}$, i.e. from $\sim 67.2 \mathrm{~km}$ to $62.8 \mathrm{~km}$ altitude. The vertical spacing of the isotherms increases from low to high altitudes at all latitudes, but more so at polar latitudes, indicating the influence of clouds on the thermal structure. The deep temperature inversions in the cold collar region coincide with the cloud top position indicating the importance of radiative cooling in this altitude region and/or anomalous heat transport driven by eddies.

The high vertical resolution of the VeRa profiles provides the opportunity to detect small scale vertical wave structures. Tellmann et al. (2012) presented a global analysis of gravity waves with vertical wavelengths of $4 \mathrm{~km}$ or shorter (Fig. 19). The wave activity shows a strong altitude dependence with only very shallow waves in the adiabatic middle cloud region and high wave amplitudes in the adjacent upper cloud layer $(\geq 65 \mathrm{~km})$. The wave amplitudes in the mesosphere decrease with increasing altitude mainly due to radiative damping. This finding is in good agreement with former results from Magellan (Hinson and Jenkins 1995). Ando et al. (2016) find that the spectrum of gravity waves tends to follow the semi-empirical spectrum of saturated gravity waves, suggesting that the gravity waves are dissipated by saturation as well as radiative damping. The associated diffusive processes can play an important role in the transport processes for energy and momentum in the atmo- 
Fig. 20 Latitude-height cross section of Richardson number. The dotted line shows approximate level of cloud tops. (From Piccialli et al. 2012)

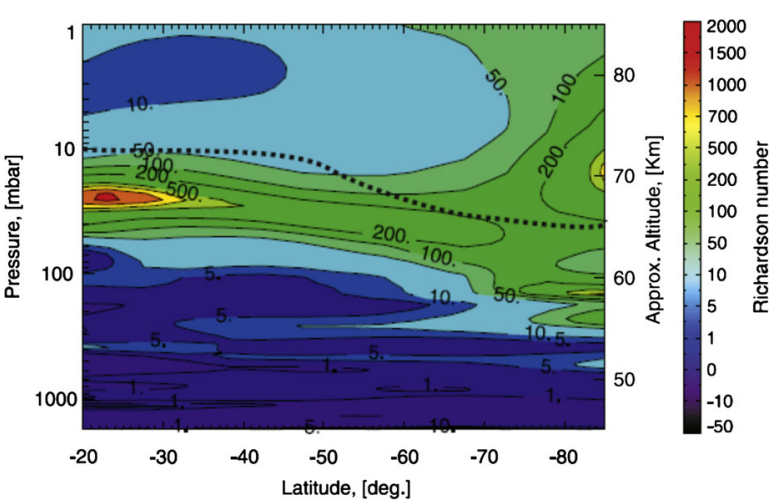

sphere (Ando et al. 2016). The wave activity also shows a distinct latitudinal dependence, with the highest wave amplitudes in the high latitude regions of both hemispheres (Fig. 19). The wave amplitudes in the northern hemisphere are slightly higher than those in the south, with the highest values west (downstream) of Ishtar Terra, the highest elevation on Venus, thus indicating that the interaction of the planetary surface with winds might be the driving source of wave activity. Topographical connections of these atmospheric waves were first reported from the measurements of wind from VeGa balloons (Young et al. 1987) at low equatorial latitudes and detected at high northern latitudes from Venus Express Venus Monitoring Camera (VMC) data by Piccialli et al. (2014), by analyzing cloud features from VMC observations (in northern high latitudes). Bertaux et al. (2016) also report on the influence of the topography on stationary gravity waves seen in VMC UV images at the cloud top level leading to a variation of the zonal wind speed above Aphrodite Terra.

Tellmann et al. (2012) showed that gravity waves in the equatorial region also exhibit a moderate local time dependency suggesting that convection in the middle cloud layer might contribute to the observed wave activity. The high wave activity in the high latitude region might therefore also be correlated with the deep convective clouds and the extended low stability region (Tellmann et al. 2009) as shown in the top right panel of Fig. 17.

The strong mesospheric latitudinal temperature gradient is correlated with strong zonal jets in the mesosphere of Venus. Piccialli et al. (2012) used VeRa profiles to infer the zonal wind vertical structure with latitude on Venus by assuming cyclostrophic balance in the Venus atmosphere directly from the pressure topography (Limaye 1985) and by thermal wind (which requires a knowledge of the wind at a boundary) by Newman et al. (1984). The VeRa results were found to be in good agreement with these former results and also with the Venera 15 Fourier Spectrometer (Schäfer et al. 1990; Zasova et al. 1999) and Venus Express cloud tracking results (Khatuntsev et al. 2014). Both the derived wind fields and the temperature structure were used to study the stability of the atmosphere with respect to turbulence and convection by Piccialli et al. (2012). A low Richardson number was found in the altitude range between $\sim 45$ and $\sim 60 \mathrm{~km}$ at all latitudes, corresponding to the lower and middle cloud layer and indicating a possible presence of turbulence as shown in Fig. 20. SánchezLavega et al. (2017) present a more detailed discussion of the turbulence, barotropic and baroclinic instability in the Venus atmosphere.

Tellmann et al. (2012) have investigated the local time dependency of the VeRa temperature field. A pronounced semidiurnal tidal structure was found in the equatorial region ( $\pm 20^{\circ}$ latitude) with its highest amplitudes in the upper mesosphere (Fig. 21). The observed structure is indicative of an upward propagating wave, supporting former results from the 
Fig. 21 Thermal structure as a function of local time and pressure in the equatorial region (lat $\leq \pm 20^{\circ}$ ). The thermal structure is approximated by a diurnal mean temperature and the first two tidal modes.

A pronounced semidiurnal structure develops in the upper mesosphere around the morning and evening terminators

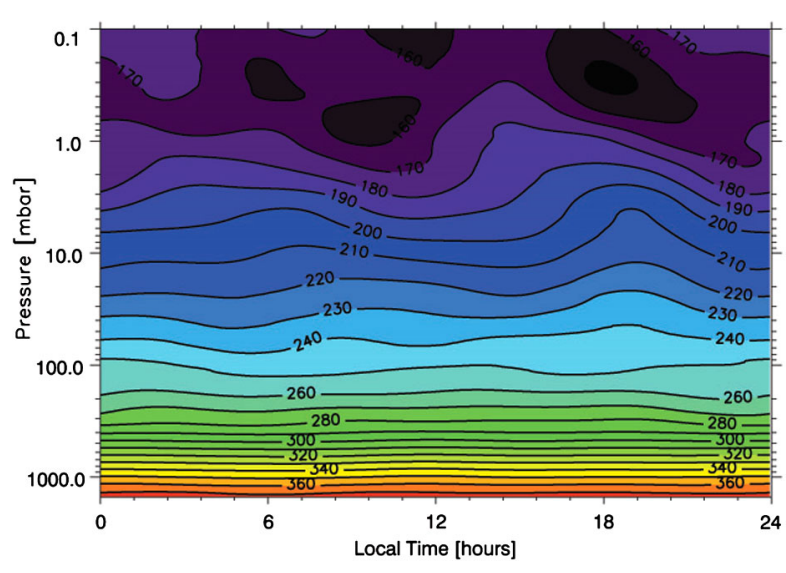

OIR experiment on the Pioneer Venus Orbiter (Schofield and Taylor 1983). Sánchez-Lavega et al. (2017) provide a comprehensive review of the role of thermal tides in the Venus atmospheric circulation.

\subsubsection{Temperature Profiles from Ultraviolet Stellar Occultations (SPICAV)}

The SPICAV instrument operated on board the European Venus Express spacecraft for eight years, beginning in 2006 (Bertaux et al. 2007a). The remote sensing spectrometer covered spectral regions in the ultraviolet (110 to $320 \mathrm{~nm}$ ) and in the near-infrared (1000 to $1700 \mathrm{~nm}$ ). SPICAV worked in three different geometries, nadir, solar and stellar occultation. In the stellar occultation mode, the ultraviolet channel was particularly well suited to measure the vertical profiles of $\mathrm{CO}_{2}$ local density, temperature, $\mathrm{SO}_{2}, \mathrm{SO}$, clouds and aerosols of Venus upper atmosphere from 90 to $140 \mathrm{~km}$ (Bertaux et al. 2007b; Montmessin et al. 2011).

During December 2006 and February 2013, 587 stellar occultations were acquired by SPICAV and analyzed (Piccialli et al. 2015). The observations covered all latitudes on the nightside (6:00 pm to 6:00 am local solar time). The vertical resolution of a profile ranges from 500 meters to $\sim 7 \mathrm{~km}$. The main features observed in the temperature structure are: (i) a permanent layer of warm air at 90-100 km altitude, and (ii) a constant decrease of temperature with altitude reaching minimum values of $\sim 100-130 \mathrm{~K}$ above $120 \mathrm{~km}$. In good agreement with previous observations (Mahieux et al. 2015a; Migliorini et al. 2012), SPICAV thermal structure exhibits a symmetry in terms of latitude between the two hemispheres. Local time variations dominate the structure of the Venus atmosphere at these altitudes: temperatures show an increase of about $20 \mathrm{~K}$ on the morning side as compared to the evening side. Moreover, a significant variability both on day-to-day as well as longer timescales affects the thermal structure of the Venus upper atmosphere. Temperatures can display variations of $\sim 10 \mathrm{~K}$ on timescales of $24 \mathrm{~h}$ and up to $\sim 50 \mathrm{~K}$ on timescales of a few (Earth) months. The $\mathrm{CO}_{2}$ homopause altitude was also determined; it varies between 119 and $138 \mathrm{~km}$ of altitude, and exhibits a high variability. The altitude shows a strong dependence on the local time, increasing from the evening side to the morning side. Figure shows a local solar time-altitude (top) and solar time-pressure (bottom) cross sections generated from SPICAV results (Piccialli et al. 2015). The spatial sampling of the profiles is somewhat sparse compared to other experiments (Limaye et al. 2017) and is significantly better between $25-30^{\circ}$ latitude bin combining north and south hemispheres. A weak dependence on local time is seen with somewhat warmer temperatures at 4 am above $90 \mathrm{~km}$ and 
Fig. 22 (Top) Temperature variation with local time and altitude and with latitude and altitude cross section from SPICAV temperature profiles. The latitudinal sampling from the SPICAV occultations is not uniform as can be seen in the sampling shown in the bottom panel. 587 profiles are used in generating these cross sections. (From Piccialli et al. 2015
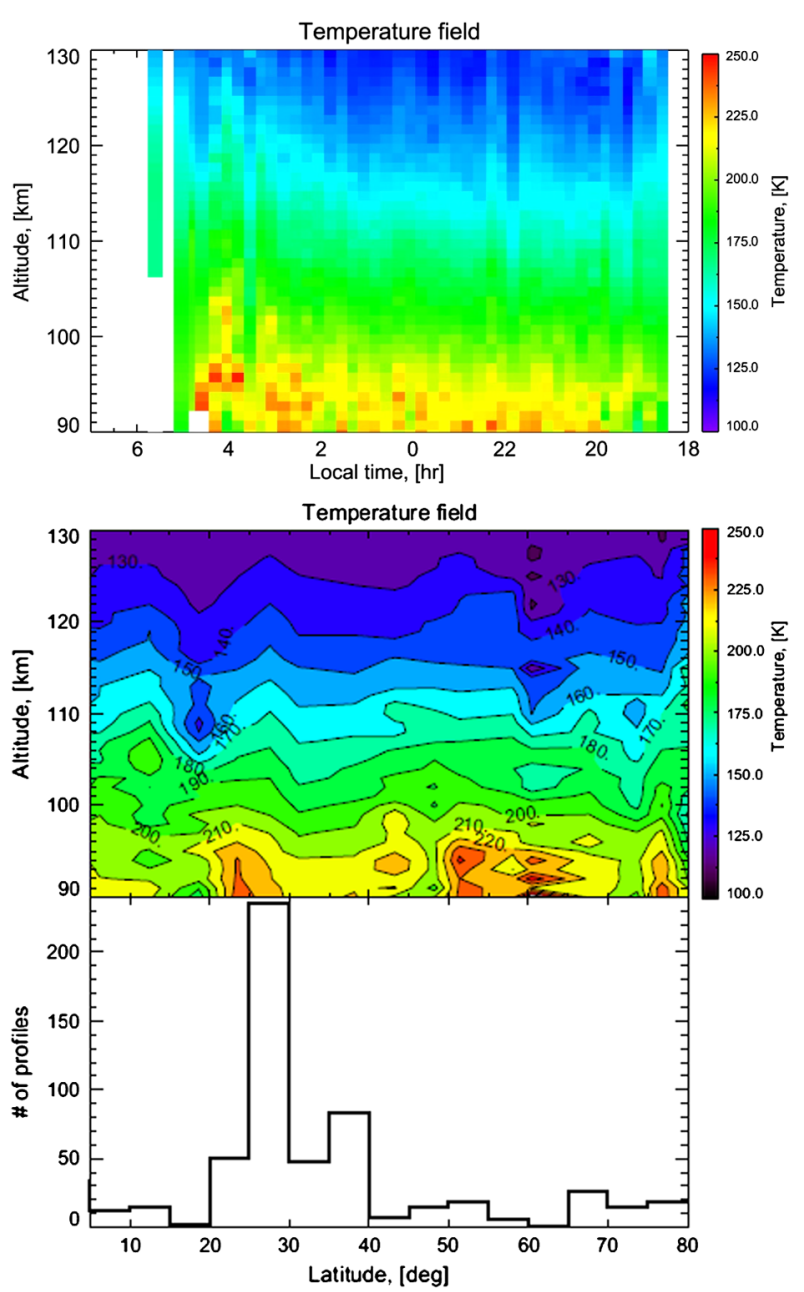

cooler temperatures around midnight above $115 \mathrm{~km}$. Piccialli et al. (2015) also present static stability profiles, which show that the stability increases with altitude above $100 \mathrm{~km}$ at all latitudes and also increases with decreasing altitude below $100 \mathrm{~km}$ in low and high latitudes.

\subsection{Ground Based Observations of Temperature Structure}

Temperature profiles above the clouds tops $(\sim 65 \mathrm{~km})$ to $120 \mathrm{~km}$ obtained by spectroscopy at the sub-millimeter wavelengths $(335-346 \mathrm{GHz})$ have been obtained in the last decade from the Atacama Large Millimeter Array (ALMA) during November 2011 (Piccialli et al. 2017) and from the James Clark Maxwell Telescope (Clancy et al. 2012). Previously Encrenaz et al. (1995) obtained a value of $140 \pm 10 \mathrm{~K}$ at $95 \mathrm{~km}$ from disk integrated measurements at $183 \mathrm{GHz}$. A latitudinal profile of temperatures at $110 \mathrm{~km}$ altitude was obtained by Sonnabend et al. (2008) using the non-thermal emission lines in the $10.6 \mu \mathrm{m}$ band of $\mathrm{CO}_{2}$ with values between $225-255 \mathrm{~K}$ at the equator and decreasing to between $160-170 \mathrm{~K}$ at $\pm 70^{\circ}$ latitudes at local noon. 
All of these investigations provide evidence of temporal/local time variability at the higher altitudes. Generally, these observations have large spatial foot-prints on the planet and the temporal coverage is sporadic but they facilitate comparing concurrent spacecraft data.

Finally, because of the rarity and uniqueness, the results of some inferences about upper atmosphere thermal structure from the analysis of the aureole observations during the transit of Venus across the solar observed from spacecraft and ground based telescopes are also worth mentioning (Peralta et al. 2016; Tanga et al. 2012) as analog for atmospheres of terrestrial exoplanets. More details of the ground based observations can be found in the intercomparison of recent observations reported by Limaye et al. (2017).

\section{Key Aspects of the Vertical Structure}

The vertical structure of the Venus atmosphere is influenced by the global cloud cover, the atmospheric composition and the global circulation. It is characterized by latitudinal gradients of temperature and by static stability variations. As presented in Sect. 3, the meridional gradients of temperature are small below about $60 \mathrm{~km}$, with decreasing temperatures from equator to the poles consistent with the zonal flow in approximate cyclostrophic balance (Leovy 1973). Above about $70 \mathrm{~km}(\sim 5 \mathrm{mb})$, the temperatures increase towards the poles on constant altitude levels leading to a breakdown of the cyclostrophic balance. This reversed gradient persists until about $90 \mathrm{~km}$ and then at higher altitudes changes sign again. The most dramatic change in the static stability takes place near the base of the cloud layer when the stability increases with altitude $(\sim 50 \mathrm{~km})$ whereas the lower atmosphere is almost adiabatic or even slightly unstable. Another change in the stability is in the "cold collar" region between about $60-70^{\circ}$ north and south latitudes where the coldest temperatures are found at about $67 \mathrm{~km}$ and a nearly isothermal layer exists. Surprisingly there appears to be no significant signature of the cold collar in the cloud top altitude (Ignatiev et al. 2009; Fedorova et al. 2016) at least in the near infrared data.

Lacking a stratosphere, the tropopause on Venus was first defined by Kliore (1985) as the level where the temperature lapse rate exceeds $-8.0 \mathrm{~K} \mathrm{~km}^{-1}$, with the troposphere extending to the surface below. Temperature inversions are generally seen in occultation profiles at mid to polar latitudes with low latitude profiles showing regions of increasing static stability. While the tropopause in higher latitudes is clearly marked by a sudden change in the temperature lapse rate, the identification of a tropopause in the equatorial region requires an explicit definition due to the lack of a pronounced temperature minimum. The tropopause level $(\sim 58 \mathrm{~km}$ in equator and polar latitudes and $\sim 62 \mathrm{~km}$ for cold collar) at all latitudes (Tellmann et al. 2009) is below the observed cloud tops the cloud tops which are estimated to be at $74 \pm 1 \mathrm{~km}$ in equatorial latitudes and between 64-69 km in polar latitudes (Ignatiev et al. 2009). The atmosphere below $\sim 62 \mathrm{~km}$ at all latitudes is characterized by a monotonically decreasing temperature from a surface value of $\sim 735 \mathrm{~K}$ and a surface pressure of $\sim 95$ bars, to values of $\sim 245 \mathrm{~K}( \pm 35 \mathrm{~K})$ at the $200 \mathrm{mb}$ level (altitude 58-63 km) (Kliore and Patel 1982; Tellmann et al. 2009). The vertical temperature gradient in the troposphere is strongly influenced by the presence of the different cloud layers. The major part of the incoming solar radiation that is not reflected back by the highly reflective cloud cover is absorbed in the cloud region where the clouds heat the regions well above and below their boundaries. Changes in the temperature lapse rate with altitude in the troposphere coincide with the boundaries of the cloud layers (Blamont and Ragent 1979). The troposphere is generally stable with 
confined regions of neutral or very low static stability near the base of the cloud layer and below $20 \mathrm{~km}$.

Above the tropopause is the mesosphere, which is highly stable and characterized by a strong variability over time as well as solar zenith angle. The lower mesosphere (70$90 \mathrm{~km}$ ) is characterized by the reversed meridional temperature gradient (compared to lower levels), decreasing temperatures with altitude, and increasing temperature variability. The upper mesosphere $(90-150 \mathrm{~km})$ shows a more complex structure with alternating warm and cool layers and very cold temperatures, reaching $120 \mathrm{~K}$. We present below some additional details of the structure in different altitude layers from surface to $90 \mathrm{~km}$ and higher.

\subsection{Surface to $45 \mathrm{~km}$ - Entry Probes and Overlapping Radio Occultations}

Although there have been no new measurements of the deep atmospheric temperature structure since the VeGa 2 lander measurements (Linkin et al. 1986a), it is worth visiting the lower atmosphere thermal structure to draw attention to some aspects which are not yet well understood. Closed loop radio occultation profiles generally provide temperatures down to about $40-45 \mathrm{~km}$, but open loop data can provide information somewhat deeper, down to perhaps the $35 \mathrm{~km}$ altitude level. The bending of the radio beam in the deep atmosphere below about $32 \mathrm{~km}$ due to refraction prevents determination of the thermal profile down to the surface. Thus, the thermal structure in the 35 to the surface can be measured with high vertical resolution only by in-situ measurements.

Meadows and Crisp (1996) estimated the stability in the $6 \mathrm{~km}$ layer (difference between the adiabatic lapse rate and the ambient temperature lapse rate) from surface to $6 \mathrm{~km}$ altitude from near infrared observations from Earth based telescopes using the spectral windows at 1.0, 1.1 and $1.1 \mu \mathrm{m}$ to estimate the water vapor abundance. Their results suggest a stable atmosphere in contrast to the VeGa 2 results discussed below.

Our current knowledge about this region is therefore based on a very small number of profiles from the Venera (Marov et al. 1973; Avduevsky et al. 1976, 1979) and Pioneer Venus probes (Seiff et al. 1980) to $12 \mathrm{~km}$ altitude and only one profile below $12 \mathrm{~km}$ (VeGa 2 lander). A detailed description of the deep Venus atmosphere can be found in Seiff (1983). Altitude values in the deep atmosphere were inferred from the pressure and temperature values, assuming hydrostatic equilibrium. To remove topographical differences, all data from different probes have been adjusted to a common landing elevation (Seiff 1983), so care should be taken to compare other profiles for accurate comparison of temperature vs altitude.

Due to lack of actual measurements below about $12 \mathrm{~km}$ from the Pioneer Venus probes, temperature values were extrapolated downwards to the mean surface using the adiabatic lapse rate (Seiff et al. 1980). Subsequently the VeGa 2 lander returned lower atmospheric temperature data (Linkin et al. 1986a) which reveal a slightly different lapse rate. Additionally, temperature data from the SNFR instrument (Sromovsky et al. 1985) were compared with the Atmospheric Structure experiment on Pioneer Venus (Seiff et al. 1980) which provide additional information about the errors and confirmation of the general structure above $12 \mathrm{~km}$ altitude.

Low static stability regions were detected by the probes and by remote sensing between 20 and $30 \mathrm{~km}$, and below $10 \mathrm{~km}$ and in the middle cloud layer (Seiff 1983). Diurnal changes in the deep atmosphere are expected to be very low $(<1 \mathrm{~K})$ due to the large thermal inertia of the thick atmosphere. However, such high accuracy measurements have yet to be made at most latitudes and at different local times. In the stable layers, differences between the Pioneer Venus Day and Night probes indicate oscillatory temperature fluctuations, possibly caused by planetary scale waves including solar thermal tides. 
Since the early measurements of the high temperature and pressure on the surface of Venus, it has been known that the ideal gas law cannot be used for certain calculations involving the equation of state but that the real gas equation has to be used (Staley 1970). The critical point properties for $\mathrm{CO}_{2}$ and $\mathrm{N}_{2}$ have been known for a long time. Thus it has been known that under the temperature and pressure conditions near the surface of Venus, both the primary constituents should be in supercritical state. Yet, surprisingly little attention has been paid to the effects of the mixture of two supercritical fluid states of the primary constituents in the lower atmosphere of Venus, and to the observation that a vertical gradient in the abundance of nitrogen was measured by the Pioneer Venus Large Probe GCMS (Oyama et al. 1980) which could not be explained. Recent laboratory experimental data suggest that homogenous mixtures of supercritical carbon dioxide and nitrogen tend to separate vertically when left alone (Hendry et al. 2013).

Seiff et al. (1980) noted the need for real gas equation of state for the calculation, first pointed out by Staley (1970), but approximated the impact of 3.5\% nitrogen and computed the adiabatic lapse rate using the relationship (Staley 1970):

$$
\Gamma=-(\alpha T) g / C_{p} \quad \text { and } \quad \alpha=-(\delta \rho / \delta T) / \rho
$$

where $\alpha$ is the compressibility, $T$ is the atmospheric temperature, $g$ is acceleration due to gravity for Venus (function of altitude), $C_{p}$ is the specific heat, and $\rho$ is the atmospheric density. $\alpha$ is unity for an ideal gas, but varies with pressure and temperature, as does $C_{p}$. Seiff et al. used tables of $C_{p}$ and $\alpha$ compiled from experimental data by Hilsenrath et al. (1960). Dutt and Limaye (2018) used the GERG 2008 (Kunz and Wagner 2012) model for the real gas equation of state for a binary mixture and obtained values of the adiabatic lapse rate for the conditions found in the Venus atmosphere by calculating the values of $C_{p}$ from the equations of state for the two real gases. The interactions between the two gases were included in the calculation. Calculations show that the $100 \% \mathrm{CO}_{2}$ approximation made by Seiff et al. (1980) is reasonable considering low abundance of nitrogen as can be seen from Fig. 23 which shows the difference between the VIRA values (Seiff et al. 1985) for the adiabatic lapse rate and the results of Dutt and Limaye (2018). Figure 24 shows how the adiabatic lapse rate varies with altitude using the $\mathrm{VeGa} 2$ lander profile of temperature (Linkin et al. 1986a).

The VeGa 2 lander is the only probe to have returned temperature data below $12 \mathrm{~km}$ (Fig. 24). These data indicate that at the surface, the lapse rate appears to be somewhat stable, but a thin super-adiabatic layer is centered at about $4 \mathrm{~km}$ above the surface, perhaps the most interesting result regarding the atmospheric thermal structure in the near surface region. The existence of the unstable layer above the surface is puzzling as the lapse rate at the surface is stable, unlike the case on Earth where such near surface unstable conditions are encountered over asphalt or deserts. Above $8 \mathrm{~km}$, the atmosphere becomes increasingly stable with peak stability at about $15 \mathrm{~km}$ and becoming unstable in a thin layer between 19$20 \mathrm{~km}$ and becoming weakly stable above $20 \mathrm{~km}$ to about $30 \mathrm{~km}$. It is puzzling how such unstable/stable layers can be caused by radiative effects from a clear atmosphere alone. The presence of a near surface aerosol layer has been suggested by Venera probe measurements (Grieger et al. 2004) but little is known about their radiative effects.

Lebonnois and Schubert (2017) considered the lower atmospheric stability profile in terms of the lapse rate of potential temperature and proposed that that this unstable region arises due to the assumed nitrogen abundance due to density separation of the $\mathrm{CO}_{2}$ and $\mathrm{N}_{2}$ which are both in supercritical state near the surface. They propose that if the nitrogen abundance decreases to zero at the surface, the resulting profile then is stable. 
Fig. 23 Differences in the dry adiabatic lapse rates computed for VIRA profile using different real gas equations of state (GERG 2008) and VIRA Model approximation for a $96.5 \%$ $\mathrm{CO}_{2}+3.5 \% \mathrm{~N}_{2}$ mixture (Dutt and Limaye 2018) and compared with pure $\mathrm{CO}_{2}$
Fig. 24 Static stability of the VeGa 2 lander temperature profile calculated using the adiabatic lapse rate from the real gas equation of state (Dutt and Limaye 2018) for $\mathrm{CO}_{2}+\mathrm{N}_{2}$ mixture confirms the unstable layer below $7 \mathrm{~km}$ (shown in purple). The VIRA deep atmosphere adiabatic lapse rate (Tables 1.1 and 1.2 from Seiff et al. 1985) is also shown (orange)
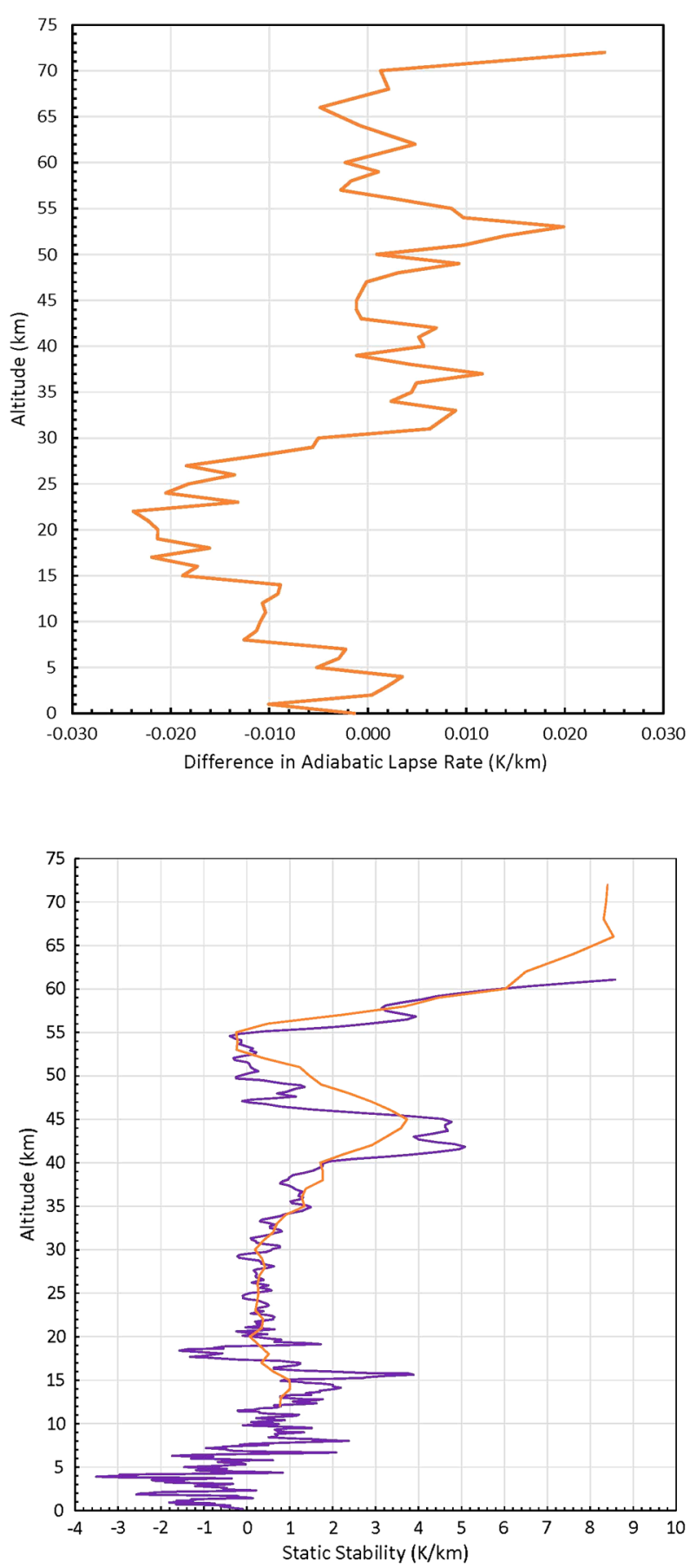

This is consistent with the inference of Meadows and Crisp (1996) of a stable layer in the lowest $6 \mathrm{~km}$ of the atmosphere, also on the night side. Whether this comparison between the 

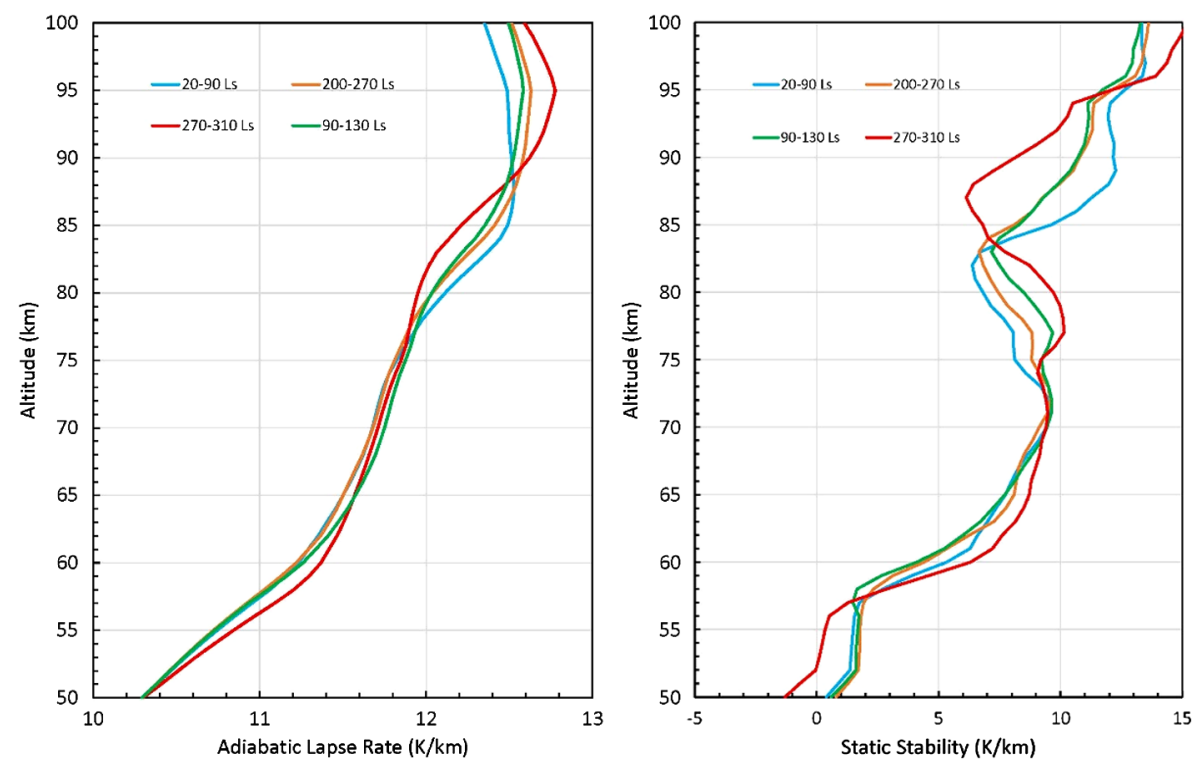

Fig. 25 Lapse rates (left) and static stability profiles (right panel) for the temperature profiles retrieved from the Venera 15 Fourier Spectrometer data in four solar longitude quadrants (Zasova et al. 2006) using the real gas mixture adiabatic lapse rates (Dutt and Limaye 2018)

large area covered by the Meadows and Crisp observations and the point measurements from $\mathrm{VeGa} 2$ lander is valid can only be ascertained by future in-situ and telescope observations.

Despite the progress in improving the equation of state for real gas mixtures, which enable the specific heat of real gas mixtures at constant pressure to be calculated reliably instead of being measured experimentally, there are some discrepancies in estimating densities (Goos et al. 2011).

The consequences of the mixture of two real gas supercritical fluids was not recognized until experimental results of $\mathrm{CO}_{2}+\mathrm{N}_{2}$ mixtures under supercritical state showed that density separation occurs when left alone in a chamber (Hendry et al. 2013). It is critical to verify therefore, whether such a separation occurs in a natural state on Venus, and validate the results by laboratory measurements. The potential impact of such density separation on the temperature profiles obtained to date from spacecraft data is discussed in Sect. 6.

Figure 25 compares the variation of adiabatic lapse rates and static stability with local time for four local time quadrants $\left(L_{s}\right)$ for which average profiles were generated by Zasova et al. (1999) from Venera 15 Fourier Spectrometer data. Large differences $\left(\sim 5 \mathrm{~K} \mathrm{~km}^{-1}\right)$ in static stability are seen in a layer centered at about $87 \mathrm{~km}$ altitude in the four quadrants. A sharp decrease in static stability is noticeable below $70 \mathrm{~km}$ in all quadrants and becoming near neutral below $\sim 55 \mathrm{~km}$ in the $L_{s} 270-310^{\circ}$ quadrant.

\section{$4.245 \mathrm{~km}$ to $60 \mathrm{~km}$}

Due to opacity of the clouds, most of the information on the region between 45 and $60 \mathrm{~km}$ comes from in situ measurements by entry probes or radio occultations. Retrievals from remote infrared observations down to $55 \mathrm{~km}$ were presented by Zasova et al. (1999), and Haus et al. (2014), and relied on simultaneous retrieval of aerosol opacities, as well as by GarateLopez et al. (2015) who exploited the favorable conditions of very low cloud altitudes over 
the south polar dipole. The vertical temperature gradient in the middle cloud region usually lies very close to the adiabatic value (Tellmann et al. 2009), with an approximate value of $10 \mathrm{~K} \mathrm{~km}^{-1}$. The depth of this neutral stability region shows a moderate latitudinal dependency with the deepest layers located at high latitudes and very shallow layers in the equatorial region (Tellmann et al. 2009). Above the middle cloud, the vertical temperature gradient becomes much weaker and departs from the adiabatic value at altitudes between 57 and $63 \mathrm{~km}$ (Kliore 1985; Tellmann et al. 2009). This level represents the effective tropopause on Venus and marks the beginning of the mesosphere, where the atmosphere is statically stable and highly variable. In the VeRa data, the tropopause appears to occur at higher altitudes $(63 \mathrm{~km})$ close to the cold collar and much lower in the equatorial region $(58 \mathrm{~km})$ and at the poles. This trend is also seen in the Pioneer Venus (Kliore 1985), and Magellan radio occultation results (Jenkins et al. 1994). The upper parts of the troposphere immediately below the tropopause present a clear temperature latitudinal gradient with the atmosphere being 30 $\mathrm{K}$ warmer at the equator than at the pole (Fig. 16).

\section{$4.360-70 \mathrm{~km}$}

Above the tropopause, the three dimensional structure of the temperature field becomes more complex. The most striking feature is represented by the 'cold collar' as a local temperature minima $(\sim 220 \mathrm{~K})$ centered at approximately $65 \mathrm{~km}$ altitude and $65^{\circ}$ latitudes on both hemispheres (Figs. 2, 3, 4, 5, 6, 15). The occurrence of cold collar is due to the presence of an inversion layer. Notably, the regions poleward of $80^{\circ}$ tend to often be isothermal between 60 and $70 \mathrm{~km}$ altitude at values around $235 \mathrm{~K}$ (Figs. 5 and 13). The altitude range of $60-70 \mathrm{~km}$ shows a constant negative temperature gradient only around the equator in the indicative latitude range $50^{\circ} \mathrm{S}-50^{\circ} \mathrm{N}$. The cold collar regions also have a marked local time structure, at least in the southern hemisphere: Figs. 3a, 4 and 6 consistently show that cold collar temperatures at 3:00 LT are about 8-1 0K colder than at 18:00 LT. This was also found by the earlier results from the Venera 15 FTS experiment (Schäfer et al. 1990), who reported a deeper inversion in the morning terminator than in the evening. Rise of temperature toward the terminators seen in the VIRTIS data are better interpreted in view of the findings reported in Tellmann et al. 2009. Two warm regions (about $240 \mathrm{~K}$ ) are clearly detected over the two terminators and centered at about $50 \mathrm{mb}$. Minimum air temperatures related to the cold collar are quantitatively consistent with VIRTIS estimates (i.e., the $210 \mathrm{~K}$ minimum at 0:00 LT and $90 \mathrm{mb}$ ). The sparser VeRa sampling is probably the cause of the missing detection of minima shift toward the dusk side (minimum is located at 0:00 LT instead of 3:00 LT).

\section{$4.470-90 \mathrm{~km}$}

Moving upward, the structure of the atmosphere becomes simpler: the vertical temperature gradient is constantly negative at all latitudes on the night hemisphere between 73 and $90 \mathrm{~km}$ (Figs. 2, 5 and 13). The atmosphere over the two poles is always warmer than at the equator and is due to the adiabatic heating from the subsidence caused by the convergence of the mean meridional flow of the poleward branch of the Hadley circulation. At the indicative level of $80 \mathrm{~km}$, the equatorial air temperature is around $205 \mathrm{~K}$ and $215 \mathrm{~K}$ at the poles (Fig. 5). While the large sample average results of VIRTIS data suggest that increase in temperature toward the poles is monotonous, the VeRa and SOIR data - benefitting from a better vertical resolution - seem to indicate that some latitudinal structure may exist at higher levels above $80 \mathrm{~km}$. The inverted temperature gradient in the mesosphere above the tropopause indicates 
decaying wind velocities with increasing altitude in the mid latitudes. The polar region displays a completely different but globally important regime with the dynamical instability features that manifest themselves periodically (Limaye et al. 2009).

In the altitude range between 70 and $90 \mathrm{~km}$, there is clear evidence of local time trends (Figs. 3, 7 and 22). At $70 \mathrm{~km}$ altitude and at a fixed latitude between $55^{\circ} \mathrm{S}$ and $70^{\circ} \mathrm{S}$, we observe minimum temperatures between 2:00 LT and 3:00 LT, with temperatures increasing toward both terminators, with differences in the order of 5-8 K. In a wider view, at $70 \mathrm{~km}$ altitude, the dusk hemisphere appears warmer for all latitudes poleward of $70^{\circ} \mathrm{S}$. At $75 \mathrm{~km}$, the local time structure appears to become more uniform, while at $80 \mathrm{~km}$ the dawn hemisphere becomes the warmer one, and is about $10 \mathrm{~K}$ warmer at $60^{\circ} \mathrm{S}$. In the equatorial region, a pronounced semidiurnal structure is found (Schofield and Taylor 1983; also Fig. 21) with a temperature minima of $\sim 165-170 \mathrm{~K}$ located near the morning and evening terminator at $\sim 90-95 \mathrm{~km}$.

\subsection{Above $90 \mathrm{~km}$}

Large temporal variability and local time dependence are the two main characteristics of the thermal structure above $90 \mathrm{~km}$ and extending to about $120 \mathrm{~km}$. Temperature increases initially until about $103 \mathrm{~km}$ and then decreases until $\sim 110 \mathrm{~km}$ altitude. Although ozone has been detected on Venus (Montmessin et al. 2011) around $100 \mathrm{~km}$, its abundance is too low to account for the inversion. The presence of aerosols and subsidence resulting from global circulation are the more likely contributors. The inversion layer seen around the $94 \mathrm{~km}$ level in SOIR results is also seen in ground based observations of thermal structure (Clancy et al. 2012, 2015) obtained using sub-millimeter wavelength observations of molecular rotational lines.

The Venus thermosphere, which extends from $120 \mathrm{~km}$ to $200 \mathrm{~km}$, is mainly composed of $\mathrm{H}, \mathrm{He}, \mathrm{O}, \mathrm{CO}_{2}, \mathrm{CO}, \mathrm{N}$ and $\mathrm{N}_{2}$. It is characterized by a hot dayside and a very cold nightside, separated by a sharp and key transition region, the terminator. Kasprzak et al. (1997) have previously discussed the neutral composition, thermal structure, dynamics and effects of the solar activity and Marcq et al. (2018) present an update on the composition and chemistry of the neutral atmosphere. Since then, the SOIR infrared (Mahieux et al. 2008; Nevejans et al. 2006) and SPICAV-UV (Bertaux et al. 2007a, 2007b) spectrometers, the aerobraking experiment VEXADE (Müller-Wodarg et al. 2006) and the torque experiment (Persson 2015; Rosenblatt et al. 2012) of the Venus Express mission focused on studying the composition, mass density and temperature structure of the thermosphere. Ground based campaigns also collected observations during the spacecraft observation periods such as the ones from the THIS heterodyne spectrometer (Sonnabend et al. 2012). Aside from measurements, the 3D Venus Thermosphere Global Circulation Model (VTGCM, Bougher et al. 1988) reproduces the main characteristics of the Venus thermosphere circulation, thermal structure and composition.

$\mathrm{CO}_{2}$ is still the dominant species of the Venus thermosphere up to an altitude of $\sim 140 \mathrm{~km}$ on the nightside and $\sim 155 \mathrm{~km}$ on the dayside (Niemann et al. 1980; Kasprzak et al. 1997) but in decreasing amounts at higher altitudes (Niemann et al. 1980; Seiff and Kirk 1982). The composition has been observed to vary with local time from the Pioneer Venus Orbiter and Bus measurements, but the sampling in local time is sparse above $140 \mathrm{~km}$. Above these altitudes, atomic oxygen becomes the main species, causing a significant change in the local molecular weight (Fig. 40). It is produced on the dayside by solar UV photo dissociation of $\mathrm{CO}_{2}$, and is transported to the night side by the sub-solar to anti-solar circulation (Bougher et al. 1986), which is found to be the major circulation pattern in this region. 
No instruments on-board Venus Express were designed to study the dayside thermosphere. The SOIR instrument, which sounded the Venus atmosphere using solar occultations, retrieved composition and temperature profiles on both sides of the Venus terminator, covering all latitudes, in the altitude region from 70 to $170 \mathrm{~km}$. The instrument measured infrared transmittances, from which $\mathrm{CO}_{2}$ number density profiles are inferred, as well as vertical distribution of other species (Mahieux et al. 2015a; Mahieux et al. 2012; Vandaele et al. 2015). The temperature profiles are calculated assuming the hydrostatic equilibrium, which is thus a secondary product. The SPICAV-UV used stellar occultations to study the nightside thermosphere, covering all latitudes in the altitude, from 90 to $140 \mathrm{~km}$ (Piccialli et al. 2014). UV transmittances are measured, from which $\mathrm{CO}_{2}$ number density profiles are inverted. As for SOIR, the hydrostatic equilibrium and constant mean molecular weight are assumed to calculate the number density profiles. For both instruments, the retrieval technique needs to assume the mixing ratio of $\mathrm{CO}_{2}$, which is taken from Keating et al. (1980). The VEXADE and torque experiments measured the total mass density of the atmosphere in the North polar region, around the apogee of the Venus Express orbit, during the aerobraking phases (Persson 2015; Rosenblatt et al. 2012). The temperature was calculated assuming hydrostatic equilibrium (which requires a knowledge of the local mean molecular weight). The VEXADE and torque experiments probed the thermosphere in the North polar region in the $130-140 \mathrm{~km}$ region and in the $160-200 \mathrm{~km}$ region, respectively. The temperature profiles derived from the torque measurements are in relatively good agreement with the SOIR and SPICAV/UV measurements but show a strong dependence on the solar zenith angle and noticeable temporal variability. Results on temperatures retrieved from the Tunable Heterodyne Infrared Spectrometer (THIS) measurements have been compared with the SOIR temperatures by Sonnabend et al. (2012) and show good agreement within the respective uncertainties of both instruments.

The mean nightside thermosphere temperature profiles show a negative gradient between 90 and $130 \mathrm{~km}$, with a weak latitude or local solar time dependence (see Figs. 5 and 6 of Piccialli et al. 2014). Small scale structures are observed in the mean thermal field, but are probably sampling effects due to the lack of profiles in some latitude regions. The temperature field shows a mean trend when considered as a function of the altitude, with a decrease from the morning terminator to the evening terminator, with a maximum at 04:00 AM. The mean thermal structure on both sides of the terminator is characterized by a succession of warm and cold layers, which indicates this region is a transition zone between the warm dayside and the cold nightside. A very cold layer located at $\sim 125 \mathrm{~km}$ is always found, with temperatures decreasing to as low as 100 to $120 \mathrm{~K}$ (Mahieux et al. 2012, 2015a). These temperatures are colder than on the nightside at the same altitude level. A warm layer is also found in all observations at lower altitudes $(\sim 105 \mathrm{~km}, 220 \mathrm{~K})$, which is warmer than what is found at the same altitude level on the nightside. At lower altitudes, a cold layer is found at an altitude of $80 \mathrm{~km}$, with temperatures around $180 \mathrm{~K}$. No differences are observed in the mean temperature profiles between the morning and evening sides of the terminator. A weak latitudinal dependence of the lower warm layer $(\sim 105 \mathrm{~km})$ is observed on both sides of the terminator, with a maximum around $45^{\circ}$. No clear latitudinal dependence is seen at other altitude levels. The mean terminator temperature profiles have been compared with the 3D VTGCM model fields in the terminator region, with relatively good agreement (Bougher et al. 2015). The succession of warm and cold layers is well reproduced, but the altitude and the temperature of the minima and maxima are slightly different. An attempt to model the terminator thermal structure using a 1D radiative transfer model is presented in Mahieux et al. (2016), with relatively good results. The mean thermal structure can be reproduced if modes 1 and 2 aerosols are considered in the mesosphere and lower thermosphere (below 


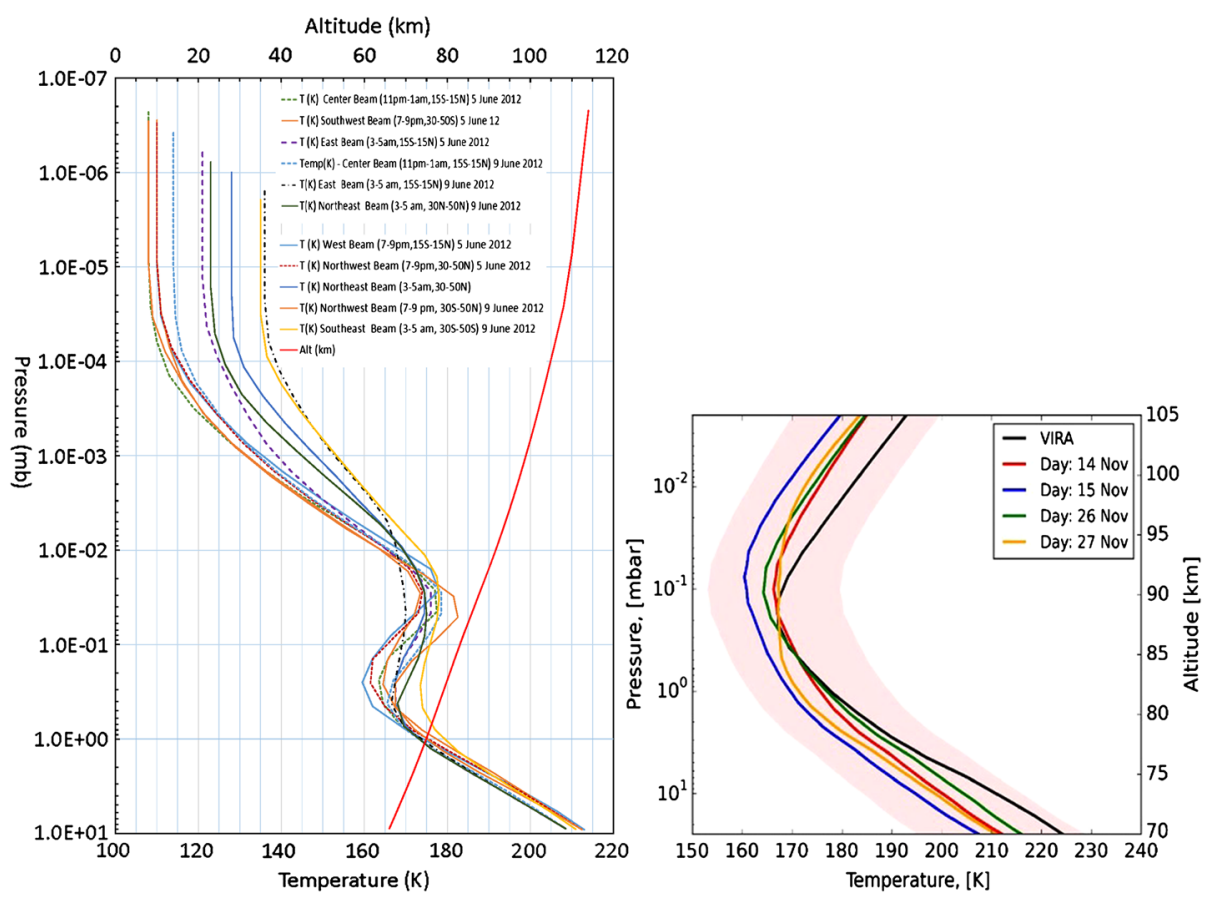

Fig. 26 (Left) Example of ground based inferences of atmospheric temperature using the JCMT (Clancy et al. 2015) on 5 and 9 June 2012 at different locations (and local times) on Venus. The corresponding altitude scale is shown as a black dashed line. (Right) Figure from Piccialli et al. (2017) showing profiles obtained from the Atacama Large Millimeter Array on four different days

$130 \mathrm{~km}$ ), which introduces a cooling mechanism. The aerosols modal and vertical distribution is in agreement with what has been measured by Wilquet et al. (2012). Titov et al. (2018) present a review of the recent progress in our knowledge of the cloud structure.

Large variabilities are observed in all datasets, mostly on the short term scale. The results of the atmospheric torque data suggest existence of gravity waves (Persson 2015). Large variabilities are observed in the SOIR temperature profiles, with a standard deviation around $40 \mathrm{~K}$ (Mahieux et al. 2015a, 2015b). The same temperature variability order of magnitude is observed in the SPICAV/UV profiles (Piccialli et al. 2014). The origin of these large variations is surprising but as yet no study to consider the origins or possible explanations has been performed on these datasets.

Ground based investigations of the thermospheric temperature structure provide some useful results despite the lower spatial resolution. Clancy et al. $(2012,2015)$ and Piccialli et al. (2017) have used the sub-millimeter CO lines to infer temperatures above $10 \mathrm{mb}$ level using different telescopes. Figure 26 (left) shows profiles of night time temperature obtained by Clancy et al. (2015) in different regions (and local times) of Venus on two different dates ( 5 and 9 June 2012) in between $\sim 65-115 \mathrm{~km}$ altitude. These compare well with the profiles obtained from the Atacama millimeter array (Piccialli et al., 2016) shown in the bottom panel.

Temporal Variability (Short-Term) of the Atmospheric Temperature fields Available datasets allow one to sample the air temperature fields with a typical minimum temporal 


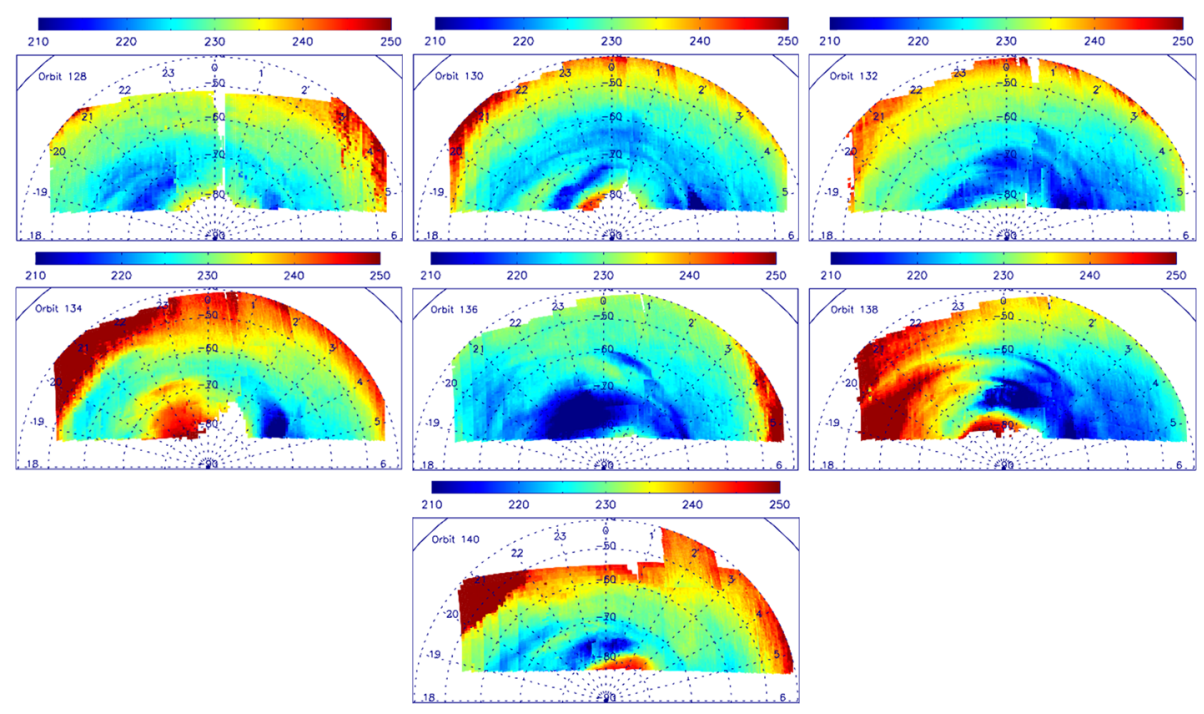

Fig. 27 Example of day to day variability at $98.6 \mathrm{mb}$ as seen from VIRTIS-M observations (Grassi et al. 2014, personal communication). The panels show thermal maps obtained on Venus Express orbits 128, 130, 132, 134, 136, 138 and 140 (each orbit is one day). The thermal patterns rotate in the planet's reference frame due to atmospheric circulation leading to local variations

step of an Earth day. In this frequency regime, both VeRa and VIRTIS data show regions of higher variability located at the indicative level of $65 \mathrm{~km}$ and immediately poleward of the cold collars (see Figs. 8 and 18, as well as Fig. 5 of Grassi et al. 2010). An example of the variability at $98.6 \mathrm{mb}$ level inferred from VIRTIS observations (Grassi et al. 2014) is shown in Fig. 27. Different absolute values for temperature fluctuations in the two datasets are related to differences in adopted definitions, instrument vertical resolution and filtering criteria adopted in defining the populations. At this altitude, fluctuations appear to show local time dependence: Grassi et al. (2010) indicate the dawn side as the most variable on the night hemisphere, while Tellmann et al. (2012) also report some local time dependence. Tellmann et al. (2012) ascribe this variability to the propagation of vertical waves, but data from VIRTIS-M (Fig. 27) also suggest that the passage of individual weather fronts may play an important role. As mentioned above in the VeRa results section, higher wave amplitude on the northern hemisphere is interpreted as an orographic effect triggered by the prominent Ishtar Terra. On the other hand, within the middle cloud, the low static stability impedes the propagation of atmospheric waves and only very shallow vertical temperature fluctuations are observed (Hinson and Jenkins 1995; Tellmann et al. 2012). Gravity waves seen above the middle cloud layer might therefore also be convectively generated in this middle cloud layer (Baker et al. 1998).

Thermal tidal structures play an important role in the equatorial region in the upper mesosphere, where prominent semidiurnal tidal temperature fluctuations are observed (Schofield and Taylor 1983). They likely play an important role in the maintenance of the atmospheric superrotation and the angular momentum budget. Local time variations in the thermal structure have been detected in the portions of the atmosphere from the cloud layer and above from occultation or infrared retrievals of thermal structure. Insufficient sampling from some investigation techniques may suggest temporal variations when in reality the variations may be due to thermal tides. In the lower atmosphere, from near the cloud base down to the sur- 
face there is not much spatial or temporal coverage. Given the long radiative time constant of the near surface layers, such variations are expected to be small, but further analysis of VIRTIS data are suggestive of some detectable changes (Mueller et al. 2018) in the near surface temperatures. Longer term variations (over a few solar cycles) in the atmospheric thermal structure cannot be ruled out due to solar cycle changes as well as possible changes in the albedo of the global cloud cover but there is insufficient data to establish a good average due to sampling deficiencies.

Grassi et al. $(2008,2010)$ pointed out another region of moderate temperature variability located at about $1 \mathrm{mb}, 65^{\circ} \mathrm{S}$ latitude. This finding was confirmed by Haus et al. (2014), while a similar region of higher fluctuation is seen in the VeRa data, but more evidently on the northern hemisphere. The SOIR data present a much more dynamical scenario for the upper mesosphere, with differences between consecutive observations up to a few tens of K (Fig. 13b) above $80 \mathrm{~K}$. The variability appears to diminish slightly toward the lower boundary of the probed altitude range and that terminator regions are likely subject to more marked variations.

We stress that significant opportunities for further data analysis exist on the available VIRTIS retrievals. Comparison of overlapping frames, albeit over limited areas, can provide insight into phenomena with time scales down to about twenty minutes. Long term trends can also be addressed based on data acquired during the 2.5 years operative lifetime of the instrument.

\section{Radiative Balance}

Observations, numerical modeling and theoretical studies have all revealed the extremely important role that radiation plays in various Venus processes, both now and early in its history. The large opacity of the atmosphere and the presence of great amounts of radiatively active gases and aerosols give Venus its unique place among the terrestrial planets. The greenhouse mechanism has clearly been very effective in forming the current and early climate on Venus, while the peculiar distribution of the radiative energy sinks and sources drive the remarkable super-rotation of the entire atmosphere. Chemical interactions between different gaseous and aerosol species and the importance of non-linear feedbacks make the Venus climate a very complex system.

Measurements of the radiation field within and outside of the Venus atmosphere during the past several decades have produced great progress in our understanding of conditions on Venus and of the atmospheric processes on the planet. Remote sensing observations from ground-based telescopes and orbiter instruments described the spectral dependence of the solar radiation reflected from the planet as well as the thermal radiation emitted into space. In situ measurements of scattered solar and thermal radiation from Venera and PioneerVenus descent probes provided constraints on the vertical distribution of radiation within the atmosphere. Recent multi-wavelength observations by Venus Express provided important new clues on variations of the radiative energy balance. This section gives a synthesis of the available observations.

Previously, Titov et al. (2007) have reviewed the observations and gaps in our knowledge of the solar and thermal radiation fluxes in the Venus atmosphere just prior to the beginning of the Venus Express coverage. The VIRTIS (Piccioni et al. 2007a) and VMC on Venus Express (Markiewicz et al. 2007a, 2007b) have provided reflected solar and near infrared emission from Venus, however the coverage is not global due to the elliptic polar orbit from 
which the measurements were made, and provide a better coverage of the Southern hemisphere. VIRTIS near infrared observations ended after two years of operation but continued to provide reflected solar observations $(0.25-1.0 \mu \mathrm{m})$. Another experiment on Venus Express, the Planetary Fourier Spectrometer (Formisano et al. 2006), did not return any data due to a hardware problem (Svedhehm et al. 2009) but would have provided observations in the thermal range where Venus loses its heat to space $(8-15 \mu \mathrm{m})$. There have been no entry probes to Venus after the VeGa 1 and 2 landers (June 1985).

The radiative balance of Venus has received some interest in recent years. Haus et al. (2016) investigated the radiative energy balance of Venus using improved estimates of the thermal structure of middle and lower atmosphere from VIRTIS results and followed up (Haus 2017) with a parameterization scheme for heating and cooling rates which are useful for running Venus climate models. Read et al. (2016) presented an overview of the energy budgets for different planets in a comparative sense including results from a global circulation model (GCM) for Venus. The collected papers presented at the first Comparative Climatology of Terrestrial Planets conference organized by the Venus Exploration Analysis Group (VEXAG) ${ }^{1}$ includes many presentations that discuss the climate of Venus in a comparative context (Binzel 2013). Bullock and Grinspoon (2013) discussed the climate of Venus in the context of atmospheric loss and past presence of water on the surface. Covey et al. (2013) discuss the role of greenhouse effect and climate feedback for the terrestrial planets. Schubert and Mitchell (2013) discuss the energy and entropy exchange on Venus and point out that significant entropy production from precipitation is not expected from the clouds on Venus unlike that on Earth. Turbulent and viscous dissipation in the thick atmosphere of Venus must produce entropy to balance the radiative loss by the atmosphere. Breaking of the upward propagating waves at the tropopause triggered by the bottom topography may also play a role (Izakov 2010). VeGa balloons experienced considerable updrafts and downdrafts near $54 \mathrm{~km}$ and similar chaotic motions may occur in the deeper atmosphere. Night time images taken from Akatsuki orbiter show cloud morphologies (Limaye et al. 2017) which may be indicative of such motions.

\subsection{Outgoing Radiation}

The spectrum of Venus when observed from space can be roughly split into three components: reflected solar radiation, infrared thermal emission from the cloud tops, and emission from the hot deep atmosphere and the surface leaking into space through the narrow spectral transparency "windows". The first component dominates in the UV, through the near-infrared range $(0.2-4 \mu \mathrm{m})$ over the sunlit hemisphere, while the second one prevails at longer infrared wavelengths $(4-50 \mu \mathrm{m})$. The third component is located in the spectral range from $0.9-2.5 \mu \mathrm{m}$, and is by several orders of magnitude weaker than the solar reflected light. Figure 28 shows typical spectra of all three components.

\subsubsection{Reflected Spectrum}

Kuiper (1969) presented the spectrum of Venus between 0.2-4.0 $\mu \mathrm{m}$. Barker et al. (1975) presented relative spectrophotometry between $306.7-596 \mathrm{~nm}$ and the bright and dark contrast values over this range. Observations of the reflected solar spectrum of Venus and the inference of its spherical albedo were summarized by Moroz (1983), and used for the Venus International Reference Atmosphere (Moroz et al. 1985). A major fraction of the incoming

\footnotetext{
${ }^{1}$ http://www.lpi.usra.edu/vexag/reports/VEXAG_PSS_090312.pdf
} 
Fig. 28 General view of the Venus spectrum at a resolving power, $\delta \lambda / \lambda$, of 200 as seen from space: the reflected solar light (dashed line), the thermal emission from the cloud tops and mesosphere (dotted line), and the night side emission escaping from the lower atmosphere (solid line). (From Titov et al. 2013)

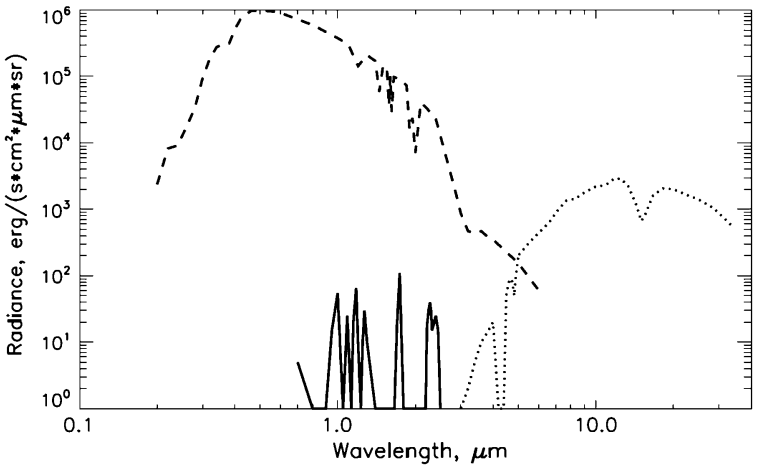

solar flux is scattered back to space by thick clouds that are mainly composed of sulfuric acid and completely cover the planet. Estimates of the Bond albedo vary from $0.80 \pm 0.02$ (Tomasko et al. 1980b) to $0.76 \pm 0.01$ (Moroz et al. 1985). Previously Irvine (1968) had obtained a value of $0.77 \pm 0.07$. The ground based estimates were based however on an incomplete phase angle coverage as low $\left(<30^{\circ}\right)$ and high phase angle $\left(>150^{\circ}\right)$ photometric observations of Venus are not possible from Earth based telescopes. Mallama et al. (2006) present some new results that for the first time extend the phase angle coverage to both low and high angles by including measurements from the SOHO spacecraft (as low as $2^{\circ}$ and as high as $179^{\circ}$ phase angle). They also argue that the previous measurements of the spectral albedo underestimated the brightness of Venus due to an observing effect caused by the size of the used aperture. However, the older and more recent measurements used different sensors and the difference can arise from a systematic bias. Mallama et al. observations however confirm the bump at high phase angles expected from the thick cloud cover of Venus which was inferred by Travis (1975) in his re-analysis of Irvine's (1968) measurements. In a subsequent paper Mallama (2009) reports the geometric albedo (the ratio of the brightness at zero phase angle to that of a Lambertian scatterer) for Venus to be 0.67 in the $\mathrm{V}$ band. Haus et al. (2016) estimate this value to be 0.764 from investigation of the radiative balance using improved cloud models on the middle and lower atmosphere of Venus, a parameterization of absorption due to the unknown absorbers and a model of the solar irradiance spectrum.

The spectral dependence of the Venus spherical albedo from historical observations is shown in Fig. 29. The albedo curve shows a broad depression in the UV-blue range. At wavelengths below $0.32 \mu \mathrm{m}$, this absorption is explained by the presence of $\mathrm{SO}_{2}$ (and $\mathrm{SO}$ ) gas within and above the cloud tops. The absorption at longer wavelengths $(0.32-0.5 \mu \mathrm{m})$ is attributed to the presence of unknown absorbers in the upper cloud $(58-65 \mathrm{~km})$. Variability of the upper cloud structure and abundance of the absorbing species produces the wellknown UV markings on the Venus disc with albedo variations of up to 30\% (Coffeen 1971; Woodman and Barker 1975; Limaye 1984; Rossow et al. 1980; Markiewicz et al. 2007a, 2007b; Titov et al. 2012). In the near infrared (1-2 $\mu \mathrm{m})$, sharp spectral features associated with absorption by $\mathrm{CO}_{2}$ and $\mathrm{H}_{2} \mathrm{O}$ within and above the cloud tops are clearly seen in the Venus spectrum. At wavelengths longer than $2.5 \mu \mathrm{m}$, the Venus albedo quickly drops to a few percent due to strong absorption by sulfuric acid aerosols (Fig. 28). Above $4 \mu \mathrm{m}$, thermal radiation emitted by the cloud tops becomes comparable to the reflected solar light and its intensity rapidly increases with wavelength.

Unfortunately, there have been few investigations in recent decades of the spectral albedo of Venus and although the value quoted by Moroz et al. (1985), there is some speculation regarding whether the albebo of Venus changes over time. This is a reasonable question as 


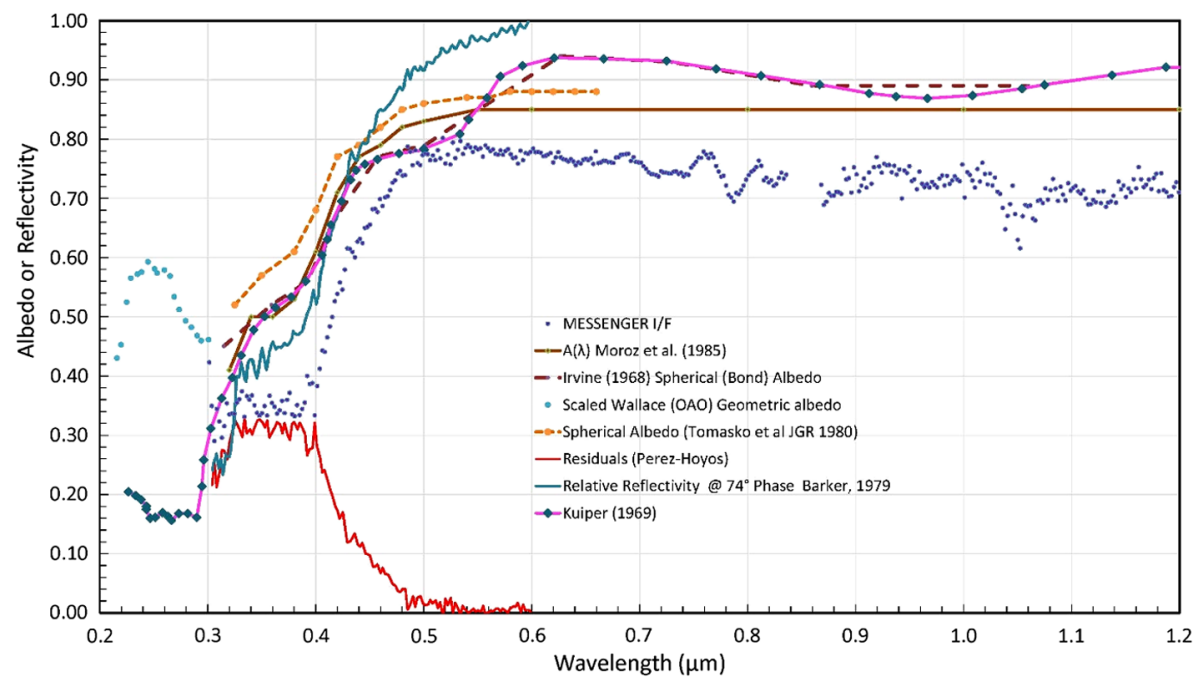

Fig. 29 Comparison of available measurements of spherical albedo or reflectance of Venus. Also shown in this figure is an estimate of the absorption of incident sunlight by difference of one MESSENGER reflectivity profile the expected spectrum from an assumed cloud model (from Pérez-Hoyos et al. 2017), also shows are the transmission spectrum of $\mathrm{S}_{\alpha}+\mathrm{SCl}_{2}$ (Krasnopolsky 1986) and Fe-S protein from A. Thiobacillus Ferrooxidans (Ossa et al. 2011) indicating that both inorganic and biological aerosols can match the observed short wave absorption in Venus clouds (Limaye et al. 2018b)

we now have observations of Venus from three orbiters - Pioneer Venus December 1978December 1983), Venus Express (June 2006-November 2014) and Akatsuki (April 2016present) which have imaged Venus at $365 \mathrm{~nm}$. Lee et al. (2015) have analyzed UV images from the VMC and concluded that the albedo at $365 \mathrm{~nm}$ decreased slightly over the coverage period, but this is not at odds with the results of the same data set presented by Shalygina et al. (2015) who looked into the degradation of the VMC degradation over its operating life. Bullock et al. (2007) explored the impact of changes in the planet's Bond albedo on the equilibrium surface temperature. However, it is recognized that short term changes in albedo (caused by changes in the cloud cover) should not impact the lower atmospheric or surface temperatures on short time scales due to the long radiative time constant, but the upper atmospheric temperatures can change on shorter time scales, and plausibly over a solar cycle during which the solar output at short wavelengths does show larger variations and thus any change in the Venus UV contrasts could lead to some changes.

\subsubsection{Absorbers of Incident Sunlight in Venus Clouds}

A key unknown here are the substances that absorb the incident solar radiation in the clouds of Venus. This was recognized early once the composition of the Venus atmosphere was inferred to be sulfuric acid (Hansen and Hovenier 1974) of varying concentrations (75$85 \%$ ) and that there must be additional absorbers present in the atmosphere (Travis 1975; Pollack et al. 1980a, 1980b) because sulfuric acid has no absorption in the ultraviolet-visible range. It is important to remember that this identification was based on the determination of the index of refraction of the cloud particles required to match the observed disk integrated polarization data obtained from earth based telescope by Lyot (1929) across wavelengths with the phase angle and matching retrieved the index of refraction with the values for 
candidate species. Observations of the glory feature in spacecraft observations of Venus have been used to estimate the variation of cloud particle properties over the disk (Markiewicz et al. 2014; Petrova et al. 2015). However, this approach is not very sensitive to determine the index of refraction according to Laven (2005).

The uncertainty in the identity of the absorber(s) responsible for the absorption in the ultraviolet has been discussed by Esposito (1980) and Esposito et al. (1983). Sulfur dioxide was detected in the atmosphere of Venus through its absorption below $330 \mathrm{~nm}$ by Barker (1979) and is one of the absorbers of incident sunlight. Carbon disulfide $\left(\mathrm{CS}_{2}\right)$ was detected earlier by Barker (1978) and confirmed by Hua et al. (1979a) by its absorption feature at $315 \mathrm{~nm}$. More than a dozen other candidates have been proposed as possible absorbers, including microorganisms (Limaye et al. 2018b), and sulfur dioxide which absorbs below $320 \mathrm{~nm}$ has been identified above the clouds and within the cloud. $\mathrm{ClO}$, which has been recently detected from sub-millimeter spectroscopy by Sandor and Clancy (2018), also absorbs at ultraviolet wavelengths and is found to exhibit much larger abundances in the upper (85-100 km) versus lower (70-85 km) mesosphere. Previously, Toon et al. (1982) proposed that elemental sulfur can be formed from sulfur dioxide above the clouds and could form aerosols, which could be the small particles detected by the Pioneer Large Probe (Knollenberg and Hunten 1980) and can be another absorber. Carlson (2016) suggested that polymeric and octasulfur $\left(\mathrm{S}_{8}\right)$ are consistent with the VIRTIS spectra and may be consistent with the presence of $\mathrm{FeCl}_{3}$ considered previously (Kuiper 1969; Zasova et al. 1981; Krasnopolsky 2017).

Abundance of sulfur dioxide above cloud tops has been observed to vary over short time scales ( $\sim 1$ hour) and spatially (Encrenaz et al. 2016) and over years (Marcq et al. 2013; Vandaele et al. 2017). Images obtained at $270 \mathrm{~nm}$ (Limaye 1984) from the Orbiter Cloud PhotoPolarimeter (OCPP) by Pioneer Venus and those taken by the UVI camera on Akatsuki orbiter (Yamazaki et al. 2018; Limaye et al. 2018b) show contrast features similar in morphology at times and different from those seen at $365 \mathrm{~nm}$ (where there is no absorption by sulfur dioxide), confirming that other absorbers must be present. Further, the differences are also likely in their vertical location, as the sulfur dioxide has been measured within and above the clouds (Encrenaz et al. 2016).

The two favored inorganic candidates include sulfur (most recently favored by Carlson 2016 and many others previously) and $\mathrm{FeCl}_{3}$ favored by Krasnopolsky (2017). However, Petrova (2018) notes that elemental sulfur is not wetted by sulfuric acid and cannot be responsible for contrasts as sulfur particles can only adhere to the sulfuric acid droplets, which would distort the glory feature. Grains of other absorbers can be embedded (as condensation nuclei) in the droplets to explain the inferred index of refraction and the observed glory feature.

Conceivably the nuclei can also be microorganisms as their physical characteristics can be similar to the cloud particles found in the Venus clouds. Many terrestrial microorganisms rely on Fe-S chemistry and some could have evolved independently on Venus when it had liquid water on the surface). Sulfur based aerosols are believed to have played a role in the evolution of early life on Earth (Farquhar et al. 2000; Lyons 2008) and Kumar and Francisco (2014) propose a non-photochemical process for elemental sulfur aerosols which may occur on Venus. The question of the absorbers of sunlight in the Venus cloud layer is still very much unsettled.

\subsubsection{Infrared Emission}

Even though Venus has a very high surface temperature, from space it appears as a rather cold object because the sulfuric acid clouds are opaque at $\lambda>2.5 \mu \mathrm{m}$ and the temperature of 
Fig. 30 Examples of the Venus thermal infrared spectra measured by the FTS on

Venera $15\left(250-2600 \mathrm{~cm}^{-1}\right)$ and NIMS/Galileo experiments in the equatorial (solid) and middle (dashes) latitudes. The NIMS spectra (1900-2500 $\left.\mathrm{cm}^{-1}\right)$ are multiplied by a factor of 100 . Thin solid lines show the blackbody spectra for the temperatures of $260 \mathrm{~K}, 240 \mathrm{~K}$, and $220 \mathrm{~K}$

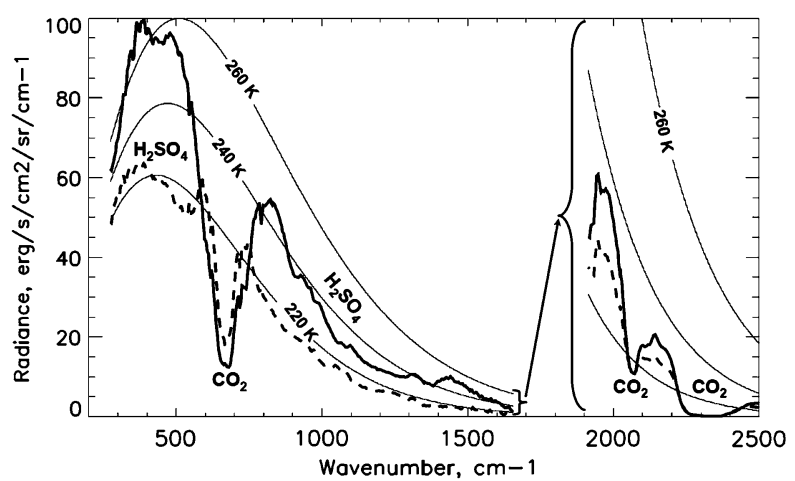

the cloud tops vary between $220 \mathrm{~K}$ and $260 \mathrm{~K}$. Venus thermal radiation has been measured both from the ground and from space. The most comprehensive data sets were delivered by the Pioneer Venus Orbiter Infrared Radiometer (Taylor et al. 1980), the Venera 15 Fourier spectrometer (Oertel et al. 1987), the NIMS/Galileo infrared spectro-imager (Carlson and Taylor 1993), and the VIRTIS on Venus Express (Drossart et al. 2007; Piccioni et al. 2007a). Figure 30 shows examples of the thermal infrared spectra of Venus. The spectrum of the planet at thermal wavelengths $(5-50 \mu \mathrm{m})$ is close to that of a blackbody at the cloud top, with temperatures and spectral features mainly belonging to mesospheric $\mathrm{CO}_{2}, \mathrm{H}_{2} \mathrm{O}, \mathrm{SO}_{2}$, as well as other gases that absorb at levels within and above the clouds as broad signatures of sulfuric acid aerosols. The fundamental absorption bands of $\mathrm{CO}_{2}$ at $4.3 \mu \mathrm{m}, 4.8 \mu \mathrm{m}$, and $15 \mu \mathrm{m}$ produce the strongest spectral features. These bands have been used to retrieve the temperature and aerosol altitude profiles in the mesosphere (Roos-Serote et al. 1995; Zasova et al. 2007b; Grassi et al. 2014). The thermal structure of the Venus mesosphere varies strongly with latitude, with polar regions surprisingly being up to $20 \mathrm{~K}$ warmer than equatorial latitudes at most pressure levels between the cloud tops and $100 \mathrm{~km}$. The thermal structure varies to a lesser extent with local time. Thermal infrared spectra also show a wealth of weaker absorption features belonging to hot and isotopic bands of $\mathrm{CO}_{2}$ as well as $\mathrm{SO}_{2}, \mathrm{H}_{2} \mathrm{O}$, and $\mathrm{CO}$ above the clouds and within the upper cloud. The thermal emission spectra in Figure clearly show the atmospheric parameters and constituents that play the major role in radiative energy exchange with space. Thermal radiation leaves the planet mainly in the spectral range from $200 \mathrm{~cm}^{-1}$ to $1000 \mathrm{~cm}^{-1}(50-10 \mu \mathrm{m})$. Strong infrared bands of $\mathrm{CO}_{2}$ clearly seen in the thermal emission spectra in Fig. 30 are formed in the colder mesosphere and are responsible for cooling of the atmosphere above the clouds (up to $\sim 120 \mathrm{~km}$ ).

An efficient atmospheric greenhouse mechanism maintains Venus surface temperatures as high as $750 \mathrm{~K}$. The accepted globally average surface temperature of Venus is $735 \mathrm{~K}$ (Kliore et al. 1985). However, the lowest plains can have surface temperatures of at least $750 \mathrm{~K}$. At these temperatures, the surface emits substantial amounts of energy even in the near infrared range $(1-5 \mu \mathrm{m})$. This emission was detected for the first time by the spectrophotometers onboard the Venera landers as an increase in the measured spectra at wavelengths above $0.8 \mu \mathrm{m}$. However, its importance was fully recognized only after Allen and Crawford (1984) discovered that thermal radiation from the lower atmosphere leaks to space through partially transparent atmospheric "windows" - spectral gaps between strong $\mathrm{CO}_{2}$ and $\mathrm{H}_{2} \mathrm{O}$ absorption bands in the near infrared range. The discovery of emissions on the Venus night side provided a powerful remote sensing tool to study composition of the lower atmosphere, to map the surface, and to monitor the cloud opacity and atmospheric dynamics 


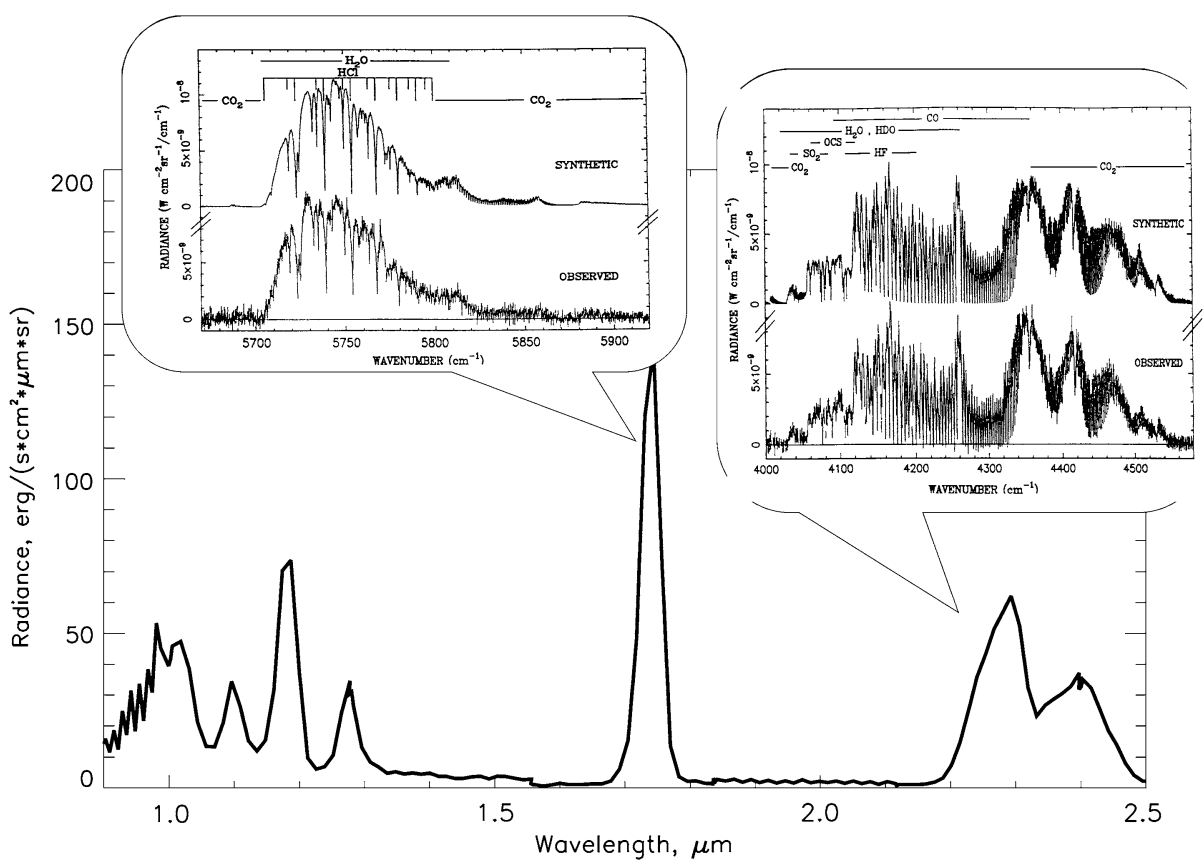

Fig. 31 NIMS/Galileo near infrared spectrum of the Venus night side. Ground based high resolution spectra of the emissions at $1.7 \mu \mathrm{m}$ and $2.3 \mu \mathrm{m}$ (from Bezard et al. 1990) are shown in the insets

in the cloud depth. This method has been used successfully by Venus Express (Titov et al. 2009).

At wavelengths of $0.8-2.4 \mu \mathrm{m}$, the $\mathrm{H}_{2} \mathrm{SO}_{4}$ clouds are translucent. Aerosols are almost non-absorbing and their scattering properties have little variability with wavelength. The altitude of the origin of the night side emission is wavelength dependent and varies from the very surface at $1 \mu \mathrm{m}$ to $\sim 35 \mathrm{~km}$ at $2.3 \mu \mathrm{m}$. These emissions are about 4 orders of magnitude weaker than the reflected solar component (Fig. 31) so they can be observed only on the night side. Figure 31 shows examples of the Venus night side spectra measured by NIMS/Galileo (Carlson and Taylor 1993) and high resolution spectra of the same spectral "windows" from the ground-based observations (Crisp 1989; Crisp et al. 1991a, 1991b; Bezard et al. 1990; Meadows and Crisp 1996; Taylor et al. 1997).

Five years of continuous monitoring of the outgoing radiation field by Venus Express revealed strong changes of the atmospheric parameters (albedo, temperature, cloud top structure) that results in significant variations of the global radiative energy budget of the planet. The Venus Monitoring Camera (VMC) imaged the planet in the narrow band filter centered at the characteristic band of the unknown UV absorber $(365 \mathrm{~nm}$ ) (Markiewicz et al. 2007a, 2007b; Titov et al. 2012) which is responsible for deposition of about half of the solar energy that Venus receives from the Sun. Inhomogeneous distribution of the absorber at the cloud tops produces famous UV markings and strongly affects the radiative energy deposition pattern (see Titov et al., this issue). The observed latitudinal variations of the UV albedo imply abrupt decrease in deposited solar energy by a factor of 3-4 from dark tropics to bright high latitudes on top of a gradual decrease due to solar incidence angle. The energy is deposited at the cloud tops and is not buffered by the bulk of the atmosphere. This can trigger a local dynamical response causing wind across the sharp albedo boundary - 
Fig. 32 Composite false color view of the Southern hemisphere: VIRTIS thermal-IR $(5 \mu \mathrm{m})$ mosaic (red) and simultaneously captured VMC UV $(0.365 \mu \mathrm{m})$ image (grey). The peak emission is seen in the core region of the hemispheric vortex roughly centered over the pole corresponding to lower cloud tops and descending air (and concurrent adiabatic warming) as the mean meridional flow converges

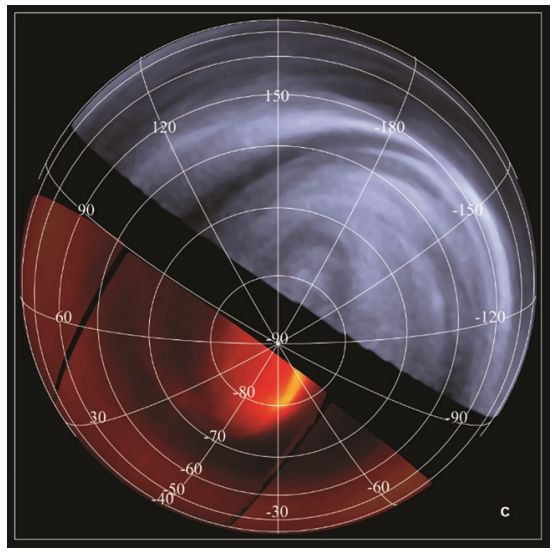

a mechanism similar to the mesoscale winds at the sea coasts on Earth or at the edge of the Martian polar cap. Such rare global brightening events, when bright haze almost completely covered Venus in about a day (Markiewicz et al. 2007a, 2007b) cause global changes of the planetary albedo that affects the radiative energy deposition pattern and thus has an impact on the atmospheric dynamics.

Figure 32 shows the outgoing thermal flux in the near infrared on the night side and the clouds/aerosol distribution on the day side. The emission is affected by the temperature and aerosol distribution at the cloud top level. Venus Express observations revealed that both parameters change significantly with latitude, resulting in strong modulation of the thermal emission. Radio-occultation experiment showed that the temperature structure changes from a monotonic profile in low latitudes to one with strong temperature inversions (Tellmann et al. 2009) that creates an annulus of cold air at 60-70 degrees latitude ("cold collar"). The cloud top structure changes in a correlated way with the temperature field. In low latitudes, the cloud top is located at $\sim 72 \mathrm{~km}$ and the aerosol vertical scale height is about $4 \mathrm{~km}$. In the "cold collar" the cloud top descends to $\sim 64 \mathrm{~km}$ and has a very sharp boundary coinciding with the altitude of the temperature minimum (Ignatiev et al. 2009; Lee 2011). This combination of changes in the temperature and cloud structures results in the modulation of thermal flux observed from orbit. Figure 32 shows the VIRTIS thermal infrared $(5 \mu \mathrm{m})$ mosaic captured by VIRTIS/Venus Express (Piccioni et al. 2007b). The brightness temperature in low latitudes is a few tenths of degrees higher than the cold collar region at $60-70^{\circ} \mathrm{S}$. The polar eye of the planetary vortex is the brightest at thermal IR wavelengths. Comparison to the earlier observations indicate that this distribution of thermal flux is roughly similar to that in the Northern hemisphere (Taylor et al. 1980).

Leaking of the thermal radiation into space from the hot lower atmosphere and the surface makes a minor contribution to the global radiative energy balance due to the weakness of the emission escaping through the thick cloud layer (Fig. 32). However, the radiative effect of the lower atmosphere thermal emission on the lower cloud could be much stronger. Measurements of the flux in the near-IR transparency "windows" from orbit give an important clue on distribution of opacity over the globe. Figure 33 shows the maps of the emissions in the $2.3 \mu \mathrm{m}$ "window". The VIRTIS/Venus Express mosaic of the southern hemisphere (left) indicates a factor of a 5-7 decrease of the flux from low and middle latitudes to the polar regions that corresponds to about a factor of 2 increase in the cloud opacity. The NIMS/Galileo image (right) shows the view of Venus from the equator indicating patchy morphology of the deep cloud. 

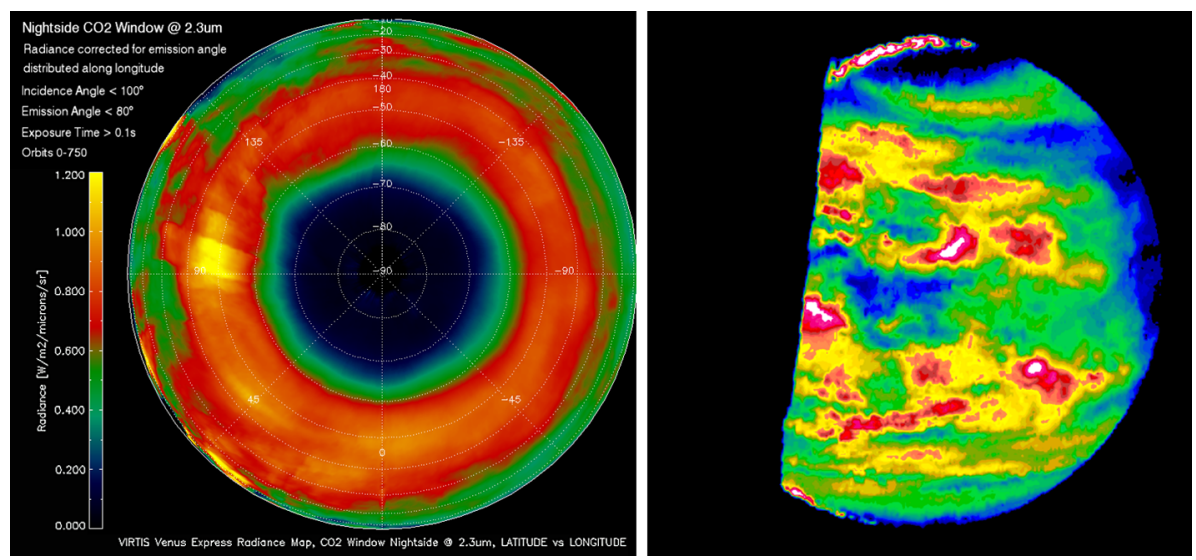

Fig. 33 Maps of the thermal emission escaping to space in the $2.3 \mu \mathrm{m}$ "window" captured by VIRTIS/Venus Express (left, from Cardesín Moinelo et al. 2008) and NIMS on Galileo orbiter (right, from Carlson et al. 1993). False colors mark variations of brightness produced by spatial inhomogeneity of the total cloud opacity. Red - high flux and low cloud opacity, blue - low flux and high cloud opacity

\subsection{Radiation Field Inside the Atmosphere}

The solar radiation field inside the atmosphere was measured by spectrophotometers on several Venera descent probes (Ekonomov et al. 1984; Moroz et al. 1983). These observations provided a unique data set for characterizing the angular and spectral distribution of solar scattered light between 0.4 and $1.2 \mu \mathrm{m}$ from $\sim 65 \mathrm{~km}$ down to the surface. The intensity of solar radiation propagating downwards gradually decreases with altitude. Less than $10 \%$ of the radiation that hits the top of the atmosphere reaches the surface and only about $2.5 \%$ $\left(\sim 17 \mathrm{~W} \mathrm{~m}^{-2}\right)$ is absorbed at the ground (Crisp and Titov 1997). Increasing absorption in near-infrared by $\mathrm{CO}_{2}$ and $\mathrm{H}_{2} \mathrm{O}$ as well as absorption at the blue end of the spectrum is also evident as the probe descends. Analysis of these data supported the conclusion from ground-based near-infrared observations (de Bergh et al. 1995) that the $\mathrm{H}_{2} \mathrm{O}$ mixing ratio is nearly constant $(30 \pm 10 \mathrm{ppm})$ at altitudes between the cloud base and $\sim 16 \mathrm{~km}$ but shows a probable increase up to 50-70 ppm in the lower scale height (Ignatiev et al. 1997).

Vertical profiles of the solar downward and upward fluxes and their divergence provide valuable information about the structure of the atmosphere and solar heating rates. The solar flux radiometer (LSFR) aboard the Pioneer Venus Large Probe measured solar fluxes during descent in a series of channels covering a broad range from 0.4 through $1.8 \mu \mathrm{m}$ (Tomasko et al. 1980a). The Venera-13 and 14 descent probes also carried a photometric experiment to measure the distribution of UV radiation (Ekonomov et al. 1984). The measurements of the vertical profiles of downward solar fluxes by the Venera and Pioneer-Venus descent probes showed that UV and blue radiation are strongly absorbed in the upper cloud layer above $57 \mathrm{~km}$. To find the global mean net solar flux, numerical models must be used to extend the spectral and spatial coverage provided by the experimental results (Tomasko et al. 1980a; Moroz et al. 1985). The measurements and subsequent radiative transfer modeling revealed two regions where deposition of the solar energy is significant. The first one is located in the upper cloud layer above $\sim 57 \mathrm{~km}$ and is possibly caused by the unknown UV absorber and by absorption beyond $3 \mu \mathrm{m}$ by the $\mathrm{H}_{2} \mathrm{SO}_{4}$ cloud particles. The second region is located in the lower atmosphere (40-20 km) where solar energy is absorbed by $\mathrm{CO}_{2}$ and $\mathrm{H}_{2} \mathrm{O}$ in the near-infrared and possibly elemental sulfur in the UV-blue range. Surprisingly, almost 
no absorption of sunlight occurs between 57 and $48 \mathrm{~km}$ where the bulk of the cloud layer is located.

The vertical distribution of thermal fluxes depends on the profiles of temperature and opacity sources in the atmosphere. The vertical net thermal flux divergence defines the thermal cooling rate, which, in combination with net solar heating, determines the radiative energy balance in the atmosphere. Experimental characterization of the thermal flux was the goal of three net flux radiometers (SNFR) on the Pioneer Venus Small Probes (Suomi et al. 1980) and an infrared radiometer (LIR) on the PV Large Probe (Boese et al. 1979). The original data were affected by a significant error whose source was discovered in laboratory testing after the mission was completed. Revercomb et al. (1985) corrected the measurements and analyzed their implications for the cloud structure, water vapor distribution in the lower atmosphere, and radiative cooling.

The measured thermal fluxes indicate the presence of an additional (either gaseous or particulate) source of opacity above $60 \mathrm{~km}$. Below the clouds, measurements suggest a strong increase of the net flux and its divergence from Equator to Pole. Because the temperature structure below the clouds varies little with latitude, this trend implies a strong latitude variability of infrared opacity sources. Since sulfur dioxide has fairly low absorption, Revercomb et al. (1985) concluded that the net flux measurements imply a significant latitude trend in the water mixing ratio below the clouds. This conclusion however, contradicts the results of a recent re-analysis of spectrophotometry on the Venera descent probes (Ignatiev et al. 1997) and observations in the near IR windows (Crisp et al. 1991b; Drossart et al. 1993; de Bergh et al. 2006; Bezard et al. 2009, 2011) which imply an $\mathrm{H}_{2} \mathrm{O}$ mixing ratio of $\sim 30 \mathrm{ppm}$ without significant latitude variability below the clouds. Bell et al. (1991) report no detection of variability water vapor in the deep atmosphere, but other the near IR observations show that there is significant spatial variability of the cloud opacity, with persistently low optical depths at latitudes between 40 and $60^{\circ}$ (Crisp et al. 1991b). The low cloud optical depths at latitudes where the Pioneer Venus North Probe entered the atmosphere may explain the large thermal net flux divergences near the cloud base inferred from the net flux radiometer results (Crisp and Titov 1997).

\subsection{Global Radiative Energy Balance}

Observations by the Pioneer Venus and Venera orbiters and descent probes provided a substantial amount of information about scattering and absorption properties of the Venus atmosphere. Several comprehensive radiative transfer models consistent with the data were developed at that time (Tomasko et al. 1980a, 1980b, 1985). The authors calculated the global balance of radiative energy and solar heating rates in the atmosphere. The total solar flux at the Venus orbit is $2622 \pm 6 \mathrm{~W} \mathrm{~m}^{-2}$ (Moroz et al. 1985). Due to its high albedo the planet absorbs only $157.66 \mathrm{~W} \mathrm{~m}^{-2}$ on average, less than that deposited on Earth $\left(\sim 240 \mathrm{~W} \mathrm{~m}^{-2}\right)$, despite the fact that Venus is $30 \%$ closer to the Sun. Both models and observations show that less than $10 \%$ of the total solar energy incident on Venus reaches the surface. The largest portion of solar energy is absorbed above $57 \mathrm{~km}$ by the unknown UV absorber at the cloud tops. This is in contrast with the Earth, where 74\% of the solar energy is absorbed directly at the ground level (Arking 1996).

The outgoing thermal radiation has been characterized by the Pioneer Venus Orbiter Infrared Radiometer (Taylor et al. 1980), the Venera 15 Fourier transform spectrometer (Oertel et al. 1987), and VIRTIS/ Venus Express (Drossart et al. 2007). These observations were analyzed to retrieve the temperature and aerosol structure of the Venus mesosphere and to calculate the outgoing thermal flux (Schofield and Taylor 1982; Zasova et al. 2007a; 
Fig. 34 Incident and emitted energy for Venus and Earth. (From Taylor et al. 1983.) Values for Earth have been updated recently by Wild et al. (2013)

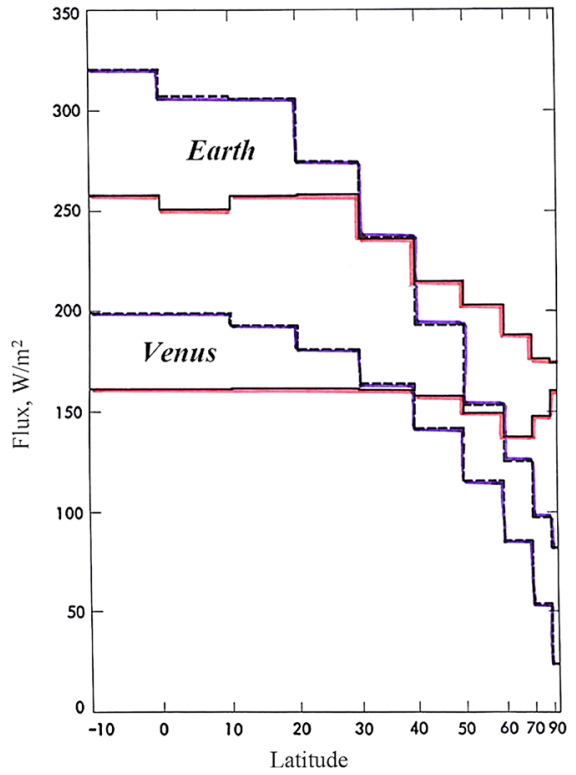

Grassi et al. 2014; Tellmann et al. 2009). The globally averaged effective temperature as measured by Pioneer Venus is $\sim 230 \mathrm{~K}$, which corresponds to an outgoing thermal flux of $\sim 160 \mathrm{~W} \mathrm{~m}^{-2}$. Haus et al. (2016) estimate the flux to be $156.7 \mathrm{~W} \mathrm{~m}^{-2}$. This value is slightly different from the mean solar flux deposited on the planet, but given the uncertainties in both values, this discrepancy cannot be interpreted as an indication of global energy imbalance. Venus Express delivered a significant amount of data on the outgoing thermal emission, especially in the Southern polar regions which showed the highly variable thermal structure of the polar eye of the global vortex (Garate-Lopez et al. 2015).

Available observations and models quantify the planetary radiation budget (Tomasko et al. 1980b; Schofield and Taylor 1982). Figure 34 compares the mean latitude distributions of solar radiation received by the planet and thermal radiation emitted to space for Venus and Earth. Wild et al. (2013) discuss Earth's mean energy balance using both surface stations and spacecraft observations and infer new estimates of global mean surface radiation. Data from the LIR camera on Akatsuki orbiter (Fukuhara et al. 2011) measure the emitted radiation by Venus between $8-12 \mu \mathrm{m}$. These data are still being gathered and soon analysis of these data should result in newer estimates of the emitted radiation emitted by Venus as a function of latitude. Both planets receive solar energy mainly at low latitudes with the incident flux strongly decreasing toward the poles. Earth has a similar trend in latitude distribution of the outgoing thermal radiation. On Venus however, the outgoing thermal flux is almost constant with latitude, indicating that atmospheric dynamics are more efficient in transporting energy from Equator to Pole.

The vertical divergence of the net solar and thermal fluxes gives the radiative heating and cooling rates in the atmosphere. These sources and sinks of radiative energy force the atmospheric dynamics. Radiative transfer models of the Venus atmosphere have progressed together with the accumulation of observational data (Pollack et al. 1980a, 1980b; Tomasko 1983). Tomasko et al. (1985) used an atmospheric radiative transfer model derived from the Pioneer-Venus and Venera measurements to calculate solar fluxes and heating rates (Fig. 35).

Observations by the Pioneer Venus, Venera 15 and Venus Express orbiters significantly improved our knowledge of the temperature and aerosol structure of the mesosphere 
Fig. 35 Vertical profiles of globally averaged solar heating (solid line - Tomasko et al. 1985; dashed line - Crisp and Titov 1997) and thermal cooling rates (dotted line - Crisp and Titov 1997). Haus et al. (2014) also provide similar estimates

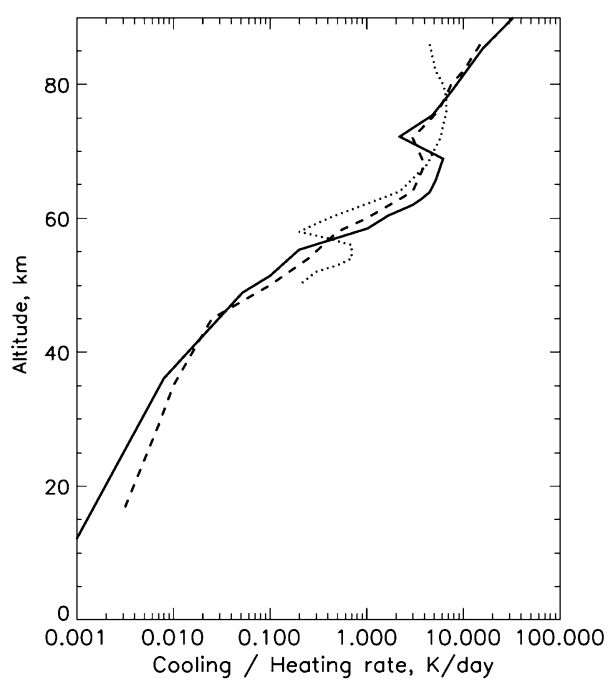

(Schofield and Taylor 1982; Zasova et al. 2007b; Grassi et al. 2008; Ignatiev et al. 2009; Lee et al. 2012; Tellmann et al. 2009). This inspired extensive numerical modeling of the radiative balance in the Venus mesosphere (Crisp 1986, 1989; Haus and Goering 1990; Titov 1995; Crisp and Titov 1997; Lee 2011). These studies confirmed that the radiative forcing is very sensitive to atmospheric parameters such as temperature structure, aerosol composition and distribution, and abundance of trace gases.

Figure 35 shows the vertical profiles of globally averaged cooling and heating rates in the Venus atmosphere. Above the clouds, the radiative energy exchange occurs mainly in the $\mathrm{CO}_{2}$ bands. Both cooling and heating rates gradually decrease from $\sim 50 \mathrm{~K} \mathrm{day}^{-1}$ near the top of the mesosphere $(90 \mathrm{~km})$ to a few $\mathrm{K} \mathrm{day}^{-1}$ at the cloud tops $(70 \mathrm{~km})$. Absorption by the unknown UV absorber and the $\mathrm{H}_{2} \mathrm{SO}_{4}$ cloud particles above $57 \mathrm{~km}$ in the upper cloud creates a heating rate peak of up to $8 \mathrm{~K} \mathrm{day}^{-1}$ in the global average. About $10 \%$ of the solar energy incident on the top of the atmosphere or half of the solar energy absorbed by the planet is deposited in this altitude range. Radiative cooling cannot compensate for this heating at low latitudes, creating a region of net heating in the upper cloud. Below the clouds $(<48 \mathrm{~km})$, both cooling and heating rates gradually decrease from $0.1-1 \mathrm{~K} \mathrm{day}^{-1}$ at the cloud base to $\sim 0.001 \mathrm{~K} \mathrm{day}^{-1}$ at the surface. Figure 36 shows the altitude-latitude field of net radiative heating calculated for the mesospheric temperature structure retrieved from the Pioneer Venus OIR remote sensing data (Crisp 1989). The observed thermal structure can be maintained in the presence of the net solar heating at low latitudes and net thermal cooling near the poles, if the mesospheric circulation is characterized by rising motion at low latitudes, poleward flow near the mesopause $(\sim 100 \mathrm{~km})$ and subsidence over at high latitudes (Crisp 1986).

Measurements of scattered solar radiation below the clouds (Moroz et al. 1983; Ekonomov et al. 1984) indicate that the atmosphere at these levels is only slightly heated by absorption of sunlight in the weak near-IR $\mathrm{CO}_{2}$ and $\mathrm{H}_{2} \mathrm{O}$ bands and by additional UV absorption. Thermal infrared fluxes and cooling rates in the lower atmosphere are very poorly constrained by the observations and the models. Although Revercomb et al. (1985) managed to correct the Pioneer Venus thermal flux measurements; the error bars are still quite large, such that these results should still be used with caution in thermal balance studies. Spatial variability of the cloud opacity, which can vary as much 
Fig. 36 The altitude-latitude field of the net radiative heating $\left(\mathrm{Kday}^{-1}\right)$ in the Venus mesosphere calculated for the PV OIR thermal structure (from Crisp 1989)

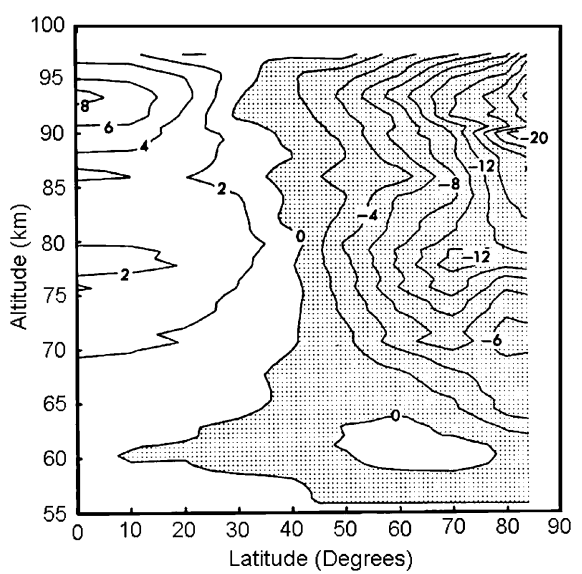

as 20 to 40 optical depths (Fig. 33) can also cause significant variations of thermal radiative forcing at levels within and below the clouds, affecting the local temperatures and heat fluxes (Crisp and Titov 1997). For example, the temperatures measured by the Vega- 1 balloon were systematically $6.5 \mathrm{~K}$ higher than those observed by Vega- 2 at similar levels within the middle cloud region at equatorial latitudes (Linkin et al. 1986b; Crisp et al. 1990). The amplitude of this zonal temperature contrast was surprising because it is almost as large as the pole to equator gradient at these levels. This phenomenon can be explained if the Vega-1 balloon flew in a denser cloud that was heated more strongly as it absorbed upwelling thermal flux from the deep atmosphere (Crisp and Titov 1997).

\subsection{Radiative Forcing of the Atmospheric Circulation}

Remote sensing by the Pioneer Venus, Venera-15, and the Venus Express spacecraft revealed significant latitudinal variations in the mesospheric temperature and cloud structure (Taylor et al. 1980; Lellouch et al. 1997; Zasova et al. 2007b; Grassi et al. 2010; Tellmann et al. 2009). In particular, both the cloud top altitude and aerosol scale height at the cloud tops decreased from the Equator to the Pole (Ignatiev et al. 2009; Lee et al. 2012), with simultaneous development of a strong temperature inversion in the "cold collar" at $60-70^{\circ}$ in both hemispheres (Figs. 4, 5 and 6).

Early estimates of the net radiative forcing have been combined with measurements of the anomalous thermal structure to infer several interesting features of the mesospheric dynamics (Crisp 1986, 1989; Haus and Goering 1990; Titov 1995; Crisp and Titov 1997). First, radiative transfer models confirmed that the mesospheric temperature contrasts between Equator and Pole indicate that these regions are in strong radiative disequilibrium. Radiative energy transport would have quickly destroyed this feature. Its persistence therefore implies significant meridional heat transport by the global circulation. The meridional circulation could produce adiabatic cooling in its rising branch at low latitudes and compression heating in the descending polar branch. Crisp (1989) found that mean subsidence velocities of $\sim 1 \mathrm{~cm} \mathrm{~s}^{-1}$ were enough to produce adiabatic heating, compensating for radiative cooling.

Measurements of the mesospheric temperature structure have been used to infer the zonal circulation at these levels (Lellouch et al. 1997; Zasova et al. 2007b; Piccialli et al. 2008, 2011) and to correlate it with the net radiative heating pattern. If the zonal winds in the mesosphere remain in cyclostrophic balance, the observed temperature structure indicates 
Fig. 37 Globally mean budgets of energy $E$ and entropy $(S=E / T)$ on Venus. The numbers separated by slashes are energy in $\mathrm{W} \mathrm{m}^{-2}$ and entropy in $\mathrm{mW} \mathrm{m}{ }^{-2} \mathrm{~K}^{-1}$

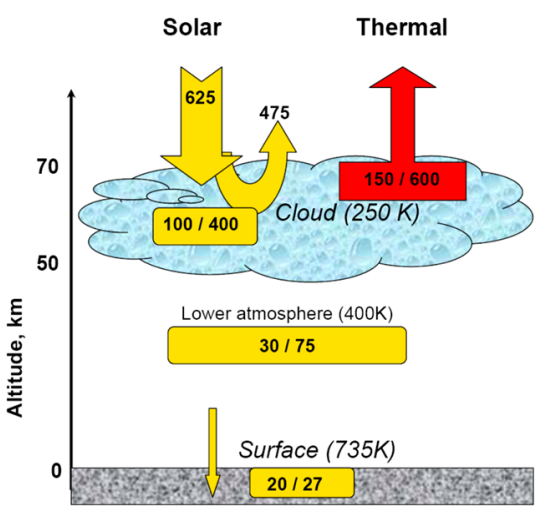

that the superrotating cloud top winds should decay with altitude. It is consistent with the presence of a strong jet stream with wind velocities of $120-140 \mathrm{~m} \mathrm{~s}^{-1}$ near the cloud tops at mid-latitudes $\left(50-60^{\circ}\right)$ that have been seen from cloud tracking (Limaye and Suomi 1981; Khatuntsev et al. 2017; Seiff 1982).

Pioneer Venus OIR and Venera-15 observations indicated the existence of a solar-locked component in the mesospheric temperature field and the diurnal and semi-diurnal thermal tides (Taylor et al. 1983; Zasova et al. 2007b). The peak velocity of the mid-latitude, cloudtop thermal wind jet was found to vary by $10-20 \%$ in correlation with local solar time, thus indicating the importance of radiative forcing. Theoretical and numerical studies showed that thermal tides play an important role in the maintenance of strong zonal winds in the mesosphere (Forbes 2004).

The third peculiarity of the Venus radiative balance that has implications for atmospheric dynamics is the vertical distribution of radiative heating. As noted above, on Venus, most of the solar energy is deposited in the upper cloud $(>57 \mathrm{~km})$ rather than at the surface. This makes Venus a special case among terrestrial planets because its atmosphere is heated from the top while the atmospheres of Mars and Earth receive most of their solar energy at the surface.

\subsection{Global Balance of Radiative Entropy}

Radiation carries not only energy but also entropy. Its flux is proportional to that of the radiative flux divided by the temperature at which energy is deposited or emitted. Since heating by solar radiation and cooling by thermal emission occur at different altitudes and temperatures, planets have a non-zero (usually negative) balance of radiative entropy. This flux is balanced by the entropy production in the irreversible (dissipative) processes such as viscous and turbulent dissipation, phase transitions, and precipitation. The radiative entropy flux can be estimated from the radiative energy balance and is a measure of the cumulative effect of all dissipative processes on a planet.

Figure 37 shows a sketch of the global mean budgets of energy and entropy on the planet, which is also discussed by Schubert and Mitchell (2013). At the cloud level, Venus receives $\sim 400 \mathrm{~mW} \mathrm{~m}^{-2} \mathrm{~K}^{-1}$ of entropy from solar radiation and loses $\sim 600 \mathrm{~mW} \mathrm{~m}^{-2} \mathrm{~K}^{-1}$ from thermal emission. A relatively small amount of energy and entropy is delivered to the lower atmosphere and the surface by the solar flux (Fig. 37). The net global mean budget of radiative entropy on Venus is about $-100 \mathrm{~mW} \mathrm{~m}^{-2} \mathrm{~K}^{-1}$. 
Table 2 Comparison of sources and sinks of planetary entropy for Venus and Earth

\begin{tabular}{lll}
\hline Quantity & Earth, $\mathrm{mW} \mathrm{m}^{-2} \mathrm{~K}^{-1}$ (Goody 2000) & Venus, $\mathrm{mW} \mathrm{m}^{-2} \mathrm{~K}^{-1}$ (Titov et al. 2007) \\
\hline Net radiative sink & -70 & -100 \\
Moist convection & +55 & 0 \\
Mechanical dissipation & +12 & $\sim 1$ \\
Net balance & -3 & -100 \\
\hline
\end{tabular}

Comparison of the entropy budget on Venus with that on Earth can shed light on the differences in irreversible processes on both planets (Table 2). Goody (2000) assessed their effect on Earth, finding the total mean flux of radiative entropy on Earth is about $-70 \mathrm{~mW} \mathrm{~m}{ }^{-2} \mathrm{~K}^{-1}$. Viscous and turbulent dissipation, water phase transitions and precipitation were found to be the main sources of entropy on Earth (Goody 2000; Renno 2001). The Venus dry climate suggests a negligible role of the dissipative mechanisms associated with transport, phase transitions of water and precipitation. Observations suggest rather weak turbulence within the cloud layer with an eddy diffusion coefficient of $10^{4}-10^{3} \mathrm{~cm}^{2} \mathrm{~s}^{-1}$ (Kerzhanovich and Marov 1983) and corresponding entropy production not exceeding $1 \mathrm{~mW} \mathrm{~m}^{-2} \mathrm{~K}^{-1}$. Thus, the entropy sources and sinks on Earth are quite different from those on Venus. However, the role of large scale dynamics, chemical reactions, and cloud processes on the Venusian entropy budget still needs to be studied.

Another difference between the entropy balances on Earth and Venus is the vertical distribution of the radiative sinks and sources of entropy. Earth receives solar energy and entropy mainly at the surface and emits them to space mostly from the atmosphere (Renno and Ingersoll 1996; Renno 1997; Goody 2000). On Venus, the sources of radiative entropy at the surface and in the lower atmosphere represent only $20 \%$ of the total balance (Titov et al. 2007). The largest sinks and sources of radiative entropy on Venus are both located in the upper cloud deck.

General principles of dynamical systems when applied to the Venus climate can lead to interesting, although so far only qualitative, conclusions. Nonlinear dynamics shows that the emergence of higher levels of order in dissipative systems that exchange energy and entropy with environment is typical of both hydrodynamics (e.g., Rayleigh-Benard convection) and chemistry (e.g. Zhabotinsky reaction) (Prigogine 1980). Strong external forcing can push such systems to a stationary state which is far from thermodynamic equilibrium and which is characterized by the maximum entropy production (MEP). The MEP principle was applied to planetary atmospheres and successfully predicted such general parameters of the Earth climate as surface temperature, cloud coverage, meridional energy transport (Paltridge 1975; Grassl 1990) and Equator-to-Pole temperature contrasts (Lorenz et al. 2001). Venus gives another example of such systems. The general circulation is in a steady but strongly nonequilibrium state. The super-rotating Venusian atmosphere (Gierasch et al. 1997) has a rather high level of organization, and thus, low entropy maintained by energy and entropy exchange with the Sun and space.

\subsection{Greenhouse Effect and Climate Evolution}

The planets receive solar energy in the ultraviolet, visible, and near-infrared spectral ranges, in which their atmospheres are relatively transparent. The surface and lower atmosphere get rid of the absorbed energy by emitting radiation at infrared wavelengths. Strong absorption bands of gases and aerosols trap the heat in the lower atmosphere that results in raising 
Table 3 The effect of the removal of infrared opacity sources on the surface temperature (from Titov et al. 2007)

\begin{tabular}{ll}
\hline Species & Change in surface temperature, $\mathbf{K}$ \\
\hline $\mathrm{CO}_{2}$ & -420 \\
Clouds & -140 \\
$\mathrm{H}_{2} \mathrm{O}$ & -70 \\
$\mathrm{OCS}$ & -12 \\
$\mathrm{CO}$ & -3 \\
$\mathrm{SO}_{2}$ & -3 \\
$\mathrm{HCl}$ & -2 \\
\hline
\end{tabular}

the surface temperature. This process is referred to as the atmospheric greenhouse effect. The larger the infrared opacity, the higher the surface temperature required to balance the incoming solar flux. The difference between the actual surface temperature and the effective temperature of the planet without an atmosphere is a measure of the greenhouse effect. Its value reaches $\sim 500 \mathrm{~K}$ on Venus, which is by far the largest of the terrestrial planets.

Bullock and Grinspoon (2001) used a one-dimensional, two-stream radiative-convective equilibrium (RCE) model to calculate the globally averaged temperature structure of the Venus atmosphere, to study its sensitivity to various model parameters, and possible evolution with time. Gaseous opacities were obtained from the correlated-k coefficients (Goody et al. 1989) for $\mathrm{CO}_{2}$ and the eight radiatively active trace gases (Fig. 31) with line parameters taken from the HITRAN (Rothman et al. 1998, 2005) and HITEMP (Wattson and Rothman 1992) spectral databases. $\mathrm{CO}_{2}$ (Moskalenko et al. 1979) and $\mathrm{H}_{2} \mathrm{O}$ continuum opacities (Liou 1992) were included, as were Rayleigh scattering by $\mathrm{CO}_{2}$ and $\mathrm{N}_{2}$ (Van de Hulst 1981). Absorption and scattering in the clouds was calculated assuming spherical particles in the globally averaged cloud model based on the Pioneer Venus measurements (Knollenberg and Hunten 1980).

The lower atmosphere is opaque at infrared wavelengths, with $\mathrm{CO}_{2}$ being the principal source of absorption at wavelengths near 2.0, 2.7, 4.3, 4.8, 5.2, and $15 \mu \mathrm{m}$. Water vapor, $\mathrm{SO}_{2}, \mathrm{CO}$, and $\mathrm{H}_{2} \mathrm{SO}_{4}$ clouds provide the primary sources of opacity between these bands, thus strongly affecting the energy balance of the atmosphere. $\mathrm{H}_{2} \mathrm{O}$ also absorbs in the 2.2 to $2.5 \mu \mathrm{m}$ range. Furthermore, the water vapor detected at this wavelength is from just below the clouds, while $\mathrm{H}_{2} \mathrm{O}$ absorption at $1-1.2 \mu \mathrm{m}$ comes from the atmosphere immediately above the surface. OCS also absorbs in the 2.2-2.5 $\mu \mathrm{m}$ range, and has been observed (Pollack et al. 1993; Arney et al. 2014). The most significant windows between $\mathrm{CO}_{2}$ bands are between 1-1.2 $\mu \mathrm{m}$ where only $\mathrm{H}_{2} \mathrm{O}$ absorbs, at $1.7 \mu \mathrm{m}$ where $\mathrm{HCl}$ and $\mathrm{CO}$ are also detectable, and between 2.2 and $2.5 \mu \mathrm{m}$, where absorption by $\mathrm{CO}$ and $\mathrm{SO}_{2}$ may be seen. Because absorption by these species occurs between bands of the major absorber, $\mathrm{CO}_{2}$, changes in their abundance have a large, disproportionate influence on the Venus greenhouse effect. For instance, the water vapor mixing ratio on average is only $30 \mathrm{ppm}$, but it contributes about $70 \mathrm{~K}$ to the greenhouse effect due to absorption bands at $1.4,1.9$, and $2.5 \mu \mathrm{m}$. Therefore, natural variations of the atmospheric water abundance have a significant effect on the surface temperature. To a lesser extent than water, perturbations to atmospheric sulfur gas abundance can be expected to alter the efficiency of the Venus greenhouse effect.

By subtracting one atmospheric absorber at a time and re-calculating the thermal fluxes and the equilibrium state of the atmosphere, we obtain the contribution of each constituent to the greenhouse effect (Table 3).

Contributions of the atmospheric components are not additive because of strong overlap between the absorption bands. Thus, the cumulative contribution of all opacity sources in 
Table 3 exceeds the observed greenhouse effect. These numerical experiments only take into account changes in the atmospheric opacity due to removal of each trace gas, but neglect their effect on cloud formation. For instance, removal of sulfur dioxide as an optical agent from the model only marginally affects the greenhouse effect (Table 3) while actual removal of this gas from the atmosphere would cause the disappearance of the clouds. This would in turn reduce the greenhouse effect due to infrared scattering within the clouds, along with a decrease in the planetary albedo. The implications of this kind of change for the surface temperature, with competing albedo and greenhouse forcing, is not obvious without detailed calculations. The surface temperature sensitivity of a fully coupled radiative/convective and cloud microphysical model to the variations in abundances of water vapor and sulfur dioxide is discussed in detail by Bullock et al. (2007).

The greenhouse effect also acts on Mars and Earth, although it results in less dramatic changes in surface temperature. On both planets, it is mainly due to the presence of $\mathrm{H}_{2} \mathrm{O}$ and $\mathrm{CO}_{2}$ in the atmosphere and reaches a few degrees on Mars and 30-40 K on Earth. Although the greenhouse effect on our planet is rather moderate, it is obviously responsible for maintaining surface temperature above the freezing point and climate conditions comfortable for life. Without the greenhouse effect the mean temperature would fall to $\sim-20^{\circ} \mathrm{C}$, oceans would freeze, and life would become impossible. Since the conditions on the terrestrial planets and especially the amount of $\mathrm{CO}_{2}$ and $\mathrm{H}_{2} \mathrm{O}$ in the atmosphere could have varied over geological history, the greenhouse effect could have played a more important role in the past (Kasting 1988).

The greenhouse effect seems to have played an important role in the evolution of the atmospheres on all terrestrial planets and, in particular, on Venus (Ingersoll 1969; Pollack 1971, Rasool and de Bergh 1970). Theories about the planets formation suggest that the neighboring planets Earth, Mars and Venus received similar amounts of water during their formation (Ip and Fernandez 1988). However, the atmosphere of Venus currently has about 100,000 times less water than the oceans and atmosphere of the Earth and is strongly enriched in deuterium. The measurements on Venus give $D / H=150 \pm 30$ (Donahue et al. 1982; de Bergh et al. 2006) and up to $240 \pm 25$ (Fedorova et al. 2008) of the terrestrial value. This suggests that Venus lost most of its water at some time in the past. The efficiency of escape depends strongly on the amount of hydrogen and deuterium in the upper atmosphere. The important result of the radiative-convective models of the early Venus atmosphere is that the greenhouse mechanism efficiently forces water into the stratosphere, where solar UV photons readily dissociate $\mathrm{H}_{2} \mathrm{O}$ molecules, thus creating favorable conditions for hydrogen escape. The hydrodynamic escape is rapid enough to eliminate a large ocean in less than 600 million years (Kasting et al. 1984; Kasting 1988).

The current Venus climate is a system with a complex feedback between radiative effects, cloud formations processes, and surface-atmosphere interactions with water vapor, with sulfur dioxide playing the key role in this balance. Bullock and Grinspoon (2001) used their RCE model to study sensitivity of the Venus climate to perturbations in these species due to a global resurfacing event that is assumed to have happened several hundred millions years ago (McKinnon et al. 1997). The Venus climate appears to be stable within a broad range of $\mathrm{H}_{2} \mathrm{O}$ mixing ratios. However, at about 50 times the current abundance, the enhanced water greenhouse begins to warm the atmosphere, evaporating and thinning the clouds from below. This reduces the albedo, further warming the atmosphere, and further evaporating the clouds. This positive feedback destroys the massive $\mathrm{H}_{2} \mathrm{SO}_{4} / \mathrm{H}_{2} \mathrm{O}$ clouds and replaces them with thin, high water-rich clouds, increasing the surface temperature to about $920 \mathrm{~K}$. Further raising of the atmospheric $\mathrm{H}_{2} \mathrm{O}$ content does not increase surface temperatures substantially, 
primarily because it does not enhance the greenhouse effect as much as before, and also because the high water clouds become thicker and more reflective, offsetting the greenhouse effect. Thus, the Venusian climate reaches equilibrium with surface temperatures limited to about $920 \mathrm{~K}$, over a wide range of atmospheric water abundance. If either $\mathrm{H}_{2} \mathrm{O}$ or $\mathrm{SO}_{2}$ abundance fall below $0.5 \mathrm{ppm}$ the clouds also disappear but the surface temperature stabilizes at $\sim 700 \mathrm{~K}$ (Bullock et al. 2007).

\section{Open Issues, Knowledge Gaps and Directions for Future}

\subsection{Thermospheric Temperature and Densities}

The large variability seen in temperatures from SOIR at the terminators and at other local times from SPICAV above $\sim 100 \mathrm{~km}$ is not easy to reconcile with the very small variability seen below $\sim 80 \mathrm{~km}$ from VeRa profiles. Although the Venus Express drag measurements do show a larger variability from the limited observations made during the nominal mission and during the aerobraking experiment, the variation is still considerably smaller than what is seen from SPICAV and SOIR results. One key difference between the experiments is that the drag results yield total atmospheric density whereas both SOIR and SPICAV results are inferred from the results of the $\mathrm{CO}_{2}$ number densities retrieved from the altitude dependence of the spectral values. VeRa results are obtained differently, where neutral number densities are inferred from refractive index profiles assuming a constant atmospheric composition. The SPICAV and SOIR inferences of the total density rely on an assumption of spherical symmetry, i.e. constant molecular weight with altitude. This may not be valid as suggested by the results from the Neutral Mass Spectrometer results on the Pioneer Venus orbiter (Niemann et al. 1980; Seiff et al. 1980) which indicate more than two orders of magnitude change across the terminators in the $\mathrm{CO}_{2}$ number density at $170 \mathrm{~km}$ altitude. The atomic oxygen number densities also vary by almost two orders of magnitude, but the rate of change appears to be different at the morning and evening terminators. Additional measurements at different zenith angles and modelling is needed to understand the atmospheric density, not just at higher altitudes, but also in the troposphere where the atmosphere does not appear to be well mixed in view of the vertical gradient of mean molecular weight.

\subsection{Absorbers of Solar Radiation in the Clouds and Cloud Top Temperatures}

The clouds make Venus the most reflective planet in the Solar System, and hence the question arises whether Venus has indeed become more reflective since the older measurements, when changes in the Venus cloud cover and the $\mathrm{SO}_{2}$ abundance above the cloud tops have been observed. Deposition of incident solar energy is the primary process that determines the atmospheric temperature and is modulated by photochemical and physical (phase) change processes. We have learned that unknown absorbers are responsible for almost half of the absorbed solar energy in the ultraviolet region and other absorbers may contribute between 400-900 nm with some absorption of near infrared radiation by the sulfuric acid droplets emitted by the surface and deeper atmosphere. One may then expect that within the clouds where the ultraviolet absorber is present, one would find higher temperatures, especially near the cloud tops. Confirming this with observations has not yet been possible. The problem is that spatial measurements of cloud top temperatures have not yet been possible with high spatial resolution. 
Fig. 38 (Top) Day-to-day variations of residual patterns at $8.66 \mu \mathrm{m}$ after high-pass filtering. The lower-right image illustrates the dayside (white) and the nightside (gray) of Venus on October 25, 2007.

Latitude-longitude coordinates at $30^{\circ}$ intervals are shown on this image (from Sato et al. 2014). (Bottom) Akatsuki's longwave infrared imager (LIR) takes images in the $8-12 \mu \mathrm{m}$ range revealing cloud-top temperatures. The left image was acquired by Akatsuki about 5 hours after Venus arrival (December 7, 2015 at 05:24 UT) and captured the planet wide standing gravity wave (Fukuhara et al. 2017) straddling the equator from a distance of 72,000 kilometers (JAXA). The image on the right was obtained at a later time and longitude showing the cold collar in the north and the lower cloud tops (warmer brightness temperatures) in the polar region and very low thermal contrasts $(<2 \mathrm{~K})$ over low and mid latitudes. Akatsuki images are from JAXA
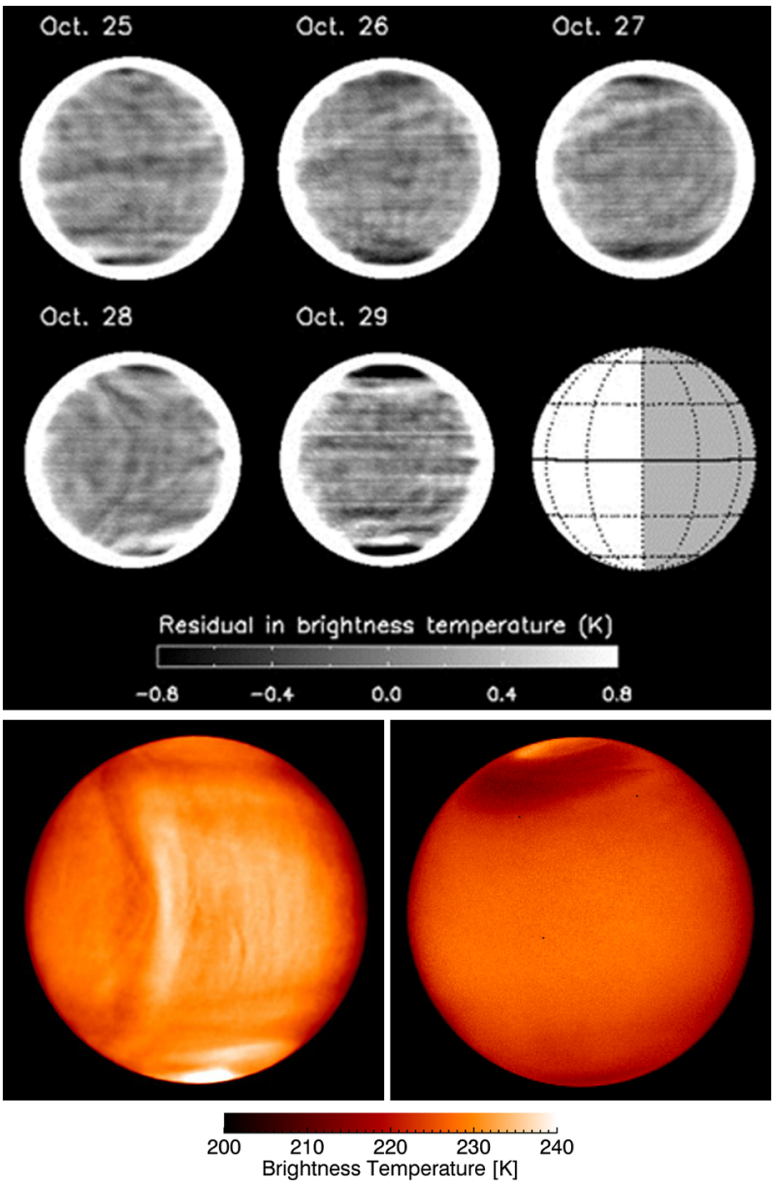

The Akatsuki orbiter launched by Japan in May 2010 (Nakamura et al. 2007, 2014, 2016) is beginning to investigate this relationship after a successful orbital insertion around Venus on 7 December 2015, with routine observations beginning in April 2016. The first image of Venus obtained from orbit from the LIR camera (Fukuhara et al. 2011) in the 8-12 $\mu \mathrm{m}$ wavelengths shows small scale temperature detail (Fig. 38) which has been previously seen in high resolution thermal infrared images from the Subaru telescope (Sato et al. 2014). The relatively small scale thermal contrasts seen in the Subaru data are of the order of $\sim 1 \mathrm{~K}$ and are variable from day to day (Fig. 38, top). The LIR observations from the LIR camera on Akatsuki (Fig. 38, bottom) appear to be comparable. Confirmation awaits additional analysis and observations. The local scale could be indicative of the convection at cloud tops triggered by increased heating due to the inhomogeneously distributed ultraviolet absorber.

Titov et al. (2018) discuss only the inorganic ultraviolet absorbers, but it is apparent that available observations of cloud particle size and chemical composition cannot exclude microorganism absorbers at ultraviolet and longer wavelengths (Limaye et al. 2018b). The nature and identity of the absorbers of sunlight needs to be established. At the same time, causes of spatial and temporal variations of the cloud opacity on the night side also need to be understood. There is a need to establish the average albedo of Venus more accurately to 
Table 4 Altitude on Venus where key atmospheric gases become supercritical

\begin{tabular}{lllll}
\hline Gas & $\begin{array}{l}\text { Critical pressure } \\
(\text { bar })\end{array}$ & $\begin{array}{l}\text { Critical temperature } \\
(\mathrm{K})\end{array}$ & $\begin{array}{l}\text { Altitude on Venus } \\
(\mathrm{km})\end{array}$ & $\begin{array}{l}\text { Venus atmospheric } \\
\text { temperature }(\mathrm{K})\end{array}$ \\
\hline Carbon dioxide & 73.9 & 304.25 & 2.96 & 707 \\
Nitrogen & 34.0 & 126.2 & 14.5 & 622 \\
Carbon monoxide & 34.9 & 132.9 & 14.0 & 716 \\
Sulfur dioxide & 77.8 & 430.7 & 2.15 & - \\
Water & 221.0 & 647.3 & - & 732 \\
Sulfur monoxide & 91.75 & 421.1 & 0.1 & \\
\hline
\end{tabular}

detect changes over annual and longer time scales to understand better the temporal changes in the heating distribution and circulation. Measurements in the cloud layer are needed to distinguish between inorganic and microorganism absorbers. Global abundances of the absorbers would be helpful in understanding the changes in the global cloud cover contrasts.

\subsection{Vertical Gradient of Atmospheric Composition}

The composition of the Venus atmosphere was reviewed by von Zahn and Moroz (1985) and the abundance of the two primary constituents has been generally accepted to be $96.5 \%$ $\mathrm{CO}_{2}$ and $3.5 \% \mathrm{~N}_{2}$. The experimental values obtained by Oyama et al. (1980) however show that the abundance of $\mathrm{N}_{2}$ (measured with an accuracy of $0.01 \%$ ) decreased by about $1.4 \%$ between 52 and $22 \mathrm{~km}$ altitude at the Pioneer Venus Large Probe location. At that time, no explanation for the variation in the nitrogen abundance was available (von Zahn et al. 1983 ) as the impact of the supercritical state of the two primary constituents was not appreciated and considered regarding these results. This gradient was noted by others (von Zahn et al. 1983) but for lack of a plausible explanation even though the measurements were the most accurate ever obtained, the result was ignored and only an average value was provided in the VIRA model, with an error estimate of $0.8 \%$ equal to the standard deviation of the three measurements which is much larger than the accuracy of the nitrogen measurements $\left(<0.02 \%\right.$, Oyama et al. 1980). It is interesting to note that at the same altitudes, $\mathrm{CO}_{2}$ abundances were correspondingly lower (Fig. 39). If the gradient were to extend to the surface as Lebonnois and Schubert (2017) propose to address the unstable layer between 0-7 km and to higher altitudes, the composition of the Venus atmosphere above $52 \mathrm{~km}$ would have noticeably more nitrogen. This is strongly suggested by the recent inference about the nitrogen abundance $(5.38 \pm 0.29 \%$ by volume) at $64 \mathrm{~km}$ altitude from analysis of the Neutron Spectrometer data obtained during the MESSENGER fly-by of Venus in June 2016 (Peplowski and Lawrence 2016).

Table 4 lists the critical pressures and temperature and the approximate altitude where the two major constituents and some key trace gases become supercritical. It has only recently become clear that mixtures of supercritical fluids do not behave like normal fluids. Hendry et al. (2013) report a concentration gradient in a laboratory experiment conducted with a mixture of supercritical nitrogen and carbon dioxide, and cautions that the gradient between 54 and $22 \mathrm{~km}$ altitude reported by Oyama et al. should not be ignored (as done by von Zahn et al. 1983) but confirmed from future missions. This is bolstered by the recent inference of nitrogen abundance $(5.5 \%)$ from analysis of the MESSENGER Neutron Spectrometer data at $64 \mathrm{~km}$ altitude (Peplowski and Lawrence 2016). 
Fig. 39 Altitude profiles of $\mathrm{CO}_{2}$ and $\mathrm{N}_{2}$ volume mixing ratios measured by Oyama et al. (1980) and inferred by Peplowski and Lawrence (2016). The dotted curve shows a linear fit to the nitrogen abundances measured at 64, 52, 41 and $22 \mathrm{~km}$. Error bars represent three standard deviations from average values for $\mathrm{CO}_{2}$. The corresponding values for nitrogen are too small to be seen in this figure. The green curve shows the $\mathrm{CO}_{2}+\mathrm{N}_{2}$ abundance, which is almost $100 \%$ at the three altitudes, sampled

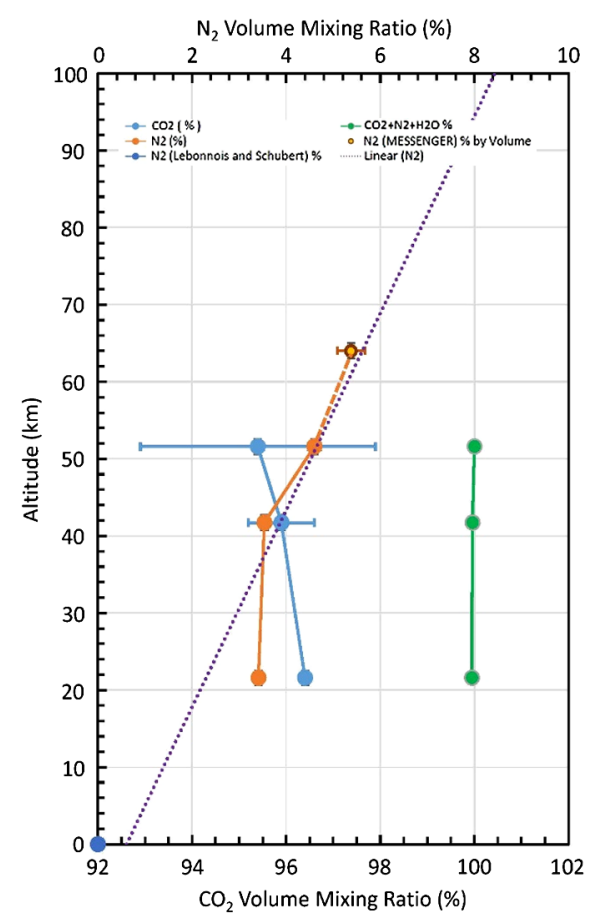

Numerical simulations of the behavior of the mixture of real gas supercritical fluids from current models for the equation of state for real gas mixtures also show some small discrepancies (Goos et al. 2011). Thus, experimental verification of properties of real gas mixtures of the two primary constituents of the Venus atmosphere found in the lower few $\mathrm{km}$ of the Venus atmosphere is critical. A vertical gradient of the mean molecular weight of the Venus atmosphere from surface to $64 \mathrm{~km}$ and higher will impact ALL available vertical profiles either in altitude (all entry probe data) or pressure (all occultations). This is because altitude is the primary variable determined from occultation geometry calculation of the atmospheric pressure estimate requires use of the hydrostatic law. For entry probe data such as Pioneer Large and Small probes, the hydrostatic law is required for pressure and altitude calculations (Seiff et al. 1980). Interestingly, although the Pioneer probes were tracked using DLBI (Counselman et al. 1980), there appear to be no estimates of the distance of the falling probes relative to the planet, which should have been possible from the DLBI data. A comparison of the tracking derived altitudes with the Seiff et al. (1980) values would have revealed a discrepancy due to the constant molecular weight assumption. The confirmation of the vertical gradient in the abundance of nitrogen to higher altitudes is critical.

The expectation is that the atmosphere should be well mixed in primary constituents for all of the vertical structure investigations of the Venus neutral atmosphere below $120 \mathrm{~km}$. If a vertical gradient exists in the $\mathrm{N}_{2}$ abundance at altitudes where the atmosphere should be well mixed, then it is likely that the mean molecular weight assumed to be constant below $120 \mathrm{~km}$ may be variable with altitude. This is another instance of variable mean molecular weight at altitudes above $100 \mathrm{~km}$. In-situ measurements of the $\mathrm{CO}_{2}$ density by the Pioneer Venus ONMS (von Zahn et al. 1983) show that the values change by more than an order of magnitude within one hour of the evening and morning terminator (Fig. 40), which is equivalent to a horizontal distance of about $1600 \mathrm{~km}$ at $100 \mathrm{~km}$ altitude, while the slant path 

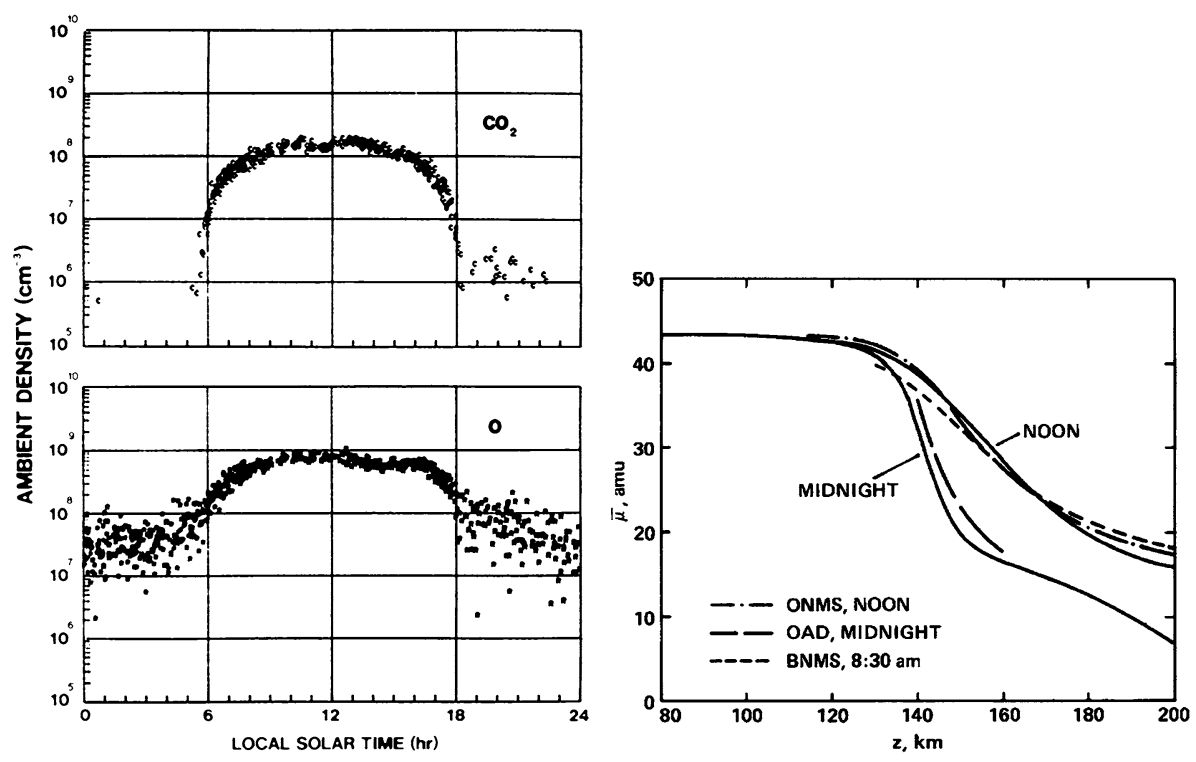

Fig. 40 Left - carbon dioxide (top) and atomic oxygen (bottom) number density measured from Pioneer Venus Orbiter Neutral Mass Spectrometer (ONMS) at $170 \mathrm{~km}$ altitude as a function of local solar time over three diurnal cycles (von Zahn et al. 1983). Right - Altitude profile of mean molecular weight derived from the species profiles from PV ONMS reported by Niemann et al. 1980 showing sharp changes across the terminators. The long-dash curve is from a model used for interpretation of the data (figure from Seiff and Kirk 1982)

for occultation profiles can be several hundred $\mathrm{km}$ long, incurring noticeable change in the local time.

This is particularly relevant for the retrieval of total atmospheric densities from the $\mathrm{CO}_{2}$ densities deduced from the SOIR and SPICAV experiments. Both investigations used a spherical atmosphere assumption with constant mean molecular weight with solar zenith angle (local solar time) and altitude. The impact of the possible vertical gradient of mean molecular weight on temperature profiles from not just SPICAV and SOIR, but all investigations should be investigated, along with experiments to measure the bulk composition from surface to the $140 \mathrm{~km}$ altitude to validate the composition gradient.

\subsection{Near Surface Unstable Layer}

The VeGa 2 lander measured the atmospheric profile on the night side (Sub-solar location: $2.5^{\circ} \mathrm{N} 15.73^{\circ} \mathrm{E}$ longitude) near midnight. Yet, the atmospheric profile shows a thick unstable layer slightly elevated above the surface and above a layer with a less unstable lapse rate. This is a somewhat surprising environmental state on the night side. Given the very long radiative time constant in the deep atmosphere, the unstable layer can arise only in the presence of, (i) the layer being in contact with a hotter surface, raising the question of whether internal heat is responsible, (ii) dynamical processes, or (iii) spurious due to a vertical gradient of the mean molecular weight of the Venus atmosphere due to increasing abundance of nitrogen from zero percent at the surface to higher values above. Lebonnois and Schubert (2017) considered such a gradient only for the unstable layer below $7 \mathrm{~km}$ altitude, but it is possible that the gradient extends to $64 \mathrm{~km}$ and above. 


\subsection{Radiative Balance}

It is unfortunate that Venus albedo has not been measured routinely in the last few decades. Recently Mallama et al. (2006) used ground based day time CCD photometry and SOHO data to increase the phase angle coverage and found higher values for spherical albedo is higher than the older values. But, the impact on the integrated albedo is unclear due to differences in the spectral coverage. As Pérez-Hoyos et al. (2017) point out, the absorption spectrum and the vertical distribution of the need to be measured better for improving the radiative balance knowledge. Identification, biological/chemical origins and properties of likely multiple absorbers in the Venus atmosphere of insolation and their contributions to heating is one area for future efforts.

Previous investigations of radiation in the Venus atmosphere provided a general understanding of the distribution of fluxes, sources and sinks of radiative energy, and of the radiative forcing of the atmospheric dynamics and climate. At the same time, they left a great number of unsolved problems. One of the most important open issues in this field is the variability of atmospheric properties such as the abundance of radiatively active gases, cloud microphysical and optical properties and total opacity, and the influence of these on the energy balance. The second problem concerns the radiative forcing of the atmospheric global circulation. How does the distribution of the sources and sinks of radiative energy influence the atmospheric dynamics? The thermodynamics of the Venus atmosphere, especially regarding mixtures of gases with different supercritical points is the third issue requiring clarification and quantification. New studies of the fluxes and balance of energy and entropy, and the role of dissipative processes and efficiency of the Venus heat engine will help us to understand the Venus climate-controlling mechanisms. The fourth open question concerns the role of radiation in the evolution of the Venus atmosphere, the early greenhouse effect and the loss of water from the planet, as well as more recent climate perturbations during global resurfacing. Resolving these open issues in Venus physics would result in significant progress in comparative planetology and climatology of the terrestrial planets in general, and in the study of the Earth's climate evolution in particular. Future work will require a combination of new observations, laboratory studies, numerical modeling, and theoretical investigations. Remote sensing of the Venus mesosphere will provide the global temperature and aerosol structure, distribution and variations of the radiatively active gases, and characterize the aerosol properties and total cloud opacity with complete latitude and local time coverage. Observations of the night side in the near-IR transparency "windows" from orbit will provide access to the composition and cloud properties in the middle and lower atmosphere regions that had so far only been reached by descent probes. Measurements of the reflected solar and outgoing thermal fluxes from orbit are required to quantify the details of energy and entropy budgets. While the remote sensing observations from orbit provide global coverage, the details of the atmospheric properties, and especially their vertical distribution, require in - situ measurements. The first priority here is the characterization of the aerosol population, including number density, particle size distribution, chemical composition, and optical properties as well as consider possible chemical/biologic origins. Special attention should be paid to the identification of the unknown absorbers of sunlight, due to its important role in the radiative balance. Accurate measurements of the global vertical profiles of the radiatively active gases and their spatial variations are important for development of Venus greenhouse models. Vertical profiles of the radiative heat fluxes are poorly known and new, in - situ observations are required. Finally, precise measurements of the vertical profiles of atmospheric temperatures in the deep atmosphere would constrain models of the Venus greenhouse and help to determine the regions of convective stability. The Venus lower 
atmosphere is an example of an extreme environment. Laboratory measurements of spectral properties of the atmospheric gases at very long path lengths are required. These are difficult to obtain at extreme conditions but are needed to support the analysis of remote and in-situ measurements and state-of-the-art radiative transfer models to study the radiative energy balance and forcing of the global circulation and climate, and their sensitivity to the variation of the atmospheric optical properties.

Acknowledgements SSL acknowledges support from NASA Grants NNX09AE85G and NNX16AC79G. AM acknowledges the FNRS for the Chargé de Recherches position. We appreciate the very helpful and detailed comments from Dr. Mark Bullock and an anonymous reviewer were very useful in refining this manuscript. We are pleased to thank Ms. Rosalyn Pertzborn for careful editing and thoughtful comments. Finally, we appreciate receiving the tabulated data from Dr. Todd Clancy for Fig. 26.

Open Access This article is distributed under the terms of the Creative Commons Attribution 4.0 International License (http://creativecommons.org/licenses/by/4.0/), which permits unrestricted use, distribution, and reproduction in any medium, provided you give appropriate credit to the original author(s) and the source, provide a link to the Creative Commons license, and indicate if changes were made.

\section{References}

D.A. Allen, J.W. Crawford, Cloud structure on the dark side of Venus. Nature 307, 222-224 (1984)

H. Ando, N. Sugimoto, M. Takagi, T. Imamura, Y. Matsuda, The puzzling Venusian polar atmospheric structure reproduced by a general circulation model. Nat. Commun. (2016). https://doi.org/10.1038/ ncomms 10398

A. Arking, Absorption of solar energy in the atmosphere: discrepancy between model and observations. Science 273, 779-782 (1996)

G. Arney, V. Meadows, D. Crisp, S.J. Schmidt, J. Bailey, T. Robinson, Spatially resolved measurements of $\mathrm{H}_{2} \mathrm{O}, \mathrm{HCl}, \mathrm{CO}, \mathrm{OCS}, \mathrm{SO}_{2}$, cloud opacity, and acid concentration in the Venus near-infrared spectral windows. J. Geophys. Res., Planets 119, 1860-1891 (2014)

V.S. Avduevskii, N.F. Borodin, V.N. Vasilev, A.G. Godnev, V.P. Kariagin, V.A. Koverianov, V.M. Kovtunenko, R.S. Kremnev, V.M. Pavlova, M.K. Rozhdestvenskii, Kosm. Issled. 17, 655-660 (1979)

V.S. Avduevskii, A.G. Godnev, V.V. Semenchenko, G.R. Uspenskii, Z.P. Cheremukhina, Investigation of the characteristics of the Venus stratosphere from acceleration measurements during the braking of the Venera-13 and Venera-14 probes. Kosm. Issled. 21, 205-210 (1983a)

V.S. Avduevskii, M.Ia. Marov, N.Iu. Kulikov, V.P. Shari, A.Ia. Gorbachevskii, G.R. Uspenskii, Z.P. Cheremukhina, Structure and parameters of the Venus atmosphere according to Venera probe data, in Venus (University of Arizona Press, Tucson, 1983b), pp. 280-298

V.S. Avduevsky, N.F. Borodin, V.P. Burtsev, Ya.V. Malkov, M.Ya. Marov, S.F. Morozov, M.K. Rozhdestvenskii, R.S. Romanov, S.S. Sokolov, V.G. Fokin, Z.P. Cheremukhina, V.I. Shkirina, Automatic stations Venera 9 and Venera 10: functioning of descent vehicles and measurement of atmospheric parameters. Cosm. Res. 14, 577-586 (1976)

R.D. Baker, G. Schubert, P.W. Jones, Cloud-level penetrative compressible convection in the Venus atmosphere. J. Atmos. Sci. 55, 3-18 (1998)

E.S. Barker, Detection of CS2 in the UV spectrum of Venus. Bull. Am. Astron. Soc. 10, 548 (1978)

E.S. Barker, Detection of SO2 in the UV spectrum of Venus. Geophys. Res. Lett. 6, 117-120 (1979)

E.S. Barker, J.H. Woodman, M.A. Perry, B. Hapke, R. Nelson, Relative spectrophotometry of Venus from 3067 to $5960 \AA$ A. J. Atmos. Sci. 32, 1205-1211 (1975)

J.F. Bell, D. Crisp, P.G. Lucey, T.A. Ozorosky, W.A. Sinton, S.C. Willis, B.A. Campbell, Spectroscopic observations of bright and dark emission features on the night side of Venus. Science 252, 1293-1296 (1991)

J.-L. Bertaux, D. Nevejans, O. Korablev, E. Villard, E. Quémerais, E. Neefs, F. Montmessin, F. Leblanc, J.P. Dubois, E. Dimarellis, A. Hauchecorne, F. Lefevre, P. Rannou, J.Y. Chaufray, M. Cabane, G. Cernogora, G. Souchon, F. Semelina, A. Reberac, E. Van Ransbeek, S. Berkenbosch, R. Clairquin, C. Muller, F. Forget, F. Hourdin, O. Talagrand, A. Rodin, A. Fedorova, A. Stepanov, A. Vinogradov, A. Kiselev, Y. Kalinnikov, G. Durry, B. Sandel, A. Stern, J.-C. Gérard, SPICAV on Venus Express: three spectrometers to study the global structure and composition of the Venus atmosphere. Planet. Space Sci. 55, 1673-1700 (2007a) 
J.-L. Bertaux, A.C. Vandaele, O. Korablev, E. Villard, A. Fedorova, D. Fussen, E. Quémerais, D. Belyaev, A. Mahieux, F. Montmessin, C. Muller, E. Neefs, D. Nevejans, V. Wilquet, J.P. Dubois, A. Hauchecorne, A. Stepanov, I. Vinogradov, A. Rodin, J.L. Bertaux, D. Nevejans, O. Korablev, F. Montmessin, A.C. Vandaele, A. Fedorova, M. Cabane, E. Chassefière, J.Y. Chaufray, E. Dimarellis, J.P. Dubois, A. Hauchecorne, F. Leblanc, F. Lefèvre, P. Rannou, E. Quémerais, E. Villard, D. Fussen, C. Muller, E. Neefs, E. vanRansbeeck, V. Wilquet, A. Rodin, A. Stepanov, I. Vinogradov, L. Zasova, F. Forget, S. Lebonnois, D. Titov, S. Rafkin, G. Durry, J.C. Gérard, B. Sandel, A warm layer in Venus' cryosphere and high-altitude measurements of $\mathrm{HF}, \mathrm{HCl}, \mathrm{H} 2 \mathrm{O}$ and $\mathrm{HDO}$. Nature 450, 646-649 (2007b)

J. Bertaux, I.V. Khatuntsev, A. Hauchecorne, W.J. Markiewicz, E. Marcq, S. Lebonnois, M. Patsaeva, A. Turin, A. Fedorova, Influence of Venus topography on the zonal wind and UV albedo at cloud top level: the role of stationary gravity waves. J. Geophys. Res., Planets 121, 1087-1101 (2016). https://doi.org/10.1002/2015JE004958

B. Bezard, C. de Bergh, D. Crisp, J.-P. Maillard, The deep atmosphere of Venus revealed by high-resolution nightside spectra. Nature 345, 508-511 (1990)

B. Bezard, C.C.C. Tsang, R.W. Carlson, G. Piccioni, E. Marcq, P. Drossart, Water vapor abundance near the surface of Venus from Venus Express/VIRTIS observations. J. Geophys. Res., Planets 114, E00B39 (2009). https://doi.org/10.1029/2008JE003251

B. Bézard, A. Fedorova, J.-L. Bertaux, A. Rodin, O. Korablev, The 1.10- and 1.18- $\mu \mathrm{m}$ nightside windows of Venus observed by SPICAV-IR aboard Venus Express. Icarus 216(1), 173-183 (2011). https://doi.org/10.1016/j.icarus.2011.08.025

R.P. Binzel, Comparative climatology of terrestrial planets, in The University of Arizona Space Science Series, ed. by S.J. Mackwell, A.A. Simon-Miller, J.W. Harder, M.A. Bullock (2013)

J. Blamont, B. Ragent, Further results of the Pioneer Venus nephelometer experiment. Science 205, 67-70 (1979)

R.W. Boese, J.B. Pollack, P.M. Silvaggio, First results from the large probe infrared radiometer experiment. Science 203, 797-800 (1979). https://doi.org/10.1126/science.203.4382.797

S.W. Bougher, R.E. Dickinson, E.C. Ridley, R.G. Roble, A. Nagy, T.E. Cravens, Venus mesosphere and thermosphere, II: global circulation, temperature, and density variations. Icarus 68, 284-312 (1986)

S.W. Bougher, R.E. Dickinson, E.C. Ridley, R.G. Roble, Venus mesosphere and thermosphere, III: threedimensional general circulation with coupled dynamics and composition. Icarus 73, 545-573 (1988)

S.W. Bougher, A. Brecht, R. Schulte, J. Fischer, C. Parkinson, A. Mahieux, V. Wilquet, A.C. Vandaele, Upper atmosphere temperature structure at the venusian terminators: a comparison of SOIR and VTGCM results. Planet. Space Sci. 113-114, 336-346 (2015)

M.A. Bullock, D.H. Grinspoon, The recent evolution of climate on Venus. Icarus 150, 19-37 (2001)

M.A. Bullock, D. Grinspoon, S.C. Solomon, J.W. Head III., R.J. Phillips, Climate change and tectonics on Venus, in Exploring Venus as a Terrestrial Planet, ed. by L.W. Esposito (American Geophysical Union, Washington, 2007)

M.A. Bullock, D.H. Grinspoon, The atmosphere and climate of Venus, in Comparative Climatology of Terrestrial Planets, ed. by S.J. Mackwell, et al. (The University of Arizona Press, Tuscon, 2013)

A. Cardesín Moinelo, G. Piccioni, A. Migliorini, P. Drossart, Global mapping of Venus' Atmosphere using accumulated projections of Virtis Venus Express observations (2008)

R.W. Carlson, Venus's ultraviolet absorber: cyclo-octal (S8) and polymeric sulfur (Sx) and their latitudinal behavior, in International Conference on Venus, Oxford (2016). https://venus2016.files.wordpress.com/ 2016/04/02_carlson_uvabsorber_venus2016_public.pdf

R.W. Carlson, F.W. Taylor, The Galileo encounter with Venus: results of the near-infrared mapping spectrometer. Planet. Space Sci. 41, 475-476 (1993)

R.W. Carlson, L.W. Kamp, K.H. Baines, J.B. Pollack, D.H. Grinspoon, T. Encrenaz, P. Drossart, E. Lellouch, B. Bezard, Variations in Venus cloud particle properties: a new view of Venus's cloud morphology as observed by the Galileo near-infrared mapping spectrometer. Planet. Space Sci. 41, 477-486 (1993)

R.T. Clancy, B.J. Sandor, G. Moriarty-Schieven, Thermal structure and CO distribution for the Venus mesosphere/lower thermosphere: 2001-2009 inferior conjunction sub-millimeter CO absorption line observations. Icarus 217, 779-793 (2012)

R.T. Clancy, B.J. Sandor, Hodge, Doppler winds mapped around the lower thermospheric terminator of Venus: 2012 solar transit observations from the James Clark Maxwell Telescope. Icarus 254(1), 233-258 (2015)

D.L. Coffeen, Venus cloud contrasts, in Planetary Atmospheres, ed. by C. Sagan, T.C. Owen, H.J. Smith (Reidel, Dordrecht, 1971), pp. 84-90

C.C. Counselman, S.A. Gourevitch, R.W. King, G.B. Loriot, E.S. Ginsberg, Zonal and meridional circulation of the lower atmosphere of Venus determined by radio interferometry. J. Geophys. Res. 85, 8026-8030 (1980) 
C. Covey, R.M. Haberle, C.P. McKay, D.V. Titov, A.A. Simon-Miller, J.W. Harder, The greenhouse effect and climate feedbacks, in Comparative Climatology of Terrestrial Planets, ed. by S.J. Mackwell (2013), pp. 163-179

D. Crisp, Radiative forcing of the Venus mesosphere, I: solar fluxes and heating rates. Icarus 67, 484-514 (1986)

D. Crisp, Radiative forcing of the Venus mesosphere, II: thermal fluxes, cooling rates, and radiative equilibrium temperatures. Icarus 77, 391-413 (1989)

D. Crisp, D.V. Titov, The thermal balance of the Venus atmosphere, in Venus-II, ed. by S.W. Bougher, D.M. Hunten, R.J. Phillips (The University of Arizona Press, Tucson, 1997), pp. 353-384

D. Crisp, A.P. Ingersoll, C.E. Hildebrand, R.A. Preston, VEGA balloon meteorological experiments. Adv. Space Res. 10, 109-124 (1990)

D. Crisp, D.A. Allen, D.H. Grinspoon, J.B. Pollack, The dark side of Venus: near-infrared images and spectra from the Anglo-Australian Observatory. Science 253, 1263-1266 (1991a)

D. Crisp, S. McMuldorch, S.K. Stephens, W.M. Sinton, B. Ragent, K.-W. Hodapp, R.G. Probst, L.R. Doyle, D.A. Allen, J. Elias, Ground-based near-infrared imaging observations of Venus during the Galileo encounter. Science 253, 1538-1541 (1991b)

C. de Bergh, B. Bézard, D. Crisp, J.P. Maillard, T. Owen, J. Pollack, D. Grinspoon, Water in the deep atmosphere of Venus from high-resolution spectra of the night side. Adv. Space Res. 15(4), 79-88 (1995)

C. de Bergh, V.I. Moroz, F.W. Taylor, D. Crisp, B. Bézard, L.V. Zasova, The composition of the atmosphere of Venus below 100 km altitude: an overview. Planet. Space Sci. 54, 1389-1397 (2006). https://doi.org/10.1016/j.pss.2006.04.020

T.M. Donahue, J.H. Hoffman, R.R. Hodges Jr., A.J. Watson, Venus was wet: a measurement of the ratio of deuteriumt o hydrogen. Science 216, 630-633 (1982)

P. Drossart, F. Montmessin, The legacy of Venus Express: highlights from the first European planetary mission to Venus. Astron. Astrophys. Rev. 23, 5 (2015). https://doi.org/10.1007/s00159-015-0088-0

P. Drossart, B.Th. Encrenaz, E. Lellouch, E.M. Roos, F.W. Taylor, A.D. Collard, S.B. Calcutt, J.B. Pollack, D.H. Grinspoon, Search for spatial variations of the $\mathrm{H}_{2} \mathrm{O}$ abundance in the lower atmosphere of Venus from NIMS-Galileo. Planet. Space Sci. 41, 495-504 (1993). https://doi.org/10.1016/ 0032-0633(93)90032-W

P. Drossart, G. Piccioni, J.C. Gérard, M.A. Lopez-Valverde, A. Sanchez-Lavega, L. Zasova, R. Hueso, F.W. Taylor, B. Bézard, A. Adriani, F. Angrilli, G. Arnold, K.H. Baines, G. Bellucci, J. Benkhoff, J.P. Bibring, A. Blanco, M.I. Blecka, R.W. Carlson, A. Coradini, A. Di Lellis, T. Encrenaz, S. Erard, S. Fonti, V. Formisano, T. Fouchet, R. Garcia, R. Haus, J. Helbert, N.I. Ignatiev, P. Irwin, Y. Langevin, S. Lebonnois, D. Luz, L. Marinangeli, V. Orofino, A.V. Rodin, M.C. Roos-Serote, B. Saggin, D.M. Stam, D. Titov, G. Visconti, M. Zambelli, C. Tsang, A dynamic upper atmosphere of Venus as revealed by VIRTIS on Venus Express. Nature 450, 641-645 (2007). https://doi.org/10.1038/nature06140

A. Dutt, S.S. Limaye, Adiabatic lapse rate and static stability in the Venus atmosphere calculated from real gas mixture models. arXiv:1806.06835v1 [astro-ph.EP] (2018)

E.P. Ekonomov, V.I. Moroz, B.E. Moshkin, V.I. Gnedykh, Yu.M. Golovin, A.V. Grigoriev, Scattered UV solar radiation within the clouds of Venus. Nature 307, 345-347 (1984)

Th. Encrenaz, E. Lellouch, J. Cernicharo, G. Paubert, S. Gulkis, T. Spilker, The thermal profile and water abundance in the Venus mesosphere from $\mathrm{H}_{2} \mathrm{O}$ and $\mathrm{HDO}$ millimeter observations. Icarus 117, 162-172 (1995). https://doi.org/10.1006/icar.1995.1149

Th. Encrenaz, T.K. Greathouse, M.J. Richter, C. DeWitt, T. Widemann, B. Bézard, T. Fouchet, S.K. Atreya, H. Sagawa, HDO and SO2 thermal mapping on Venus, III: short-term and long-term variations between 2012 and 2016. Astron. Astrophys. 595, A74 (2016)

L.W. Esposito, Ultraviolet contrasts and absorbers near the Venus cloud tops. J. Geophys. Res. 85(AI3), 8151-8157 (1980)

L.W. Esposito, R.G. Knollenberg, M.Y. Marov, O.B. Toon, R.P. Turco, Clouds and hazes of Venus, in Venus (University of Arizona Press, Tucson, 1983), pp. 484-564

J. Farquhar, H. Bao, M. Thiemens, Atmospheric influence of earth's earliest sulfur cycle. Science 289(5480), 756-758 (2000). https://doi.org/10.1126/science.289.5480.756

A. Fedorova, O. Korablev, A.-C. Vandaele, J.-L. Bertaux, D. Belyaev, A. Mahieux, E. Neefs, W.V. Wilquet, R. Drummond, F. Montmessin, E. Villard, $\mathrm{HDO}$ and $\mathrm{H}_{2} \mathrm{O}$ vertical distributions and isotopic ratio in the Venus mesosphere by Solar Occultation at Infrared spectrometer on board Venus Express. J. Geophys. Res. 113, E00B22 (2008). https://doi.org/10.1029/2008JE003146

A. Fedorova, E. Marcq, M. Luginin, O. Korablev, J.-L. Bertaux, F. Montmessin, Variations of water vapor and cloud top altitude in the Venus' mesosphere from SPICAV/VEx observations. Icarus 275, 143-162 (2016). https://doi.org/10.1016/j.icarus.2016.04.010

G. Fjeldbo, A.J. Kliore, R. Von Eshleman, The neutral atmosphere of Venus as studied with the Mariner V radio occultation experiments. Astron. J. 76, 123 (1971) 
J.M. Forbes, Tides in the middle and upper atmospheres of Mars and Venus. Adv. Space Res. 33, 125-131 (2004)

V. Formisano, F. Angrilli, G. Arnold, S. Atreya, K.H. Baines, G. Bellucci, B. Bézard, F. Billebaud, D. Biondi, M.I. Blecka, L. Colangeli, L. Comolli, D. Crisp, M. D’Amore, T. Encrenaz, A. Ekonomov, F. Esposito, C. Fiorenza, S. Fonti, M. Giuranna, D. Grassi, B. Grieger, A. Grigoriev, J. Helbert, H. Hirsch, N. Ignatiev, A. Jurewicz, I. Khatuntsev, S. Lebonnois, E. Lellouch, A. Mattana, A. Maturilli, E. Mencarelli, M. Michalska, J. Lopez Moreno, B. Moshkin, F. Nespoli, Y. Nikolsky, F. Nuccilli, P. Orleanski, E. Palomba, G. Piccioni, M. Rataj, G. Rinaldi, M. Rossi, B. Saggin, D. Stam, D. Titov, G. Visconti, L. Zasova, The planetary Fourier spectrometer (PFS) onboard the European Venus Express mission. Planet. Space Sci. 54(13-14), 1298-1314 (2006)

T. Fukuhara, M. Taguchi, T. Imamura, M. Nakamura, M. Ueno, M. Suzuki, N. Iwagami, M. Sato, K. Mitsuyama, G.L. Hashimoto, R. Ohshima, T. Kouyama, H. i Ando, M. Futaguchi, LIR: Longwave Infrared Camera onboard the Venus orbiter Akatsuki. Earth Planets Space 63, 1009 (2011). https://doi.org/10.5047/eps.2011.06.019

T. Fukuhara, M. Futaguchi, G.L. Hashimoto, T. Horinouchi, T. Imamura, N. Iwagami, T. Kouyama, S. Murakami, M. Nakamura, K. Ogohara, M. Sato, T.M. Sato, M. Suzuki, M. Taguchi, S. Takagi, M. Ueno, S. Watanabe, M. Yamada, A. Yamazaki, Large stationary gravity wave in the atmosphere of Venus. Nat. Geosci. 10, 85-88 (2017). https://doi.org/10.1038/ngeo2873

I. Garate-Lopez, A. García Muñoz, R. Hueso, A. Sánchez-Lavega, Instantaneous three-dimensional thermal structure of the South Polar Vortex of Venus. Icarus 245, 16-31 (2015). https://doi.org/10.1016/ j.icarus.2014.09.030

P. Gierasch, R.M. Goody, R.E. Young, D. Crisp, C. Edwards, R. Kahn, D. McCleeses, D. Rider, A. Del Genio, R. Greely, A. Hou, C.B. Leovy, M. Newman, The general circulation of the Venus atmosphere: an assessment, in Venus-II, ed. by S.W. Bougher, D.M. Hunten, R.J. Phillips (The University of Arizona Press, Tucson, 1997), pp. 459-500

G. Gilli, M.A. López-Valverde, S. Bougher, A. Brecht, P. Drossart, G. Piccioni, Carbon monoxide and temperature in the upper atmosphere of Venus from VIRTIS/Venus Express non-LTE limb measurements. Icarus 248, 478-498 (2015). https://doi.org/10.1016/j.icarus.2014.10.047

R.M. Goody, Sources and sinks of climate entropy. Q. J. R. Meteorol. Soc. 126, 1953-1970 (2000)

R.M. Goody, R.A. West, L. Chen, D. Crisp, The correlated-k method for radiation calculations in nonhomogeneous atmospheres. J. Quant. Spectrosc. Radiat. Transf. 42, 539-550 (1989)

E. Goos, U. Riedel, L. Zhao, L. Blum, Phase diagrams of $\mathrm{CO}_{2}$ and $\mathrm{CO}_{2}-\mathrm{N}_{2}$ gas mixtures and their application in compression processes. Energy Proc. 4, 3778-3785 (2011). https://doi.org/10.1016/ j.egypro.2011.02.312

D. Grassi, P. Drossart, G. Piccioni, N.I. Ignatiev, L.V. Zasova, A. Adriani, M.L. Moriconi, P.G.J. Irwin, A. Negrao, A. Migliorini, Retrieval of air temperature profiles in the Venusian Mesosphere from VIRTIS-M data: description and validation of algorithms. J. Geophys. Res. (2008). https://doi.org/ 10.1029/2008JE003075

D. Grassi, A. Migliorini, L. Montabone, S. Lebonnois, A. Cardesìn-Moinelo, G. Piccioni, P. Drossart, L.V. Zasova, Thermal structure of Venusian night-time mesosphere as observed by VIRTIS-Venus Express. J. Geophys. Res., Planets 115, 9007 (2010). https://doi.org/10.1029/2009JE003553

D. Grassi, R. Politi, N.I. Ignatiev, C. Plainaki, S. Lebonnois, P. Wolkenberg, L. Montabone, A. Migliorini, G. Piccioni, P. Drossart, The Venus nighttime atmosphere as observed by the VIRTIS-M instrument. Average fields from the complete infrared data set. J. Geophys. Res., Planets 119, 837-849 (2014). https://doi.org/10.1002/2013JE004586

H. Grassl, The climate at maximum entropy production by meridional and atmospheric heat fluxes. Q. J. R. Meteorol. Soc. 107, 153-166 (1990)

B. Grieger, N.I. Ignatiev, N.M. Hoekzema, H.U. Keller, Indication of a near surface cloud layer on Venus from reanalysis of Venera 13/14 spectrophotometer data, in Proceedings of the International Workshop Planetary Probe Atmospheric Entry and Descent Trajectory Analysis and Science, ed. by A. Wilson Lisbon, Portugal, 6-9 October 2003 (ESA Publications Division, Noordwijk, 2004), pp. 63-70

J.E. Hansen, J.W. Hovenier, Interpretation of the polarization of Venus. J. Atmos. Sci. 31, 1137-1160 (1974)

R. Haus, Radiative energy balance of Venus: an approach to parameterize thermal cooling and solar heating rates. Icarus 284(1), 216-232 (2017). https://doi.org/10.1016/j.icarus.2016.11.025

R. Haus, H. Goering, Radiative energy balance in the Venus mesosphere. Icarus 84, 62-82 (1990)

R. Haus, D. Kappel, G. Arnold, Self-consistent retrieval of temperature profiles and cloud structure in the northern hemisphere of Venus using VIRTIS/VEX and PMV/VENERA-15 radiation measurements. Planet. Space Sci. 89, 77-101 (2013). https://doi.org/10.1016/j.pss.2013.09.020

R. Haus, D. Kappel, G. Arnold, Radiative energy balance of Venus: an approach to parameterize thermal cooling and solar heating rates. Icarus 284, 216-232 (2014). https://doi.org/10.1016/j.icarus.2016.11.025 
R. Haus, D. Kappel, S. Tellmann, G. Arnold, P. Drossart, B. Häusler, Radiative energy balance of Venus based on improved models of the middle and lower atmosphere. Icarus 272, 178-205 (2016). https://doi.org/10.1016/j.icarus.2016.02.048

B. Häusler et al., Radio Science investigations by VeRa onboard the Venus Express spacecraft. Planet. Space Sci. 54, 1315-1335 (2006)

B. Häusler et al., Venus atmospheric, ionospheric, surface and interplanetary radio-wave propagation studies with the VeRa radio-science experiment, in ESA Scientific Publication, vol. SP-1295 (2007), pp. 1-30. http://sci.esa.int/science-e/www/object/index.cfm?fobjectid=41535

A.E. Hedin, H.B. Niemann, W.T. Kasprzak, Global empirical model of the Venus thermosphere. J. Geophys. Res. 88, 73-83 (1983)

D. Hendry, A. Miller, N. Wilkinson, M. Wickramathilaka, R. Espanani, W. Jacoby, Exploration of high pressure equilibrium separations of nitrogen and carbon dioxide. J. CO2 Util. 3-4, 37-43 (2013). https://doi.org/10.1016/j.jcou.2013.09.002

M. Herrmann et al., A new method for the detection of small-scale wave-like features in the venusian atmosphere with the Venus Express radio science experiment VeRa, in European Planetary Science Congress, 2014. EPSC Abstracts, vol. 9 (2015)

J. Hilsenrath, C.W. Beckett, W.S. Benedict, L. Fano, H.J. Hoge, F. Musi, R.L. Nuttall, Y.S. Touloukian, Tables of Thermodynamic and Transport Properties of Air, Argon, Carbon Dioxide, Carbon Monoxide, Hydrogen, Nitrogen, Oxygen, and Steam (Pergamon, New York, 1960)

D.P. Hinson, J.M. Jenkins, Magellan radio occultation measurements of atmospheric waves on Venus. Icarus 114, 310-327 (1995)

H.T. Howard, G.L. Tyler, G. Fjeldbo, A.J. Kliore, G.S. Levy, D.L. Brunn, R. Dickinson, R.E. Edelson, W.L. Martin, R.B. Postal, B. Seidel, T.T. Sesplaukis, D.L. Shirley, C.T. Stelzried, D.N. Sweetnam, A.I. Zygielbaum, P.B. Esposito, J.D. Anderson, I.I. Shapiro, R.D. Reasenberg, Venus: Mass, gravity field, atmosphere, and ionosphere as measured by the Mariner 10 dual-frequency radio system. Science 183, 1297-1301 (1974). https://doi.org/10.1126/science.183.4131.1297

C.T. Hua, G. Courtes, N. Huu-Doan, The detection of anhydrous sulfur $\mathrm{SO}_{2}$ and, doubtless, $\mathrm{CS}_{2}$ in the atmosphere of Venus. Acad. Sci. Paris C. R., Ser. B, Sci. Phys. 288(11), 187-190 (1979a)

N.I. Ignatiev, V.I. Moroz, B.E. Moshkin, A.P. Ekonomov, V.I. Gnedykh, A.V. Grigoriev, I.V. Khatuntsev, Water vapor the lower atmosphere of Venus: a new analysis of optical spectra measured by entry probes. Planet. Space Sci. 45, 427-438 (1997)

N.I. Ignatiev, D.V. Titov, G. Piccioni, P. Drossart, W.J. Markiewicz, V. Cottini, Th. Roatsch et al., Altimetry of the Venus cloud tops from the Venus Express observations. J. Geophys. Res. 114, 405-414 (2009)

T. Imamura, H. Ando, S. Tellmann, M. Pätzold, B. Häusler, A. Yamazaki, T.M. Sato, K. Noguchi, Y. Futaana, J. Oschlisniok, S. Limaye, R.K. Choudhary, Y. Murata, H. Takeuchi, C. Hirose, T. Ichikawa, T. Toda, A. Tomiki, T. Abe, Z. Yamamoto, H. Noda, T. Iwata, S. Murakami, T. Satoh, T. Fukuhara, K. Ogohara, K. Sugiyama, H. Kashimura, S. Ohtsuki, S. Takagi, Y. Yamamoto, N. Hirata, G.L. Hashimoto, M. Yamada, M. Suzuki, N. i Ishii, T. Hayashiyama, Y.J. Lee, M. Nakamura, Initial performance of the radio occultation experiment in the Venus orbiter mission Akatsuki. Earth Planets Space 69, 117 (2017). https://doi.org/10.1186/s40623-017-0722-3

A.P. Ingersoll, The runaway greenhouse: a history of water on Venus. J. Atmos. Sci. 26, 1191-1198 (1969)

W.-H. Ip, J.A. Fernandez, Exchange of condensed matter among the outer and terrestrial proto-planets and the effect on surface impact and atmospheric accretion. Icarus 74, 47-61 (1988)

W.B. Irvine, Monochromatic phase curves and albedos for Venus. J. Atmos. Sci. 25, 610-616 (1968). https://doi.org/10.1175/1520-0469

M.N. Izakov, Dissipation of buoyancy waves and turbulence in the atmosphere of Venus. Sol. Syst. Res. 44(6), 475-486 (2010)

J.M. Jenkins, Radio occultation studies of Venus' atmosphere with Magellan. Final Technical Report on Grant NAGW-4450, NASA/GSFC, Greenbelt, MD (1998)

J.M. Jenkins, P.G. Steffes, D.P. Hinson, J.D. Twicken, G.L. Tyler, Radio occultation studies of the Venus atmosphere with the Magellan Spacecraft, 2: results from the October 1991 experiments. Icarus 110(1), 79-94 (1994). https://doi.org/10.1006/icar.1994.1108

D. Kappel, G. Arnold, R. Haus, G. Piccioni, P. Drossart, Refinements in the data analysis of VIRTISM-IR Venus nightside spectra. Adv. Space Res. 50(2), 228-255 (2012). https://doi.org/10.1016/ j.asr.2012.03.029

W.T. Kasprzak, H.B. Niemann, A.E. Hedin, Neutral composition measurements by the Pioneer Venus Neutral Mass Spectrometer during orbiter re-entry. J. Geophys. Res. 20, 2747-2750 (1993)

W.T. Kasprzak, G.M. Keating, N.C. Hsu, A.I.F. Stewart, W.B. Colwell, S.W. Bougher, Solar activity behavior of the ionosphere, in Venus II, ed. by S.W. Bougher, D.M. Hunten, R.J. Philips (The University of Arizona Press, Tucson, 1997), pp. 225-257 
J.F. Kasting, Runaway and moist greenhouse atmospheres and the evolution of Earth and Venus. Icarus 74, 472-494 (1988)

J.F. Kasting, J.B. Pollack, T.P. Ackerman, Response of Earth's atmosphere to increases in solar flux and implications for loss of water from Venus. Icarus 57, 335-355 (1984)

G. Keating, J.Y. Nicholson, L.R. Lake, Venus upper atmosphere structure. J. Geophys. Res. 85, 7941-7956 (1980)

V.V. Kerzhanovich, M.Ya. Marov, The atmospheric dynamics of Venus according to Doppler measurements by the Venera entry probes, in Venus, ed. by D.M. Hunten, L. Colin, T.M. Donahue, V.I. Moroz (The University of Arizona Press, Tucson, 1983), pp. 766-778

I.V. Khatuntsev, M.V. Patsaeva, D.V. Titov, N.I. Ignatiev, A.V. Turin, S.S. Limaye, W.J. Markiewicz, M. Almeida, Th. Roatsch, R. Moissl, Cloud level winds from the Venus Express Monitoring Camera imaging. Icarus 226(1), 140-158 (2014). https://doi.org/10.1016/j.icarus.2013.05.018

I.V. Khatuntsev, M.V. Patsaeva, D.V. Titov, N.I. Ignatiev, A.V. Turin, A.A. Fedorova, W.J. Markiewicz, Winds in the middle cloud deck from the near-IR imaging by the Venus Monitoring Camera onboard Venus Express. J. Geophys. Res., Planets 122, 2312-2327 (2017). https://doi.org/10.1002/2017JE005355

A.J. Kliore, Recent results on the Venus atmosphere from pioneer Venus radio occultations. Adv. Space Res. 5(9), 41-49 (1985). https://doi.org/10.1016/0273-1177(85)90269-8

A.J. Kliore, I.R. Patel, Vertical structure of the atmosphere of Venus from Pioneer Venus orbiter radio occultations. J. Geophys. Res. 85, 7957-7962 (1980)

A. Kliore, I.R. Patel, Thermal structure of the atmosphere of Venus from Pioneer Venus radio occultations. Icarus 52, 320-334 (1982)

A. Kliore, D.L. Cain, G.S. Levy, R. Von Eshleman, G. r Fjeldbo, F.D. Drake, Occultation experiment: results of the first direct measurement of Mars's atmosphere and ionosphere. Science 149, 1243-1248 (1965). https://doi.org/10.1126/science.149.3689.1243

A. Kliore, G.S. Levy, D.L. Cain, G. Fjeldbo, S.I. Rasool, Atmosphere and ionosphere of Venus from the Mariner V S-band occultation measurement. Science 158, 1683-1688 (1967)

A. Kliore et al., Venus international reference atmosphere. Adv. Space Res. 5(11), 307 (1985)

R.G. Knollenberg, D.M. Hunten, The microphysics of the clouds of Venus: results of the Pioneer Venus particle size spectrometer experiment. J. Geophys. Res. 85, 8039-8058 (1980)

M.A. Kolosov, O.I. Yakovlev, S.S. Matyugov, T.S. Timofeeva, E.V. Chub, G.D. Yakovleva, I.E. Kalashnikov, The daytime atmosphere of Venus according to results of radio occultation by means of Venera 9 and 10 satellites. Kosm. Issled. 16, 278-284 (1978)

V.A. Krasnopolsky, Photochemistry of the Atmospheres of Mars and Venus, in Physics and Chemistry in Space, vol. 13, ed. by U. von Zahn (Springer, New York, 1986)

V.A. Krasnopolsky, On the iron chloride aerosol in the clouds of Venus. Icarus 286, 134-137 (2017)

G.P. Kuiper, Identification of the Venus cloud layers. Commun. Lunar Planet. Lab. 6, 229-245 (1969)

M. Kumar, J.S. Francisco, Elemental sulfur aerosol-formign mechanism. Proc. Natl. Acad. Sci. (2014). https://doi.org/10.1007/pnas.1620870114

O. Kunz, W. Wagner, The GERG-2008 wide-range equation of state for natural gases and other mixtures: an expansion of gerg-2004. J. Chem. Eng. Data 57(11), 3032-3091 (2012)

A.D. Kuzmin, Radio astronomical studies of Venus, in Venus, ed. by D. Hunten, L. Colin, V.I. Moroz (University of Arizona Press, Tucson, 1983)

P. Laven, How are glories formed? Appl. Opt. 44(27), 5675-5683 (2005)

S. Lebonnois, G. Schubert, The deep atmosphere of Venus and the possible role of density-driven separation of $\mathrm{CO}_{2}$ and $\mathrm{N}_{2}$. Nat. Geosci. 10(7), 473-477 (2017). https://doi.org/10.1038/ngeo2971

S. Lebonnois, C. Lee, M. Yamamoto, J. Dawson, S.R. Lewis, J. Mendonca, P. Read, H.F. Parish, G. Schubert, L. Bengtsson, D. Grinspoon, S.S. Limaye, H. Schmidt, H. Svedhem, D.V. Titov, Models of Venus atmosphere, in Towards Understanding the Climate of Venus, ed. by Bengtsson et al. (Springer, New York, 2013), pp. 129-156

Y.J. Lee, Venus cloud structure and radiative energy balance of the mesosphere. $\mathrm{PhD}$ thesis, TU Braunschweig (2011)

Y.J. Lee, D.V. Ditov, S. Tellmann, A. Piccialli, N. Ignatiev, M. Pätzold, B. Häusler, G. Piccioni, P. Drossart, Vertical structure of the Venus cloud top from the VeRa and VIRTIS observations onboard Venus Express. Icarus 217, 599-609 (2012). https://doi.org/10.1016/j.icarus.2011.07.001

Y.J. Lee, T. Imamura, S.E. Schröder, E. Marcq, Long-term variations of the UV contrast on Venus observed by the Venus Monitoring Camera on board Venus Express. Icarus 253, 1-15 (2015). doi.org/10.1016/j.icarus.2015.02.015

E. Lellouch, T. Clancy, D. Crisp, A.J. Kliore, D. Titov, S.W. Bougher, Monitoring of mesospheric structure and dynamics, in Venus-II, ed. by S.W. Bougher, D.M. Hunten, R.J. Phillips (The University of Arizona Press, Tucson, 1997), pp. 295-324 
C.B. Leovy, Rotation of the upper atmosphere of Venus. J. Atmos. Sci. 30, 1218-1220 (1973). https://doi.org/ 10.1175/1520-0469(1973)030<1218:ROTUAO >2.0.CO;2

C.B. Leovy, Control of the homopause level. Icarus 50(2-3), 311-321 (1982). https://doi.org/10.1016/ 0019-1035(82)90128-2

S.R. Lewis, J. Dawson, S. Lebonnois, M. Yamamoto, Modeling efforts, in Towards Understanding the Climate of Venus, ed. by Bengtsson et al. (Springer, New York, 2013), pp. 111-128

S.S. Limaye, Morphology and movements of polarization features on Venus. Icarus 57, 362-385 (1984)

S.S. Limaye, Venus atmospheric circulation: observations and implications of the thermal structure. Adv. Space Res. 5(9), 51-62 (1985)

S.S. Limaye, Observed Cloud level circulation on Venus: temporal variations and solar longitude dependence. Middle atmosphere of Venus, in Veroffentlichungen des Forschungsbereichs Geo- und Kosmoswissenschaften, vol. 18, ed. by K. Schafer, D. Spankuch (Akademie-Verlag, Berlin, 1990), pp. 121-140

S.S. Limaye, V.E. Suomi, Cloud motions on Venus - global structure and organization. J. Atmos. Sci. 38, $1220-1235(1981)$

S.S. Limaye, J.P. Kossin, C. Rozoff, G. Piccioni, D.V. Titov, W.J. Markiewicz, Vortex circulation on Venus: dynamical similarities with terrestrial hurricanes. Geophys. Res. Lett. 36(4), L04204 (2009). https://doi.org/10.1029/2008GL036093

S.S. Limaye, S. Lebonnois, A. Mahieux, M. Pätzold, S. Bougher, S. Bruinsma, S. Chamberlain, R.T. Clancy, J.-C. Gérard, G. Gilli, D. Grassi, R. Haus, M. Herrmann, T. Imamura, E. Kohler, P. Krause, A. Migliorini, F. Montmessin, C. Pere, M. Persson, A. Piccialli, M. Rengel, A. Rodin, B. Sandor, M. Sornig, H. Svedhem, S. Tellmann, P. Tanga, A.C. Vandaele, T. Widemann, C.F. Wilson, I. Müller-Wodarg, L. Zasova, The thermal structure of the venus atmosphere: intercomparison of Venus Express and ground based observations of vertical temperature and density profiles. Icarus 294, 124-155 (2017). https://doi.org/10.1016/j.icarus.2017.04.020

S.S. Limaye, S. Watanabe, A. Yamazaki, M. Yamada, T. Satoh, T.M. Sato, M. Nakamura, M. Taguchi, T. Fukuhara, T. Imamura, T. Kouyama, Y.-J. Lee, T. Horinouchi, J. Peralta, N. Iwagami, G.L. Hashimoto, S. Takagi, S. Ohtsuki, S. Murakami, Y. Yamamoto, K. Ogohara, H. Ando, K. Sugiyama, N. Ishii, T. Abe, C. Hirose, M. Suzuki, N. Hirata, E.F. Young, A.C. Ocampo, Venus looks different from day to night across wavelengths: morphology from Akatsuki multispectral images. Earth Planets Space 70, 24 (2018a). https://doi.org/10.1186/s40623-018-0789-5

S.S. Limaye, R. Rakesh, D.J. Smith David, A.H. Ansari, G.P. Słowik, P. Vaishampayan, Venus' spectral signatures and the potential for life in the clouds. Astrobiology (2018b). https://doi.org/10.1089/ ast.2017.1783

V.M. Linkin, J.E. Blamont, A.N. Lipatov, S.I. Devyatkin, A.V. Dyachkov, S.P. Ignatova, V.V. Kerzhanovich, L.I. Khlyustova, C. Malique, Y.V. Sanotskii, A.A. Shurupov, B.I. Stadnyk, P.G. Stolyarchuk, A.V. Terterashvili, Vertical thermal structure in the Venus atmosphere from Provisional VEGA-2 temperature and pressure data. Sov. Astron. Lett. 12, 100-105 (1986a)

V.M. Linkin, V.V. Kerzhanovich, A.N. Lipatov, A.A. Shurupov, A. Sieff, B. Ragent, R.E. Young, A.P. Ingersoll, D. Crisp, L.S. Elson, R.A. Preston, J.E. Blamont, Thermal structure of the Venus atmosphere in the middle cloud layer. Science 231, 1420-1422 (1986b)

K.N. Liou, Radiation and Cloud Processes in the Atmosphere (Oxford University Press, New York, 1992)

R.D. Lorenz, J.I. Lunine, P.G. Withers, C.P. McCay, Titan, Mars, and Earth: Entropy production by latitudinal heat transport. Geophys. Res. Lett. 28, 415-418 (2001)

V. Lucarini, K. Fraedrich, F. Lunkeit, Thermodynamic analysis of snowball Earth hysteresis experiment: efficiency, entropy production and irreversibility. Q. J. R. Meteorol. Soc. 136, 2-11 (2010)

J.R. Lyons, An estmate of the equilibrium speciaton of sulfur vapor over solid sulfur and implicatons for planetary atmospheres. J. Sulfur Chem. 29(3-4), 269-279 (2008). https://doi.org/10.1080/ 17415990802195615

B. Lyot, Recherrches sur la polarization de la lumiere des planets et de qulques substances terrestres. Ann. Observ. Paris (Meudon) 8, 161 (1929)

A. Mahieux, S. Berkenbosch, R. Clairquin, D. Fussen, N. Mateshvili, E. Neefs, D. Nevejans, B. Ristic, A.C. Vandaele, V. Wilquet, D. Belyaev, A. Fedorova, O. Korablev, E. Villard, F. Montmessin, J.L. Bertaux, In-flight performance and calibration of SPICAV/SOIR on-board Venus Express. Appl. Opt. 47, 22522265 (2008)

A. Mahieux, A.C. Vandaele, E. Neefs, S. Robert, V. Wilquet, R. Drummond, A. Federova, J.-L. Bertaux, Densities and temperatures in the Venus mesosphere and lower thermosphere retrieved from SOIR on board Venus Express: retrieval technique. J. Geophys. Res. 115(E12), E12014 (2010). https://doi.org/10.1029/2010JE003589

A. Mahieux, A.C. Vandaele, S. Robert, V. Wilquet, R.F. Montmessin, J.-L. Bertaux, Densities and temperatures in the Venus mesosphere and lower thermosphere retrieved from SOIR on board Venus Express: carbon dioxide measurements at the Venus terminator. J. Geophys. Res. 117(E7), E07001 (2012). https://doi.org/10.1029/2012JE004058 
A. Mahieux, A.C. Vandaele, S.W. Bougher, R. Drummond, S. Robert, V. Wilquet, S. Chamberlain, A. Piccialli, F. Montmessin, S. Tellmann, M. Pätzold, B. Häusler, J.L. Bertaux, Update of the Venus density and temperature profiles at high altitude measured by SOIR on board Venus Express. Planet. Space Sci. 113-114, 309-320 (2015a). https://doi.org/10.1016/j.pss.2015.02.002

A. Mahieux, A.C. Vandaele, S. Robert, V. Wilquet, R. Drummond, M.A. López Valverde, Rotational temperatures of Venus upper atmosphere as measured by SOIR on board Venus Express. Planet. Space Sci. (2015b). https://doi.org/10.1016/j.pss.2014.12.020

A. Mahieux, A.C. Vandaele, V. Wilquet, J.T. Erwin, R.V. Yelle, A 1-D non LTE radiative transfer model of the Venus mesosphere and thermosphere: model description and comparison with SOIR/VEx. Geophys. Res. Abstr. 17, EGU2015-9030 (2016)

A. Mallama, Characterization of terrestrial exoplanets based on the phase curves and albedos of Mercury, Venus and Mars. Icarus 204, 11-14 (2009)

A.D. Mallama, D. Wang, R.A. Howard, Venus phase function and forward scattering from $\mathrm{H}_{2} \mathrm{SO}_{4}$. Icarus 182, 10-22 (2006). https://doi.org/10.1016/j.icarus.2005.12.014

E. Marcq, J.L. Bertaux, F. Montmessin, D. Belyaev, Variations of sulphur dioxide at the cloud top of Venus's dynamic atmosphere. Nat. Geosci. 6, 25-28 (2013)

E. Marcq, F.P. Mills, C.D. Parkinson, A.C. Vandaele, Composition and chemistry of the neutral atmosphere of Venus. Space Sci. Rev. (2018). https://doi.org/10.1007/s11214-017-0438-5

W.J. Markiewicz, D.V. Titov, S.S. Limaye, H.U. Keller, N.I. Ignatiev, R. Jaumann, N. Thomas et al., Morphology and dynamics of the upper cloud layer of Venus. Nature 450, 633-636 (2007a). https://doi.org/ 10.1038 /nature06320

W.J. Markiewicz et al., Venus monitoring camera for Venus Express. Planet. Space Sci. 55, 1701-1711 (2007b)

W.J. Markiewicz, E. Petrova, O. Shalygina, M. Almeida, D.V. Titov, S.S. Limaye, N. Ignatiev, T. Roatsch, K.D. Matz, Glory on Venus cloud tops and the unknown UV absorber. Icarus 234, 200-203 (2014). https://doi.org/10.1016/j.icarus.2014.01.030

M.Ya.Marov, V.S. Avduevsky, V.V. Kerzhanovich, M.K. Rozhdestvensky, N.F. Borodin, O.L. Ryabov, Venera 8: measurements of temeprature, pressure, andwind velocity on the illuminated side of Venus. J. Atmos. Sci. 30, 1210-1214 (1973)

C.H. Mayer, T.P. Mcullough, R.M. Slonaker, Observations of Venus at $3.14 \mathrm{~cm}$ wavelength. Astrophys. J. 127, 1-10 (1958)

W.B. McKinnon, K.J. Zahnle, B.A. Ivanov, H.J. Melosh, Cratering on Venus: models and observations, in Venus II, ed. by S.W. Bougher, D.M. Hunten, R.J. Phillips (University of Arizona Press, Tucson, 1997), pp. 969-1014

V.S. Meadows, D. Crisp, Ground-based near-infrared observations of the Venus night side: the thermal structure and water abundance near the surface. J. Geophys. Res. 101, 4595-4622 (1996)

A.D. Migliorini, D. Grassi, L. Montabone, S. Lebonnois, P. Drossart, G. Piccioni, Investigation of air temperature on the nightside of Venus derived from VIRTIS-H on board Venus-Express. Icarus 217(2), 640-647 (2012). https://doi.org/10.1016/j.icarus.2011.07.013

N. Mohan, S. Roy, G. Swarup, D. Oberoi, N. Mohan Ramanujam, S. Raju, A. Bhardwaj, Radio observation of Venus at meter wavelengths using the GMRT. Icarus 297(Suppl. C), 119-125 (2017). https://doi.org/10.1016/j.icarus.2017.06.026

D. Mondelain, S. Vasilchenko, P. Čermák, S. Kassi, A. Campargue, The CO2 absorption spectrum in the 2.3 mm transparency window by high sensitivity CRDS: (II) self-absorption continuum. J. Quant. Spectrosc. Radiat. Transf. 187, 38-43 (2017)

F. Montmessin, J.-L. Bertaux, F. Lefevre, E. Marcq, D. Belyaev, J.-C. Gerard, O. Korablev, A. Fedorova, V. Sarago, A.C. Vandaele, A layer of ozone detected in the nightside upper atmosphere of Venus. Icarus 216(1), 82-85 (2011). https://doi.org/10.1016/j.icarus.2011.08.010

V.I. Moroz, Stellar magnitude and albedo data of Venus, in Venus, ed. by D.M. Hunten, L. Colin, T.M. Donahue, V.I. Moroz (The University of Arizona Press, Tucson, 1983), pp. 27-35

V.I. Moroz, A.P. Ekonomov, Yu.M. Golovin, B.E. Moshkin, N.F. San'ko, Solar radiation scattered in the Venus atmosphere: the Venera 11, 12 data. Icarus 53, 509-537 (1983)

V.I. Moroz, A.P. Ekonomov, B.E. Moshkin, H.E. Revercomb, L.A. Sromovsky, J.T. Schofield, D. Spaenkuch, F.W. Taylor, M.G. Tomasko, Solar and thermal radiation in the Venus atmosphere. Adv. Space Res. 5, 197-232 (1985)

N.I. Moskalenko, Y.A. Il'in, S.N. Parzhin, L.V. Rodinovon, Pressure-induced IR radiation absorption in atmospheres. Bull. Acad. Sci. USSR, Atmos. Ocean. Phys. 15, 632-637 (1979)

N.T. Mueller, C. Tsang, S. Lebonnois, S. Smrekar, Regional Venus surface temperature variations in models and infrared observations, in 49th Lunar and Planetary Science Conference (2018)

I.C.F. Müller-Wodarg, J.M. Forbes, G.M. Keating, The thermosphere of Venus and its exploration by a Venus Express accelerometer experiment. Planet. Space Sci. 54, 1415-1424 (2006) 
M. Nakamura, T. Imamura, M. Ueno, N. Iwagami, T. Satoh, S. Watanabe, M. Taguchi, Y. Takahashi, M. Suzuki, T. Abe, G.L. Hashimoto, T. Sakanoi, S. Okano, Y. Kasaba, J. Yoshida, M. Yamada, N. Ishii, T. Yamada, K-I. Oyama PLANET-C, Venus Climate Orbiter mission of Japan. Planet. Space Sci. 55, 1831-1842 (2007)

M. Nakamura, Y. Kawakatsu, C. Hirose, T. Imamura, N. i Ishii, T. Abe, A. Yamazaki, M. Yamadab, K. Ogohara, K. Uemizu, T. Fukuhara, S. Ohtsuki, T. Satoh, M. Suzuki, M. Ueno, J. Nakatsuka, N. Iwagami, M. Taguch, S. Watanabe, Y. Takahashi, G.L. Hashimoto, H. Yamamoto, Return to Venus of the japanese Venus Climate Orbiter AKATSUKI. Acta Astronaut. 93, 384-389 (2014). https://doi.org/10.1016/j.actaastro.2013.07.027

M. Nakamura, T. Imamura, N. Ishii, T. Abe, Y. Kawakatsu, C. Hirose, T. Satoh, M. Suzuki, M. Ueno, A. Yamazaki, N. Iwagami, S. Watanabe, M. Taguchi, T. Fukuhara, Y. Takahashi, M. Yamada, M. Imai, S. Ohtsuki, K. Uemizu, G.L. Hashimoto, M. Takagi, Y. Matsuda, K. Ogohara, N. Sato, Y. Kasaba, T. Kouyama, N. Hirata, R. Nakamura, Y. Yamamoto, T. Horinouchi, M. Yamamoto, Y-Y. Hayashi, H. Kashimura, K. Sugiyama, T. Sakanoi, H. Ando, S. Murakami, T.M. Sato, S. Takagi, K. Nakajima, J. Peralta, Y.J. Lee, J. Nakatsuka, T. Ichikawa, K. Inoue, T. Toda, H. Toyota, S. Tachikawa, S. Narita, T. Hayashiyama, A. Hasegawa, Y. Kamata, AKATSUKI returns to Venus. Earth Planets Space 68, 75 (2016). https://doi.org/10.1186/s40623-016-0457-6

D. Nevejans, E. Neefs, A.C. Vandaele, C. Müller, D. Fussen, S. Berkenbosch, R. Clairquin, O. Korablev, A. Fedorova, J.L. Bertaux, First operations of the SOIR occultations infrared spectrometer in Venus orbit, in European Planetary Science Congress, Berlin, Germany, 18-22 Sept. (2006)

M. Newman, G. Schubert, A.J. Kliore, I.R. Patel, Zonal winds in the middle atmosphere of Venus from Pioneer Venus radio occultation data. J. Atmos. Sci. 41, 1901-1913 (1984)

H.B. Niemann, W.T. Kasprzak, A.E. Hedin, D.M. hunten, N.W. Spencer, Mass spectrometric measurements of the neutral gas composition of the thermosphere and exosphere of Venus. J. Geophys. Res. 85, 78177827 (1980)

D. Oertel, V.I. Moroz, D. Spaenkuch, V.M. Linkin, H. Jahn, V.V. Kerzhanovich, H. Becker-Ross, I.A. Matsygorin, K. Stadthaus, A.N. Lipatov, J. Nopirakowsky, A.A. Shurupov, W. Dohler, L.V. Zasova, K. Schaefer, E.A. Ustinov, J. Guldner, R. Dubois, Infrared spectrometry from Venera-15 and Venera-16. Adv. Space Res. 5, 25 (1987)

D.M.H. Ossa, R.R. Oliveira, M.T. Murakami, R. Vicentini, A.J. Costa-Filho, F. Alexandrino, L.M.M. Ottobono, O. Garcia, Expression, purification and spectroscopic analysis of an HdrC: an iron-sulfur clustercontaining protein from Acidithiobacillus ferrooxidans. Process Biochem. 46(6), 1335-1341 (2011). https://doi.org/10.1016/j.procbio.2011.03.001

V.I. Oyama, G.C. Carle, F. Woeller, J.B. Pollack, R.T. Reynolds, R.A. Craig, Pioneer Venus gas chromatography of the lower atmosphere of Venus. J. Geophys. Res. 85(A13), 7891-8902 (1980)

G.W. Paltridge, Global dynamics and climate - a system of minimum entropy exchange. Q. J. R. Meteorol. Soc. 101, 475-484 (1975)

P.N. Peplowski, D.J. Lawrence, Nitrogen Content of Venus' Upper Atmosphere from the MESSENGER Neutron Spectrometer, in 47th Lunar and Planetary Science Conference (2016), Abstract 1177. https:// www.hou.usra.edu/meetings/lpsc2016/pdf/1177.pdf

J. Peralta, M.A. López-Valverde, G. Gilli, A. Piccialli, Dayside temperatures in the Venus upper atmosphere from Venus Express/VIRTIS nadir measurements at $4.3 \mu \mathrm{m}$. Astron. Astrophys. 585, A53 (2016). https://doi.org/10.1051/0004-6361/201527191

S. Pérez-Hoyos, A. Sánchez-Lavega, A. García-Muñoz, P.G.J. Irwin, J. Peralta, G. Holsclaw, W.M. McClintock, J.F. Sanz-Requena, Venus upper clouds and the UV absorber from MESSENGER/MASCS observations. J. Geophys. Res., Planets 123, 145-162 (2017). https://doi.org/10.1002/2017JE005406

M. Persson, Venus thermosphere densities as revealed by Venus Express torque and accelerometer data. Master's thesis, http://pure.ltu.se/portal/files/104159817/LTU-EX-2015-104154994.pdf (2015)

E. Petrova, Glory on Venus and selection among the unknown UV absorbers. Icarus 306, 163-170 (2018)

E.V. Petrova, O.S. Shalygina, W.J. Markiewicz, The VMC/VEx photometry at small phase angles: glory and the physical properties of particles in the upper cloud layer of Venus. Planet. Space Sci. 113-114, 120-134 (2015). https://doi.org/10.1016/j.pss.2014.11.013

E. Pettit, S.B. Nicholson, Temperatures on the bright side and dark side of Venus. Publ. Astron. Soc. Pac. 67, 293-303 (1955)

A. Piccialli, D.V. Titov, D. Grassi, I. Khatuntsev, P. Drossart, G. Piccioni, A. Migliorini, Cyclostrophic winds from the Visible and Infrared Thermal Imaging Spectrometer temperature sounding: a preliminary analysis. J. Geophys. Res., Planets 113, 147-155 (2008)

A. Piccialli, S. Tellmann, D.V. Titov, S.S. Limaye, I.V. Khatuntsev, M. Pätzold, B. Häusler, Dynamical properties of the Venus mesosphere from the radio-occultation experiment VeRa onboard Venus Express. Icarus 217, 669-681 (2012). https://doi.org/10.1016/j.icarus.2011.07.016 
A. Piccialli, D.V. Titov, A. Sanchez-Lavega, J. Peralta, O. Shalygina, W.J. Markiewicz, H. Svedhem, High latitude gravity waves at the Venus cloud tops as observed by the Venus Monitoring Camera on board Venus Express. Icarus 227, 94-111 (2014)

A. Piccialli, F. Montmessin, D. Belyaev, A. Mahieux, A. Fedorova, E. Marcq, J.-L. Bertaux, S. Tellmann, A.C. Vandaele, O. Korablev, Thermal structure of Venus nightside upper atmosphere measured by stellar occultations with SPICAV/Venus Express. Planet. Space Sci. (2015). https://doi.org/10.1016/j.pss.2014.12.009

A. Piccialli, R. Moreno, T. Encrenaz, T. Fouchet, E. Lellouch, T. Widemann, Mapping the thermal structure and minor species of Venus mesosphere with ALMA submillimeter observations. Astron. Astrophys. 606, A53 (2017). https://doi.org/10.1051/0004-6361/201730923

G. Piccioni, et al., VIRTIS: the Visible and Infrared Thermal Imaging Spectrometer, in ESA-SP-1295, Noordwjik, The Netherlands (2007a). http://sci.esa.int/venus-express/41537-virtis-the-visible-and-infraredthermal-imaging-spectrometer/\#

G. Piccioni, P. Drossart, A. Sanchez-Lavega, R. Hueso, F.W. Taylor, C. Wilson et al., South-polar features on Venus similar to those near the north pole. Nature 450, 637-640 (2007b)

J.B. Pollack, O.B. Toon, R. Boese, Greenhouse models of Venus' high surface temperature, as constrained by Pioneer Venus measurements. J. Geophys. Res. 85, 8223-8231 (1980a)

J.B. Pollack, O.B. Toon, R.C. Whitten, R. Boese, B. Ragent, M.G. Tomasko et al., Distribution and source of the UV absorption in Venus' atmosphere. J. Geophys. Res. 85, 8141-8150 (1980b)

J.B. Pollack, J.B. Dalton, D. Grinspoon, R.B. Wattson, R. Freedman, D. Crisp, D.A. Allen, B. Bezard, C. DeBergh, L.P. Giver, Q. Ma, R. Tipping, Near-infrared light from Venus' nightside: a spectroscopic analysis. Icarus 103, 1-42 (1993). https://doi.org/10.1006/icar.1993.1055

I. Prigogine, From Being to Becoming: Time and Complexity in the Physical Sciences (Freeman, New York, 1980)

S.I. Rasool, C. de Bergh, The runaway greenhouse and the accumulation of $\mathrm{CO}_{2}$ in the Venus atmosphere. Nature 226, 1037-1039 (1970)

P.L. Read, J. Barstow, B. Charnay, S. Chelvaniththilan, P.G.J. Irwin, S. Knight, S. Lebonnois, S.R. Lewis, J. Mendon, L. Montabone, Global energy budgets and 'Trenberth diagrams' for the climates of terrestrial and gas giant planets. Q. J. R. Meteorol. Soc. 142, 703-720 (2016). https://doi.org/10.1002/qj.2704

N.O. Renno, Multiple-equilibria in radiative-convective atmospheres. Tellus 49A, 423-438 (1997)

N.O. Renno, Comments on "Frictional dissipation in a precipitating atmosphere". J. Atmos. Sci. 58, 11731177 (2001)

N.O. Renno, A.P. Ingersoll, Natural convection as a heat engine: a theory for CAPE. J. Atmos. Sci. 53, 572-585 (1996)

H.E. Revercomb, L.A. Sromovsky, V.E. Suomi, R.W. Boese, Net thermal radiation in the atmosphere of Venus. Icarus 61, 521-538 (1985)

M. Roos-Serote, P. Drossart, Th. Encrenaz, E. Lellouch, R.W. Carlson, K.H. Baines, F.W. Taylor, S.B. Calcutt, The thermal structure and dynamics of the atmosphere of Venus between 70 and $90 \mathrm{~km}$ from the GalileoNIMS spectra. Icarus 114, 300-309 (1995)

P. Rosenblatt, S.L. Bruinsma, I.C. Müller-Wodarg, B. Häusler, H. Svedhem, J.C. Marty, First ever in situ observations of Venus' polar upper atmosphere density using the tracking data of the Venus Express Atmospheric Drag Experiment (VExADE). Icarus 217, 831-838 (2012)

W.B. Rossow, A.T. Del Genio, S.S. Limaye, L.D. Travis, P. Stone, Cloud morphology and motions from Pioneer Venus images. J. Geophys. Res. 85, 8107-8128 (1980)

L.S. Rothman, C.P. Rinsland, A. Goldman, S.T. Massie, D.P. Edwards, J.M. Flaud, A. Perrin, C. Camy-Peyret, V. Dana, J.Y. Mandin, J. Schroeder, A. McCann, R.R. Gamache, R.B. Watson, K. Yoshino, K.V. Chance, K.W. Jucks, L.R. Brown, V. Nemtchinov, R. Varanasi, The HITRAN molecular spectroscopic database and HAWKS (HITRAN Atmospheric Workstation): 1996 edition. J. Quant. Spectrosc. Radiat. Transf. 60, 665-710 (1998)

D.H. Rothman, D. Jacquemart, A. Barbe, D.C. Benner, M. Birk, L.R. Brown, M.R. Carleer, C. Chackerian, K.V. Chance, L.H. Coudert, V. Dana, V.M. Devi, J.M. Flaud, R.R. Gamache, A. Goldman, J.-M. Hartmann, K.W. Jucks, A.G. Maki, J.Y. Mandin, S.T. Massie, J. Orphal, A. Perrin, C.P. Rinsland, M.A.H. Smith, J. Tennyson, R.N. Tolchenov, R.A. Toth, J.V. Auwera, P. Varanasi, G. Wagner, The HITRAN 2004 molecular spectroscopic database. J. Quant. Spectrosc. Radiat. Transf. 96, 139-204 (2005)

C. Sagan, The radiation balance of Venus. Technical Report No. 32-34, Jet Propulsion Laboratory, NASA Contract No. NASW-6 (1960)

R.Z. Sagdeev, V.M. Linkin, V.V. Kerzhanovich, A.N. Lipatov, A.A. Shurupov, J.E. Blamont, D. Crisp, A.P. Ingersoll, L.S. Elson, R.A. Preston, C.E. Hildebrand, B. Ragent, A. Seiff, R.E. Young, G. Petit, L. Boloh, Y.N. Alexandrov, N.A. Armand, R.V. Bakitko, A.S. Selivanov, Overview of VeGa Venus balloon meteorological measurements. Science 231, 1411-1414 (1986). https://doi.org/10.1126/science.231.4744.1411 
A. Sánchez-Lavega, S. Lebonnois, T. Imamura, P. Read, D. Luz, The atmospheric dynamics of Venus. Space Sci. Rev. 212, 1541-1616 (2017)

B.J. Sandor, R.T. Clancy, First measurements of $\mathrm{ClO}$ in the Venus atmosphere - altitude dependence and temporal variation. Icarus 313, 15-24 (2018). https://doi.org/10.1016/j.icarus.2018.04.022

T.M. Sato, H. Sagawa, T. Kouyama, K. Mitsuyama, T. Satoh, S. Ohtsuki, M. Ueno, Y. Kasaba, M. Nakamura, T. Imamura, Cloud top structure of Venus revealed by Subaru/COMICS mid-infrared images. Icarus 243, 386-399 (2014). https://doi.org/10.1016/j.icarus.2014.09.004

K. Schäfer, V.M. Linkin, K. Dethloff, D.V. Pcaev, R. Dubois, V.V. Kerzhanovich, D. Spänkuch, L.V. Zasova, D. Oertel, Temperature and thermal wind fielf of the middle atmosphere of Venus from Venera 15 data, in Akademie der Wissenschaften der DDR, Heft 18, Middle Atmosphere of Venus, ed. by K. Schäfer, D. Spänkuch (Akademie-Verlag, Berlin, 1990), pp. 141-154

J.T. Schofield, F.W. Taylor, Net global thermal emission from the Venusian upper atmosphere. Icarus 52, 245-262 (1982)

J.T. Schofield, F.W. Taylor, Measurements of the mean, solar-fixed temperature and cloud structure of the middle atmosphere of Venus. Q. J. R. Meteorol. Soc. 109, 57-80 (1983)

G. Schubert, J.L. Mitchell, Planetary atmospheres as heat engines, in Comparative Climatology of Terrestrial Planets, ed. by S. Mackwell et al.(University of Arizona Press, Tucson, 2013), pp. 181-191

A. Seiff, Dynamical implications of the observed thermal contrasts in Venus' upper atmosphere. Icarus 51, 574-592 (1982)

A. Seiff, D.B. Kirk, Structure of the Venus mesosphere and lower thermospere from measurements during entry of the Pioneer Venus probes. Icarus 49, 49-70 (1982)

A. Seiff, D.B. Kirk, R.E. Young, R.C. Blanchard, J.T. Findlay, G.M. Kelly, S.C. Sommer, Measurements of thermal structure and thermal contrasts in the atmosphere of Venus and related dynamical observations: Results from the four Pioneer Venus probes. J. Geophys. Res. 85, 7903-7933 (1980)

A. Seiff, Thermal structure of the atmosphere of Venus, in Venus, ed. by D.M. Hunten et al.(University of Arizona Press, Tuscon, 1983), pp. 215-279

A. Seiff, J.T. Schofield, A.J. Kliore, F.W. Taylor, S.S. Limaye, H.E. Revercomb, L.A. Sromovsky, V.V. Kerzhanovich, V.I. Morozm, M.Ya. Marov, Models of the structure of atmosphere of Venus from surface to $100 \mathrm{~km}$ altitude. Adv. Space Res. 5, 3-57 (1985)

O.S. Shalygina, E.V. Petrova, W.J. Markiewicz, N. Ignatiev, E.V. Shalygin, Optical properties of the Venus upper clouds from the data obtained by Venus Monitoring Camera on-board the Venus Express. Planet. Space Sci. 113, 135-158 (2015). https://doi.org/10.1016/j.pss.2014.11.012

M. Snels, S. Stefani, G. Piccioni, B. Bèzard, Carbon dioxide absorption at high densities in the $1.18 \mu \mathrm{m}$ nightside transparency window of Venus. J. Quant. Spectrosc. Radiat. Transf. 133, 464-471 (2014)

G. Sonnabend, M. Sornig, T. Kostiuk, J. Delgado, R. Schieder, Temperatures in Venus upper atmosphere from mid infrared heterodyne spectroscopy of $\mathrm{CO}_{2}$ around $10 \mathrm{~m}$ wavelength. Planet. Space Sci. 56(10), 1407-1413 (2008). https://doi.org/10.1016/j.pss.2008.05.008

G. Sonnabend, P. Krötz, F. Schmülling, T. Kostiuk, J. Goldstein, M. Sornig, D. Stupar, T. Livengood, T. Hewagama, K. Fast, A. Mahieux, Thermospheric/mesospheric temperatures on Venus: results from ground-based high-resolution spectroscopy of $\mathrm{CO}_{2}$ in 1990/1991 and comparison to results from 2009 and between other techniques. Icarus 217, 856-862 (2012)

L.A. Sromovsky, H.E. Revercomb, V.E. Suomi, Temperature structure in the lower atmosphere of Venus: new results derived from Pioneer Venus entry probe measurements. Icarus 62, 458-493 (1985)

D.O. Staley, The adiabatic lapse rate in the Venus atmosphere. J. Atmos. Sci. 32, 1005-1016 (1970)

P. Stone, The dynamics of the atmosphere of Venus. J. Atmos. Sci. 32, 1005-1016 (1975). https://doi.org/ 10.1175/1520-0469(1975)032<1005:TDOTAO >2.0.CO;2

J. Strong, W.H. Sinton, Radiometric Observations of Venus. Astrophys. J. 131, 470 (1960)

V.E. Suomi, L.A. Sromovsky, H.E. Revercomb, Net radiation in the atmosphere of Venus: measurements and interpretation. J. Geophys. Res. 85(A13), 8200-8218 (1980)

H. Svedhehm, D. Titov, F. Taylor, O. Witasse, Venus Express mission. J. Geophys. Res. 114, E00B33 (2009). https://doi.org/10.1029/2008JE003290

M. Taguchi, T. Fukuhara, T. Imamura, M. Nakamura, N. Iwagami, M. Ueno, M. Suzuki, G.L. Hashimoto, K. Mitsuyama, Longwave infrared camera onboard the Venus climate orbiter. Adv. Space Res. 40, 861-868 (2017). https://doi.org/10.1016/j.asr.2007.05.085

P. Tanga, T. Widemann, B. Sicardy, J.M. Pasachoff, J. Arnaud, L. Comolli, A. Rondi, S. Rondi, P. Sutterlin, Sunlight refraction in the mesosphere of Venus during the transit on June 8th, 2004. Icarus 218(1), 207-219 (2012). https://doi.org/10.1016/j.icarus.2011.12.004

F.W. Taylor, R. Beer, M.T. Chahine, D.J. Diner, L.S. Elson, R.D. Haskins, D.J. McCleese, J.V. Martonchik, P.E. Reichley, S.P. Bradley, J. Delderfield, J.T. Schofield, C.B. Farmer, L. Froidevaux, J. Leung, M.T. Coffey, J.C. Gille, Structure and meteorology of the middle atmosphere of Venus: infrared remote sensing from the Pioneer obiter. J. Geophys. Res. 85(A13), 7963-8006 (1980) 
F.W. Taylor, D.M. Hunten, L.V. Ksanfomaliti, The thermal balance of the middle and upper atmosphere of Venus, in Venus, ed. by D.M. Hunten, L. Colin, T.M. Donahue, V.I. Moroz (The University of Arizona Press, Tucson, 1983), pp. 650-680

F.W. Taylor, D. Crisp, B. Bezard, Near-infrared sounding of the lower atmosphere of Venus, in Venus-II, ed. by S.W. Bougher, D.M. Hunten, R.J. Phillips (The University of Arizona Press, Tucson, 1997), pp. 325-351

S. Tellmann, M. Pätzold, B. Häusler, M.K. Bird, G.L. Tyler, Structure of the Venus neutral atmosphere as observed by the Radio Science experiment VeRa on Venus Express. J. Geophys. Res., Planets 114, E13 (2009)

S. Tellmann, B. Häusler, D.P. Hinson, G.L. Tyler, T.P. Andert, M.K. Bird, T. Imamura, M. Pätzold, S. Remus, Small-scale temperature fluctuations seen by the VeRa Radio Science Experiment on Venus Express. Icarus 221, 471-480 (2012)

D.V. Titov, Radiative balance in the mesosphere of Venus from the Venera-15 infrared spectrometer results. Adv. Space Res. 15, 73-77 (1995)

D.V. Titov, M. Bullock, D. Crisp, N. Renno, F.W. Taylor, L.V. Zasova, Radiation in the atmosphere of Venus, in Exploring Venus as Terrestrial Planet, ed. by L.W. Esposito, E.R. Stofan, Th.E. Cravens. AGU Geophysical Monograph, vol. 176 (2007), pp. 121-138

D.V. Titov, H. Svedhem, F.W. Taylor, S. Barabash, J.-L. Bertaux, P. Drossart, V. Formisano, B. Häusler, O. Korablev, W.J. Markiewicz, D. Nevejans, M. Pätzold, G. Piccioni, J.-A. Sauvaud, T.L. Zhang, O. Witasse, J.-C. Gerard, A. Fedorov, A. Sanchez-Lavega, J. Helbert, R. Hoofs, Venus Express: highlights of the nominal mission. Sol. Syst. Res. 43, 185-209 (2009)

D.V. Titov, W.J. Markiewicz, N.I. Ignatiev, Morphology of the cloud tops as observed by the Venus Express Monitoring Camera. Icarus 217(2), 682-701 (2012). https://doi.org/10.1016/j.icarus.2011.06.020

D.V. Titov, G. Piccioni, P. Drossart, W.J. Markiewicz, Radiative energy balance in the Venus atmosphere, in Towards Understanding the Climate of Venus. ISSI Scientific Report Series, vol. 11 (Springer, New York, 2013), pp. 23-53

D. Titov, N. Ignatiev, K. McGouldrick, V. Wilquet, C. Wilson, Clouds and hazes. Space Sci. Rev. (2018, in press)

M.G. Tomasko, The thermal balance of the lower atmosphere of Venus, in Venus, ed. by D.M. Hunten, L. Colin, T.M. Donahue, V.I. Moroz (University of Arizona Press, Tucson, 1983), pp. 604-631

M.G. Tomasko, L.R. Doose, P.H. Smith, A.P. Odell, Measurements of the flux of sunlight in the atmosphere of Venus. J. Geophys. Res. 85(A13), 8167-8186 (1980a)

M.G. Tomasko, P.H. Smith, V.E. Suomi, L.A. Sromovsky, H.E. Revercomb, F.W. Taylor, D.J. Martonchik, A. Sieff, R. Boese, J.B. Pollack, A.P. Ingersoll, G. Schubert, C.C. Covey, The thermal balance of Venus in light of the Pioneer Venus mission. J. Geophys. Res. 85(A13), 8187-8199 (1980b)

M.G. Tomasko, L.R. Doose, P.H. Smith, The absorption of solar energy and the heating rate in the atmosphere of Venus. Adv. Space Res. 5, 71-79 (1985)

O.B. Toon, J.B. Pollack, R.P. Turco, The ultraviolet absorber on Venus - amorphous sulfur. Icarus 51, 358373 (1982)

L.D. Travis, On the origin of ultraviolet contrasts on Venus. J. Atmos. Sci. 32, 1190-1200 (1975)

H.C. Van de Hulst, Light Scattering by Small Particles (Dover, New York, 1981)

A.C. Vandaele, A. Mahieux, S. Robert, R. Drummond, V. Wilquet, J.L. Bertaux, Carbon monoxide short term variability observed on Venus with SOIR/VEX. Planet. Space Sci. 113, 237-255 (2015)

A.C. Vandaele, O. Korablev, D. Belyaev, S. Chamberlain, D. Evdokimova, Th. Encrenaz, L. Esposito, K.L. Jessup, F. Lefèvre, S. Limaye, A. Mahieux j, E. Marcq, F.P. Mills 1, F. Montmessin, C.D. Parkinson, S. Robert, T. Roman, B. Sandor, A. Stolzenbach, C. Wilson, V. Wilquet, Sulfur dioxide in the Venus atmosphere, II: spatial and temporal variability. Icarus 295, 1-15 (2017). https://doi.org/10.1016/j.icarus.2017.05.001

U. von Zahn, V.I. Moroz, Composition of the Venus atmosphere below $100 \mathrm{~km}$ altitude. Adv. Space Res. 5, 173-195 (1985)

U. von Zahn, S. Kumar, H. Niemann, R.G. Prinn, Composition of the Venus atmosphere, in Venus, ed. by D.M. Hunten, L. Colin, T.M. Donahue, V.I. Moroz (University of Arizona Press, Tucson, 1983), pp. 299430

R.B. Wattson, L.S. Rothman, Direct numerical diagonalization-wave of the future. J. Quant. Spectrosc. Radiat. Transf. 48, 763-780 (1992)

M. Wild, D. Folini, C. Schär, N. Loeb Ellsworth, G. Dutton, G. König-Langlo, The global energy balance from a surface perspective. Clim. Dyn. 40, 3107-3134 (2013). https://doi.org/10.1007/ s00382-012-1569-8

R. Wildt, Note on the surface temperature of Venus. Astrophys. J. 91, 266-268 (1940)

V. Wilquet, R. Drummond, A. Mahieux, S. Robert, A.C. Vandaele, J.L. Bertaux, Optical extinction due to aerosols in the upper haze of Venus: four years of SOIR/VEX observations from 2006 to 2010. Icarus 217, 875-881 (2012) 
J.H. Woodman, E.S. Barker, Relative spectrophotometry of ultraviolet clouds on Venus. Bull. Am. Astron. Soc. 5, 301 (1975)

O.I. Yakovlev, A.I. Efimov, S.S. Matyugov, T.S. Timofeeva, E.V. Chub, G.D. Yakovleva, The nighttime atmosphere of Venus from Venera 9 and 10 radio occultations. Kosm. Issled. 16, 113-119 (1978)

O.I. Yakovlev, S.S. Matyugov, A.I. Efimov, V.N. Gubenko, A.I. Kucheryavenkov, A.S. Vyshlov, G.D. Yakovleva, The atmosphere of Venus in the Northern polar region from radio occultation data obtained by Venera 15 and 16. Kosm. Issled. 25, 275-284 (1987)

A. Yamazaki, M. Yamada, Y.J. Lee, S. Watanabe, T. Horinouchi, S. Murakami, T. Kouyama, K. Ogohara, T. Imamura, T.M. Sato, Y. Yamamoto, T. Fukuhara, H. Ando, K. Sugiyama, S. Takagi, H. Kashimura, S. Ohtsuki, N. Hirata, G.L. Hashimoto, M. Suzuki, C. Hirose, M. Ueno, T. Satoh, T. Abe, N. Ishii, M. Nakamura, Ultraviolet imager on Venus orbiter Akatsuki and its initial results. Earth Planets Space 70(1), 23 (2018). https://doi.org/10.1186/s40623-017-0772-6

R.E. Young, R.L. Walterscheid, G. Schubert, A. Seiff, V.M. Linkin, A.N. Lipatov, Characteristics of gravity waves generated by surface topography on Venus: comparison with the VEGA balloon results. J. Atmos. Sci. 44, 2628-2639 (1987)

L.V. Zasova, V.A. Krasnopolsky, V.I. Moroz, Vertical distribution of $\mathrm{SO}_{2}$ in upper cloud layer of Venus and origin of UV absorption. Adv. Space Res. 1(9), 13-16 (1981). https://doi.org/10.1016/ 0273-1177(81)90213-1

L.V. Zasova, I.A. Khatountsev, V.I. Moroz, N.I. Ignatiev, Structure of the Venus middle atmosphere: Venera 15 Fourier spectrometry data revisited. Adv. Space Res. 23(9), 1559-1568 (1999). https://doi.org/10.1016/ S0273-1177(99)00169-6

L.V. Zasova, V.I. Moroz, V.M. Linkin, I.V. Khatuntsev, B.S. Maiorov, Structure of the Venusian atmosphere from surface up to $100 \mathrm{~km}$. Cosm. Res. 44, 364-383 (2006)

L.V. Zasova, N.I. Ignatiev, I.A. Khatountsev, V.M. Linkin, Structure of the Venus atmosphere. Planet. Space Sci. 55, 1712-1728 (2007a)

L.V. Zasova, V.I. Moroz, V. Formisano, N.I. Ignatiev, I.V. Khatuntsev, Exploration of Venus with the Venera15 IR Fourier spectrometer and the Venus Express planetary Fourier spectrometer. Cosm. Res. 44(4), 349-363 (2007b). https://doi.org/10.1134/S0010952506040083 